\title{
Identification of filamentary structures in the environment of superclusters of galaxies in the Local Universe ${ }^{\star}$
}

\author{
Iris Santiago-Bautista ${ }^{1,2}$, César A. Caretta ${ }^{2}$, Héctor Bravo-Alfaro ${ }^{2}$, Etienne Pointecouteau ${ }^{1}$, and Heinz Andernach ${ }^{2}$ \\ 1 IRAP, Université de Toulouse, CNRS/CNES/UPS, Toulouse, France \\ e-mail: isantiago@irap.omp.eu \\ 2 Departamento de Astronomía, DCNE-CGT, Universidad de Guanajuato, Guanajuato CP 36023, Mexico \\ e-mail: isantiago@astro.ugto.mx
}

Received 28 July 2019 / Accepted 3 Februrary 2020

\begin{abstract}
Context. Characterization of the internal structure of the superclusters of galaxies (walls, filaments, and knots where the clusters are located) is crucial for understanding the formation of the large-scale structure and for outlining the environment where galaxies evolved in the last few gigayears.

Aims. We aim to detect the compact regions of high relative density (clusters and rich groups of galaxies), to map the elongated structures of low relative density (filaments, bridges, and tendrils of galaxies), and to characterize the galaxies that populate the filaments and study the environmental effects they are subject to.

Methods. We used optical galaxies with spectroscopic redshifts from the SDSS-DR13 inside rectangular boxes encompassing the volumes of a sample of 46 superclusters of galaxies up to $z=0.15$. A virial approximation was applied to correct the positions of the galaxies in the redshift space for the "finger of God" projection effect. Our methodology implements different classical pattern recognition and machine-learning techniques (Voronoi tessellation, hierarchical clustering, graph-network theory, and minimum spanning trees, among others), pipelined in the Galaxy System-Finding algorithm and the Galaxy Filament-Finding algorithm.

Results. In total, we detected 2705 galaxy systems (clusters and groups, of which 159 are new) and 144 galaxy filaments in the 46 superclusters of galaxies. The filaments we detected have a density contrast of above 3 , with a mean value of around 10, a radius of about $2.5 \mathrm{~h}_{70}^{-1} \mathrm{Mpc}$, and lengths of between 9 and $130 \mathrm{~h}_{70}^{-1} \mathrm{Mpc}$. Correlations between the galaxy properties (mass, morphology, and activity) and the environment in which they reside (systems, filaments, and the dispersed component) suggest that galaxies closer to the skeleton of the filaments are more massive by up to $25 \%$ compared to those in the dispersed component; $70 \%$ of the galaxies in the filament region present early-type morphologies and the fractions of active galaxies (both AGNs and star-forming galaxies) seem to decrease as galaxies approach the filament.

Conclusions. Our results support the idea that galaxies in filaments are subject to environmental effects leading them to be more massive (probably due to larger rates of both merging and gas accretion), less active both in star formation and nuclear activity, and prone to the density-morphology relation. These results suggest that preprocessing in large-scale filaments could have significant effects on galaxy evolution.
\end{abstract}

Key words. galaxies: groups: general - galaxies: clusters: general - large-scale structure of Universe - methods: data analysis galaxies: evolution

\section{Introduction}

The large-scale structure (LSS) of the Universe is composed of a network of groups and clusters of galaxies, elongated filaments, widely spread sheets, and voids (e.g., Peebles 1980; Davis et al. 1982; Bond et al. 1996). Both the $\Lambda$ CDM cosmological model (e.g., Bond \& Szalay 1983; Doroshkevich \& Khlopov 1984) and recent numerical $N$-body simulations (e.g., Millennium, Springel et al., 2005; Bolshoi, Klypin et al., 2011; Illustris, Vogelsberger et al., 2014) reinforce the idea that these structures are assembled under the effect of gravity generated by the total matter content. Since the baryonic matter follows, to first order, the distribution of the dark matter, the galaxies and gas populate these substructures accordingly (e.g., Eisenstein et al. 2005). Moreover, there is increasing evidence that the galaxy properties (e.g., mass, activity, morphology, luminosity, surface brightness, orientation, etc.) correlate with the LSS environment in

* Full Table 4 is only available at the CDS via anonymous ftp to cdsarc.u-strasbg.fr (130.79.128.5) or via http://cdsarc. u-strasbg.fr/viz-bin/cat/J/A+A/637/A31 which they are located (e.g., Smargon et al. 2012; Scoville et al. 2013; Poudel et al. 2016; Kuutma et al. 2017; Chen et al. 2017; Wang et al. 2018) or, more specifically, with the internal structure of the supercluster (e.g., Einasto et al. 2008; Gallazzi et al. 2009; Gavazzi et al. 2010; Cybulski et al. 2014; Guglielmo et al. 2018). Furthermore, theoretical studies (e.g., Cen \& Ostriker 1999) suggest that from one-half to two-thirds of the baryonic matter in the Universe is hidden in the filamentary structures of the LSS. Therefore, characterization of the LSS (e.g., topology, density, temperature, dynamical state, matter distribution, and its evolution over time) is an important step in placing constraints on the current cosmological models.

Galaxy clusters are well studied through their gas component since they are the densest regions of the LSS. However, the gas in filaments is most likely in a relatively cool $\left(T \sim 10^{5}-10^{7} \mathrm{~K}\right.$, or $0.01-1 \mathrm{keV}$ ) and relative low-density gas phase called the warm hot intergalactic medium (WHIM). There is already some evidence for such gas from X-ray emission observed within pairs of close clusters (e.g., Ursino et al. 2015; Alvarez et al. 2018). In addition, the WHIM between pairs of clusters has 
been observed through the Sunyaev-Zel'dovich effect (SZ; e.g., Planck Collaboration VIII 2013). Tanimura et al. (2019) carried out statistical analyses using Planck SZ observations in the regions of superclusters. The results of these latter authors provide evidence for inter-cluster gas at a temperature of $T \sim$ $8 \times 10^{6} \mathrm{~K}$. Also, Eckert et al. (2017) presented deep X-ray observations of the galaxy cluster Abell 2744, the analysis of which suggests a gas fraction of between 5 and $15 \%$ for the filaments that surround the cluster and a plasma temperature of $1-2 \times 10^{7} \mathrm{~K}$. Therefore, the characterization of these structures through observables like X-ray emission or the SunyaevZel'dovich effect is still challenging due to the low density and temperature of the WHIM.

An alternative is to analyze the galaxy distribution at large scales. Recently, with the availability of large sky area databases such as the Two Degree Field Galaxy Redshift Survey (2dFGRS; Colless et al. 2001), the 2MASS Redshift Survey (2MRS; Huchra et al. 2012), and the Sloan Digital Sky Survey (SDSS; Albareti et al. 2017), the development of accurate structure-detection algorithms has become an even more important concern for astronomy. Visually, the galaxy distribution shows filamentary ridge-like structures that connect massive clusters and groups. However, the identification of these structures through a computational algorithm is not easy to achieve. A good algorithm should first produce an identification that resembles the human visual perception. It should also deliver quantitative results and be founded in a robust and well-defined numerical theory. All of this must be done in an acceptable amount of time with reasonable computational resources.

Currently, there are several filament-finding algorithms that have been tested on the basis of $N$-body simulations. For example, Aragón-Calvo et al. (2007) present the multi-scale morphology filter method (MMF), which divides cosmic structure into nodes (clusters), filaments, and walls using a smoothing over a range of scales (from a Delaunay tessellation reconstruction, DFTE) and a morphological response filter. Another approach, presented by Aragón-Calvo et al. (2010), makes use of a watershed segmentation technique to trace the spines of the filaments. Alternatively, Cautun et al. (2013) propose an algorithm called NEXUS, that takes into account the density, tidal field, velocity divergence, and velocity shear of the galaxies. Other examples are the algorithm by González \& Padilla (2010), which uses the binding energy for selecting the filament members; and the DisPerSE algorithm, by Sousbie (2011), based on the Morse theory - both use Delaunay-Voronoi tessellation based on density estimations.

On the other hand, several attempts have been made to trace the distribution of the real cosmic web using the SDSS database. For example, Sousbie et al. (2008) applied their local skeleton method to samples of Data Release (DR) 4, which allowed them to estimate the mean filament length per unit volume. The algorithm by Bond et al. (2010), called the smoothed Hessian major axis filament finder (SHMAFF), was applied to the SDSS-DR6 after removing the finger-of-God (FoG) effect. Platen et al. (2011) compared three different reconstruction techniques, namely the DFTE, the natural neighbor field estimator (NNFE), and a Kriging interpolation, and searched for voids also in DR6. These latter authors found that DFTE works quantitatively better than the others while the Kriging and NNFE have a better performance in producing visually appealing reconstructions than DFTE. Smith et al. (2012) applied their multi-scale probability mapping (MSPM), which combines probability and scale density information with a friends-of-friends (FoF) algorithm, over the SDSS-DR7 galaxies. This method allowed these latter authors to recover structures from clusters to filaments of up to $\sim 10 \mathrm{~h}^{-1} \mathrm{Mpc}$. Tempel et al. (2014) applied a Bisous model on the SDSS-DR8 spectroscopic galaxies to trace the filament spines. This latter method adjusts cylinders to the galaxy positions applying a stochastic metric. The subspace constrained mean shift (SCMS) approach, which uses a kernel density estimator (KDE), was applied by Chen et al. (2016) to DR7 and by Chen et al. (2015) to DR12. This method allows the identification of high-density regions by smoothing the galaxy distribution. These latter authors applied this technique over slices of 0.05 in redshift for the SDSS sky area. Moreover, Alpaslan et al. (2014) found, for the Galaxy And Mass Assembly (GAMA) survey, that there are fine filaments embedded inside the SDSS voids. These structures, referred to as "tendrils", have a lower density than the SDSS filaments, appear to be morphologically distinct, are more isolated, and span shorter distances. A comprehensive review and comparative analysis of the above algorithms can be found in Libeskind et al. (2018).

Another approach to analyzing the LSS is to study the superclusters of galaxies. These are traditionally defined as concentrations of galaxy clusters (e.g., Abell 1961; Einasto et al. 2001; Chow-Martinez et al. 2014), that build up the cosmic web from a network of connected high-density nodes; or directly from the distribution of galaxies (e.g., Luparello et al. 2011; Costa-Duarte et al. 2011; Liivamägi et al. 2012). They can also be defined kinematically by mapping galaxy peculiar velocity flows, a technique still restricted to the very nearby Universe (Tully et al. 2014; Dupuy et al. 2019). This last method is the closest to a purely gravitational-potential-based approach, and allows the identification of the "basins of attraction" that partition the Universe into cells or cocoons (e.g., Dupuy et al. 2019; Einasto et al. 2019). For this work we adopted the supercluster second-order clustering definition for determining the superclusters of the sample. These systems are not virialized and the contents of the inter-cluster medium (dark matter halos, gas, and galaxies) dynamically interact and organize by falling through the gravitational potential of the more massive structures, forming walls, filaments, groups, and clusters. As shown in Tanaka et al. (2007), the possibility of finding elongated chain-like structures increases in superclusters. Also, following the classification of superclusters by Einasto et al. (2014) into filament-type and spider-type, both have filaments, in a linear or radial configuration, respectively. Following this approach, Cybulski et al. (2014), for example, applied a combination of Voronoi tessellation and minimum spanning tree (MST) techniques over the Coma supercluster region in order to search for bridges between clusters of galaxies.

Motivated by the above context, we developed a methodol$o g y^{1}$ for the identification of structures in the environment of superclusters using the galaxies embedded in them. We restrict our study to the SDSS-DR13 area, and use only galaxies with spectroscopic redshifts for our analysis. The approach we follow seeks to detect structures by using only the geometrical information of the galaxy distribution. Using different patternrecognition methods, we identify high- to moderate-density galaxy systems, and low-density filaments connecting them. This allows the identification of structures over a wide range of scales $(1-100 \mathrm{Mpc})$, from groups to long filaments. Moreover, the identified structures are validated through comparisons with previously reported catalogs. We also carried out a qualitative validation through a kernel method which is one of the most commonly used methodologies for the detection of overdensity

https://gitlab.com/iris.santiagob89/LSS_structures 
regions. Our aim is to investigate whether previous filament candidates in the sample, identified from chains of Abell/ACO clusters, are bona-fide structures and to characterize their galaxy populations. Finally, we studied the relation between the galaxy properties and the supercluster environment in which they reside (e.g., systems, filaments, and the dispersed component of the superclusters).

This paper is organized as follows: in Sect. 2 we present the data for the sample of superclusters under analysis and the sample of galaxies from the SDSS survey. In Sect. 3 we describe in detail the implementation of mathematical tools and patternrecognition methods applied for the detection of high-density regions (clusters and groups) and for the skeletonization of the low-density filamentary structures. In Sect. 4 we describe the algorithm for detecting clusters and groups of galaxies inside supercluster boxes, and in Sect. 5 we present the algorithm for finding the filaments and their skeletons. In Sect. 6 we describe the application of the algorithms to one of the superclusters, MSCC 310, as an example of their use. Section 7 is devoted to the validation and evaluation of the methodology and discussion of its results. In Sects. 8 and 9 we present the results concerning the analyses of the galaxy properties as a function of the supercluster environment. We also discuss these results and compare with previously reported results. Finally, in Sect. 10 we present the conclusions of this work. Throughout this paper we assume the Hubble constant $H_{0}=70 \mathrm{~h}_{70} \mathrm{~km} \mathrm{~s}^{-1} \mathrm{Mpc}^{-1}$, the matter density $\Omega_{m}=0.3$, and the dark-energy density $\Omega_{\Lambda}=0.7$.

\section{The data}

\subsection{The superclusters and filament candidates}

We are interested in unveiling and studying LSS filaments, which can be defined as chains of clusters connected by bridges of galaxies and probably by gas and dark matter. As mentioned previously, these elongated structures should most likely be found in superclusters since they probably just passed the quasi nonlinear regime described by the Zel'dovich's approximation (1970; see also the "sticking model" by Shandarin \& Zel'dovich 1989). In the current evolutionary stage of LSS, superclusters are a network of sheets, filaments, and knots (clusters and groups) of galaxies, gas, and dark matter, just starting a global gravitational collapse process.

We selected a sample of superclusters of galaxies from the Main SuperCluster Catalogue (MSCC; Chow-Martinez et al. 2014) that are inside the SDSS region (in order to have a sample of galaxy data that is as homogeneous as possible). The original MSCC is an all-sky catalog that contains 601 superclusters, identified in a complete sample of rich Abell/ACO clusters with updated redshifts from 0.02 to 0.15 using a tunable FoF algorithm. From these superclusters, 166 are inside the SDSSDR13 region. For this work we selected those superclusters with five or more clusters with a box volume (see below) inside the SDSS-DR13 survey area. In addition, we used the list of filament candidates for MSCC superclusters by Chow-Martínez et al. (in prep.) as a reference in order to select the superclusters with the most promising filaments. Roughly speaking, these filament candidates were identified as chains of at least three clusters - members of the superclusters - separated by less than $20 \mathrm{~h}_{70}^{-1} \mathrm{Mpc}$ from each other. The present work also intends to validate these filament candidates by searching for the bridges of galaxies that we expect to connect them. It is worth mentioning that some of the filament candidates may turn out to be only chance configurations, with no bridges of galaxies connecting the clusters of a chain. Also, some bridges may exist, but not necessarily along the straight lines connecting the clusters. Our final sample consists of 46 superclusters of galaxies, which are listed in Table 1.

For the Abell/ACO clusters and for the galaxies in the supercluster box volumes (see Sect. 2.3), we first transformed their radial-angular coordinates to rectangular coordinates as follows:

$X=D_{\mathrm{C}} \cos (\delta) \cos (\alpha)$,

$Y=D_{\mathrm{C}} \cos (\delta) \sin (\alpha)$,

$Z=D_{\mathrm{C}} \sin (\delta)$,

where $D_{\mathrm{C}}$ is the co-moving distance as obtained using the spectroscopic redshift and the cosmological parameters indicated above.

\subsection{The SDSS galaxies}

The main galaxy sample of SDSS-DR13 (Albareti et al. 2017) is a suitable database to search for filamentary structures on the LSS because (i) it covers a large sky area (14555 square degrees) containing various MSCC superclusters; (ii) it contains homogeneous photometric and spectroscopic data for galaxies with an astrometric precision of 0.1 arcsec rms and uncertainty in radial velocities of about $30 \mathrm{~km} \mathrm{~s}^{-1}$ (Bolton et al. 2012); (iii) it is roughly complete to the magnitude limit of the main galaxy sample $\left(r_{\text {Pet }}=17.77\right)$, which corresponds to an average $z \sim 0.1$, going (inhomogeneously) deeper for data releases after DR7 (Abazajian et al. 2009); and (iv) at the limit of our sample, $z=0.15$, the SDSS spectra are complete for galaxies brighter than $M_{r} \sim-21$.

SDSS-DR7 joins the SDSS-I/II spectra for one million galaxies and quasars. It has $\sim 6 \%$ incompleteness due to fiber collisions (Strauss et al. 2002) and another $\sim 7 \%$ incompleteness attributed to pipeline misclassification (Rines et al. 2007). These spectra are included in the final data release of the SDSS-III (Alam et al. 2015). The Baryon Oscillation Spectroscopic Survey (BOSS) is part of the SDSS-III observations and obtained spectra for another 1.4 million galaxies. The BOSS observations are divided in two main samples, the low-redshift LOWZ $(z<0.4)$ and the high-redshift CMASS $(0.4<z<0.7)$ galaxy samples. The SDSS-DR13 (Albareti et al. 2017) includes spectra for more than 2.6 million galaxies and quasars.

Although photometric redshifts are available for SDSS galaxies, for this work we selected those objects listed on the SpecObj sample for which spectroscopic redshifts are available (downloaded from the SkyServer web service) and that are described as extragalactic (i.e., galaxies and low-z quasars). The SpecObj table contains the best and unique spectra for the same location within 2 arcsec; these are referred to as "sciencePrimary" objects. We considered galaxies within a redshift range from 0.01 to 0.15 and selected spectra with quality flag "good" or "marginal". Because the kind of study presented here relies on the galaxy distance measurement, we restricted our analysis to galaxies for which spectroscopic redshift measurements are available to its higher accuracy. However, galaxies for which photometric redshift is available can be included in the sample in further analyses to test whether or not their addition increases the filament signal of detection.

For the present work we also made use of value-added subproduct catalogs such as the Max Planck for Astrophysics and Johns Hopkins University (MPA-JHU) catalog (Brinchmann et al. 2004; Kauffmann et al. 2003; Tremonti et al. 2004). These latter authors calculated different galaxy 
Table 1. Sample of MSCC superclusters used in the present work.

\begin{tabular}{|c|c|c|c|c|c|c|}
\hline $\begin{array}{l}\mathrm{SCl} \text { ID } \\
\text { (MSCC) } \\
(1)\end{array}$ & Name & $\begin{array}{l}\text { RA, Dec } \\
\text { [deg, deg] } \\
\text { (3) }\end{array}$ & (4) & $N_{\mathrm{Cl}}$ & $N_{\text {fil }}$ & $\begin{array}{l}\text { Abell/ACO } \\
\text { clusters } \\
(7)\end{array}$ \\
\hline 55 & & $17.75,15.44$ & 0.0614 & 5 & 1 & A0150 A0152 A A0154 B A0158 B A0160 B \\
\hline 72 & & $25.17,0.64$ & 0.0802 & 5 & 1 & A0181 A A0208 A A0237 A A0267 B A0279 A \\
\hline 75 & & $28.09,-5.15$ & 0.0937 & 7 & 1 & A0256 A A0256 B A0266 A0269 A0274 A A0274 B A0277 \\
\hline 76 & & $28.35,-2.61$ & 0.1299 & 16 & 3 & $\begin{array}{l}\text { A0211 A0233 e A0255 A0256 C A0261 B A0265 A0267 C A0268 B A0271 A0274 C A0279 B } \\
\text { A0281 A0285 A0295 D A0303 C A0308 e }\end{array}$ \\
\hline 175 & & $125.29,17.07$ & 0.0942 & 6 & 1 & A0635 A A0650 B A0651 A A0657 A A0658 A A0659 \\
\hline 184 & & $130.10,30.24$ & 0.1056 & 6 & 1 & A0671 B A0690 C A0694 A0695 B A0699 B A0705 A \\
\hline 211 & & $147.87,64.88$ & 0.1191 & 8 & 1 & A0764 A0802 A0804 B A0845 A0871 e A0906 e A0975 A1014 A \\
\hline 219 & & $153.99,19.14$ & 0.1155 & 5 & 1 & A0938 B A0942 A A0952 A A0991 B A0994 A \\
\hline 222 & & $155.14,49.21$ & 0.1382 & 10 & 2 & A0915 B A0927 A A0950 A A0965 A A0990 A1002 A A1003 C A1003 D A1004 A1040 C \\
\hline 223 & & $155.24,62.94$ & 0.1399 & 5 & 1 & A0917 A0947 A A0962 A A1025 A A1025 B \\
\hline 229 & & $156.14,33.03$ & 0.1423 & 7 & 2 & A0924 A0951 A0982 A1007 B A1036 A1045 A1053 B \\
\hline 236 & & $156.76,10.38$ & 0.0328 & 6 & 1 & A0938 A A0957 A A0999 A A1016 A A1020 A A1142 A \\
\hline 238 & & $156.98,39.55$ & 0.1068 & 21 & 4 & $\begin{array}{lllllll}\text { A0967 A A0971 A A0971 B A0972 A } & \text { A0995 A } & \text { A0997 A } & \text { A0997 B } & \text { A0997C A1010 B } \\
\text { A1021 B A1021 C A1021 D A1026 } & & & & & \end{array}$ \\
\hline & & & & & & B A1028 A A1031 A A1031 B A1033 A1040 A A1050 A A1054 A A1055 \\
\hline 248 & & $159.49,44.26$ & 0.1246 & 5 & 1 & A1040 B A1050 B A1054 B A1056 A1074 A \\
\hline 264 & & $165.29,12.20$ & 0.1161 & 8 & 1 & A1105 C A1116 A A1129 A A1141 A A1147 A A1157 A1201 B A1209 A \\
\hline 266 & & $165.91,11.85$ & 0.1273 & 8 & 1 & A1131 A1137 B A1141 B A1147 B A1152 A1159 A1183 A A1209 B \\
\hline 272 & & $167.83,41.33$ & 0.0760 & 6 & 1 & A1173 A1174 A A1187 A1190 A1193 A A1203 \\
\hline 277 & & $169.41,49.67$ & 0.1103 & 7 & 1 & A1154 A1202 B A1218 B A1222 A1225 A1227 A A1231 A \\
\hline 278 & Leo & $169.37,28.46$ & 0.0333 & 6 & 1 & A1177 B A1179 B A1185 A A1228 A A1257 A A1267 A \\
\hline 283 & & $170.79,20.34$ & 0.1379 & 12 & 3 & A1177 C A1188 A1230 B A1232 B A1242 A A1243 B A1247 e A1251 A1268 A1272 A1274 A1278 \\
\hline 295 & Coma & $173.63,23.11$ & 0.0223 & 5 & 1 & A1100 A A1177 A A1179 A A1367 A1656 \\
\hline 310 & UMa & $175.91,55.23$ & 0.0639 & 21 & 3 & $\begin{array}{l}\text { A1212 A1270 A1291 A A1291 B A1291 C A1318 A A1318 B A1324 A A1324 B A1349 A A1349B } \\
\text { A1377 A1383 A1396 A A1396 B A1400 A A1400 B A1400 C A1436 A1452 A1457 A }\end{array}$ \\
\hline 311 & & $176.12,9.93$ & 0.0833 & 8 & 1 & A1337 A A1342 A A1358 A A1362 B A1372 A A1379 A1385 A A1390 \\
\hline 314 & & $177.07,-2.01$ & 0.0788 & 6 & 1 & A1364 A A1376 A A1386 A A1389 A A1399 A A1404 A \\
\hline 317 & & $177.42,-1.59$ & 0.1278 & 13 & 2 & $\begin{array}{l}\text { A1373 A A1373 B A1376C A1386 D A1386E A1386F A1389C A1389 D A1392 A1399C A1407 } \\
\text { A1411 A1419B }\end{array}$ \\
\hline 323 & & $179.66,27.26$ & 0.1396 & 12 & 1 & $\begin{array}{l}\text { A1384 A A1403 A A1403 B A1413 B A1420 C A1425 B A1431 B A1433 C A1444 C A1449 B } \\
\text { A1455 C A1495 }\end{array}$ \\
\hline 333 & & $181.43,29.34$ & 0.0813 & 9 & 1 & A1423 A A1427 A1431 A A1433 A A1444 B A1449 A A1455 B A1515 A A1549 A \\
\hline 335 & & $182.42,29.50$ & 0.0732 & 6 & 1 & A1444 A A1455 A A1478 A A1480 B A1486 A A1519 A \\
\hline 343 & & $183.88,14.31$ & 0.0809 & 5 & 1 & A1474 A1481 A A1499 A A1526 C A1527 A \\
\hline 360 & Dra & $190.94,64.41$ & 0.1055 & 11 & 1 & A1518 A A1539 A A1544 A A1559 A1566 A1579 A A1621 A1640 A A1646 A1674 A A1718 A \\
\hline 386 & & $199.50,38.33$ & 0.0715 & 5 & 1 & A1680 A A1691 A1715 A A1723 B A1749 B \\
\hline 407 & & $208.55,26.70$ & 0.1364 & 6 & 1 & A1797 B A1817 C A1817 e A1818 C A1819 A1824 \\
\hline 414 & Boo & $211.31,27.32$ & 0.0709 & 24 & 3 & $\begin{array}{l}\text { A1775 A A1775B A1781 B A1795 A1797 A A1800 A1817 A A1818 A A1831 A A1831 B A1832 A } \\
\text { A1863 A A1869 A A1869 B A1873 B A1873C A1874 A A1886 A A1898 A A1903 A A1908 A } \\
\text { A1909 A A1912B A1921 A }\end{array}$ \\
\hline 419 & & $212.33,7.17$ & 0.1122 & 5 & 1 & A1850 A1862 A1866 A A1870 A1881 \\
\hline 422 & & $213.21,28.95$ & 0.1430 & 9 & 2 & A1832 B A1840 B A1854 A1867 A A1874 B A1891 B A1903C A1908 B A1912E \\
\hline 430 & & $216.72,25.64$ & 0.0982 & 6 & 1 & A1909 B A1910 A A1912 A A1912C A1926 A A1927 \\
\hline 440 & BooA & $223.17,22.28$ & 0.1170 & 9 & 1 & A1939 B A1972 A1976 A1980 A1986 A1988 B A2001 A A2006 A2021 C \\
\hline 441 & & $223.22,28.40$ & 0.1249 & 5 & 1 & A1973 A A1982 D A1984 A1990 A A2005 B \\
\hline 454 & & $228.28,7.33$ & 0.0456 & 6 & 1 & A2020 A A2028 A A2033 B A2040 B A2055 A A2063 B \\
\hline 457 & & $228.59,6.98$ & 0.0789 & 6 & 1 & A2028 B A2029 A2033 C A2040 C A2055 B A2063 C \\
\hline 460 & & $229.70,31.17$ & 0.1142 & 9 & 1 & A2025 D A2034 A A2049 A A2056 C A2059 B A2062 A2067 B A2069 A2083 B \\
\hline 463 & $\mathrm{CrB}$ & $232.18,30.42$ & 0.0736 & 14 & 2 & $\begin{array}{l}\text { A2056 A A2056 B A2059 A A2061 A A2065 A2067 A A2073 A A2079 A A2079 B A2089 A2092 A } \\
\text { A2106 A A2122 A A2124 }\end{array}$ \\
\hline 474 & Her & $241.56,16.22$ & 0.0363 & 5 & 2 & A2147 A2151 A2152 A A2153 A A2159 A \\
\hline 484 & & $245.57,42.39$ & 0.1364 & 7 & 1 & A2158 B A2172 A2179 A2183 A2196 A2198 D A2211 A \\
\hline 579 & & $351.82,14.79$ & 0.0427 & 5 & 1 & A2572 A2589 A2593 A A2593 B A2657 \\
\hline 586 & & $354.20,23.67$ & 0.1274 & 5 & 1 & A2611 e A2619 B A2627 A2647e A2650e \\
\hline
\end{tabular}

Notes. The superclusters have five or more Abell cluster members, with $z \leq 0.15$, and inside the SDSS-DR13 region. The columns in the table are as follows: (1) the identify (ID) of the supercluster in MSCC; (2) supercluster proper name, if available; (3) sky coordinates, RA $(\alpha)$ and Dec $(\delta)$, of the supercluster mean position; (4) supercluster mean redshift; (5) supercluster richness (number of member clusters); (6) number of filament candidates found previously in each supercluster; (7) IDs of the Abell/ACO member clusters. 
properties (stellar mass, metallicity, activity type classification, star formation rate, etc.) using the spectra from the SDSS-DR8 galaxies (Aihara et al. 2011). As explained by Tremonti et al. (2004), the galaxy properties in the MPA-JHU catalog are calculated by processing the galaxy spectrum in such a way that even the weaker emission lines are detectable. In order to analyze the morphological distribution of the galaxies in the different supercluster environments we employed the morphological classification provided by Huertas-Company et al. (2011). These latter authors calculate a probabilistic morphological classification for the SDSS-DR7 spectroscopic galaxies by applying deep-learning techniques that make use of their photometry. They also compare their automated classification with a sample of the Galaxy Zoo (Lintott et al. 2008, 2011) visual classification and show that their classification into early and late types is in good agreement with the visual classification.

\subsection{The supercluster boxes}

For each supercluster in Table 1 we selected all the SDSS galaxies (according to the above criteria) located inside the corresponding box volume. These boxes were defined in rectangular coordinates in a way that their walls were set at a distance of $20 \mathrm{~h}_{70}^{-1} \mathrm{Mpc}$ beyond the center of the farthest clusters in each direction, for each supercluster. This extension was applied in order to guarantee that any connection of the supercluster with external structures could be detected. The box volumes of the superclusters vary from $\left(45 \mathrm{~h}_{70}^{-1} \mathrm{Mpc}\right)^{3}$ to $\left(157 \mathrm{~h}_{70}^{-1} \mathrm{Mpc}\right)^{3}$. Compared to the typical sizes of the observed and simulated basins of attraction in Dupuy et al. (2019) $\left[\left(50-100 \mathrm{~h}_{70}^{-1} \mathrm{Mpc}\right)^{3}\right]$, the boxes we use here are slightly larger, as expected, implying that we are sampling the LSS in a general way, and are not restricting the analysis to the densest parts of the superclusters. Our sampling of the superclusters may be compared to that by Krause et al. (2013), that is, broader than the sampling done by, for example, Kopylova \& Kopylov (2006) and Liivamägi et al. (2012).

The properties of the supercluster boxes along with information about the detected galaxy systems (to be described below) are presented in Table 2. In particular, the superclusters MSCC 236, MSCC 314, and MSCC 317 lie close to the limits of the SDSS region: although all their member clusters are inside, their boxes were reduced to a margin of $10 \mathrm{~h}_{70}^{-1} \mathrm{Mpc}$ in only one direction.

Figure 1 shows the diminution of mean volume density of the boxes with redshift due to Malmquist bias. The fitted function will be used as the selection function for the SDSS galaxies considered in this work. It may be noted that the mean densities of the superclusters MSCC 55 and MSCC 579 lie far below the fit. This is also due to the positions of these superclusters close to the border of SDSS coverage: their samplings seem sparse and irregular. In fact, for MSCC 579 one can clearly see the shape of the cones of observation through the galaxy distribution. For this reason, the analysis of these superclusters and of the three cited above must be taken with caution.

It is worth noting that, since we have only the radial velocity component available (redshift), the transformation from radialangular coordinates to rectangular coordinates is more complicated for the galaxies. Their peculiar velocity may bias their redshift-space coordinate, especially when they are members of clusters and groups of galaxies that are subject to the FoG effect. Therefore, for the galaxy data used to detect the filaments, we first applied a correction, as described below, which redefined their individual $D_{\mathrm{C}}$ in Eqs. (1)-(3).

\section{Mathematical tools}

In what follows, we consider the $N$ galaxies in each supercluster volume as a set of points $x_{1}, x_{2}, \ldots, x_{N} \in X$, all part of a sample $X$.

\subsection{Voronoi tessellation}

The Voronoi tessellation (VT; Voronoi 1908) of a sample $X$, $\operatorname{Vor}(X)$, can be defined as the subdivision of a $2 \mathrm{D}$ plane or a 3D space into cells with the property that the seed point $x_{i} \in X$ is located in the cell $v_{i}$ if and only if the Euclidean distance $D_{\mathrm{E}}\left(x_{i}, v_{i}\right)<D_{\mathrm{E}}\left(x_{i}, v_{j}\right)$ for each $v_{j} \in X$ with $j \neq i$. In other words, the VT divides the space into polygonal cells centered on the seed points (in our case, galaxies) in a way that the cell walls are equidistant to all nearest seeds (e.g., Platen et al. 2011). Therefore, the density at each galaxy position $x_{i}$ is determined as $d_{i}=1 / v_{i}$, with $v_{i}$ being the volume (or area) of the cell enclosing the object $x_{i}$. Scoville et al. (2013) and Darvish et al. (2015), for example, use VT to find the high-density regions in sky slices while Cybulski et al. (2014) apply VT to identify the filamentary structures in the Coma cluster region.

\subsection{Hierarchical cluster analysis}

Hierarchical clustering (HC) is a machine-learning method whose objective is to group objects with similar properties. It has been used in several different fields such as artificial intelligence, biology, medicine, and business. In general, it can be used to carry out pattern-recognition analysis, allowing the user to regroup, segment, and classify any kind of data. This method is equivalent to a reduction of the dimensionality of the data and reduces the computing time considerably. In astronomy, the most popular application of $\mathrm{HC}$ has been in the detection of substructures inside galaxy clusters following the algorithm developed by Serna \& Gerbal (1996). This algorithm considers the positions, redshifts, and potential binding energy between pairs of galaxies to detect substructures (see also Guennou et al. 2014).

As we are interested in finding galaxy structures on scales larger than the ones for substructures and for structures that may be less strongly gravitationally bound, we chose to use an agglomerative hierarchical clustering analysis method that considers only the positions and redshifts or 3D estimated positions of galaxies. A detailed description of the HC algorithm can be found in Theodoridis \& Koutroumbas (2009), Theodoridis et al. (2010) and Murtagh \& Contreras (2011). For our analysis we chose Ward's minimum variance clusterization criteria, described in detail by Murtagh \& Legendre (2014). In general, Ward's method works by merging the groups following the criterion:

$\Delta D\left(c_{1}, c_{2}\right)=\frac{\left|c_{1}\right|\left|c_{2}\right|}{\left|c_{1}\right|+\left|c_{2}\right|}\left\|c_{1}-c_{2}\right\|^{2}$

where $\Delta D$ is a term that measures the distance between two groups $c_{1}$ and $c_{2}$, respectively.

In our case, each point is initially considered as a group, subcluster, or singleton, and then each group can be agglomerated with a neighbor that has the minimum $\Delta D$ distance. The agglomeration continues until all points are grouped together.

The results of the $\mathrm{HC}$ clusterization can be represented by a dendrogram or hierarchical tree. A dendrogram represents, in a graphical form, the connections between elements and groups at different levels of agglomeration. The height of each connection line in the tree corresponds to the distance between two connected elements or centroids. This representation also allows 
Table 2. Properties of the supercluster boxes and of the galaxy systems detected inside them using the GSyF algorithm.

\begin{tabular}{|c|c|c|c|c|c|c|c|c|c|c|c|c|}
\hline \multirow{2}{*}{$\begin{array}{l}\mathrm{SCl} \text { ID } \\
(\mathrm{MSCC}) \\
(1)\end{array}$} & \multirow{2}{*}{$\begin{array}{c}V \\
{\left[10^{3} \mathrm{~h}_{70}^{-3} \mathrm{Mpc}^{3}\right]} \\
(2)\end{array}$} & \multirow{2}{*}{$\begin{array}{c}N \\
\text { (DR13) } \\
(3) \\
\end{array}$} & \multirow{2}{*}{$\begin{array}{c}d=N / V \\
{\left[\mathrm{~h}_{70}^{3} \mathrm{Mpc}^{-3}\right]} \\
(4)\end{array}$} & \multirow{2}{*}{$\begin{array}{r}d_{\text {surf }}=N / A \\
{\left[\mathrm{deg}^{-2}\right]} \\
(5) \\
\end{array}$} & \multirow{2}{*}{ 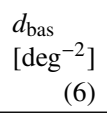 } & \multirow{2}{*}{$\begin{array}{c}N_{\mathrm{gal}} \\
d_{i}>d_{\mathrm{bas}} \\
(7)\end{array}$} & \multirow{2}{*}{$\begin{array}{l}f \\
(8) \\
\end{array}$} & \multirow{2}{*}{$\begin{array}{c}N_{\mathrm{HC}} \\
N_{j} \geq 3 \\
(9) \\
\end{array}$} & \multicolumn{2}{|c|}{$N_{\mathrm{FoG}}$} & \multirow{2}{*}{$\begin{array}{c}R_{\mathrm{vir}} \\
{\left[\mathrm{h}_{70}^{-1} \mathrm{Mpc}\right]} \\
(12) \\
\end{array}$} & \multirow{2}{*}{$\begin{array}{c}\sigma_{v} \\
{\left[\mathrm{~km} \mathrm{~s}^{-1}\right]} \\
(13)\end{array}$} \\
\hline & & & & & & & & & $\begin{array}{c}N_{\text {mem }}<10 \\
(10)\end{array}$ & $\begin{array}{c}N_{\text {mem }} \geq 10 \\
(11)\end{array}$ & & \\
\hline 55 & 424.3 & 812 & 0.0019 & 77.6 & 8.3 & 468 & 27 & 57 & 11 & 5 & $1.1-2.4$ & $245-806$ \\
\hline 72 & 549.7 & 1941 & 0.0035 & 232.0 & 22.1 & 1341 & 18 & 228 & 30 & 22 & $0.9-2.4$ & $184-689$ \\
\hline 75 & 854.6 & 1607 & 0.0019 & 95.8 & 22.6 & 877 & 15 & 69 & 9 & 7 & $1.5-3.4$ & $335-1144$ \\
\hline 76 & 2628.3 & 2617 & 0.0010 & 110.1 & 26.6 & 1536 & 27 & 204 & 14 & 11 & $1.3-3.6$ & $214-1051$ \\
\hline 175 & 577.4 & 2504 & 0.0043 & 116.3 & 25.8 & 1315 & 6 & 172 & 22 & 10 & $1.2-2.7$ & $220-735$ \\
\hline 184 & 692.9 & 2101 & 0.0030 & 70.5 & 20.7 & 1003 & 3 & 137 & 10 & 9 & $1.4-2.7$ & $278-739$ \\
\hline 211 & 814.7 & 1484 & 0.0018 & 28.6 & 11.5 & 654 & 6 & 53 & 2 & 3 & $1.9-2.1$ & $406-496$ \\
\hline 219 & 628.4 & 1913 & 0.0030 & 118.2 & 19.5 & 1273 & 6 & 175 & 13 & 10 & $1.4-3.7$ & $292-1151$ \\
\hline 222 & 955.9 & 1865 & 0.0020 & 97.3 & 20.3 & 885 & 3 & 123 & 10 & 8 & $1.3-4.1$ & $245-1207$ \\
\hline 223 & 777.0 & 776 & 0.0010 & 302.7 & 13.8 & 247 & 3 & 45 & 1 & 3 & $2.1-2.1$ & $475-475$ \\
\hline 229 & 1352.4 & 1855 & 0.0014 & 45.2 & 22.3 & 745 & 3 & 106 & 6 & 2 & $2.2-2.2$ & $498-498$ \\
\hline 236 & 643.6 & 8636 & 0.0134 & 52.9 & 10.3 & 4733 & 3 & 309 & 93 & 73 & $0.5-1.8$ & $105-703$ \\
\hline 238 & 3861.8 & 8328 & 0.0022 & 74.4 & 20.3 & 4860 & 6 & 832 & 38 & 74 & $0.7-4.0$ & $112-1293$ \\
\hline 248 & 690.1 & 1263 & 0.0018 & 72.4 & 17.4 & 564 & 3 & 75 & 5 & 2 & $1.7-2.8$ & $366-730$ \\
\hline 264 & 923.7 & 1704 & 0.0018 & 59.6 & 24.5 & 626 & 3 & 105 & 11 & 12 & $1.3-3.5$ & $245-1026$ \\
\hline 266 & 458.5 & 958 & 0.0021 & 49.5 & 28.8 & 318 & 3 & 55 & 6 & 3 & $1.6-3.1$ & $320-823$ \\
\hline 272 & 138.4 & 1379 & 0.0100 & 135.3 & 28.0 & 654 & 3 & 87 & 10 & 5 & $1.1-2.4$ & 219-699 \\
\hline 277 & 905.3 & 2748 & 0.0030 & 76.0 & 20.2 & 1329 & 6 & 179 & 017 & 9 & $1.4-2.5$ & $278-675$ \\
\hline 278 & 459.3 & 7920 & 0.0172 & 52.3 & 10.3 & 4116 & 6 & 222 & 80 & 35 & $0.5-1.9$ & $112-711$ \\
\hline 283 & 1478.8 & 2320 & 0.0016 & 70.3 & 20.2 & 1379 & 12 & 239 & 10 & 17 & $1.5-3.4$ & 295-907 \\
\hline 295 & 535.5 & 14308 & 0.0267 & 48.5 & 7.2 & 7422 & 6 & 272 & 114 & 46 & $0.4-2.0$ & 74-909 \\
\hline 310 & 1558.8 & 12286 & 0.0079 & 76.9 & 15.7 & 7529 & 6 & 1015 & 116 & 139 & $0.8-3.0$ & $140-1182$ \\
\hline 311 & 958.8 & 5270 & 0.0055 & 91.8 & 22.4 & 3050 & 6 & 416 & 48 & 40 & $0.8-2.4$ & 131-704 \\
\hline 314 & 91.9 & 558 & 0.0061 & 135.2 & 27.0 & 289 & 3 & 49 & 10 & 4 & $1.2-2.3$ & $254-659$ \\
\hline 317 & 438.6 & 840 & 0.0019 & 104.2 & 38.2 & 433 & 6 & 76 & 10 & 5 & $1.8-3.3$ & $366-929$ \\
\hline 323 & 1909.6 & 3330 & 0.0017 & 77.3 & 21.7 & 1764 & 6 & 304 & 17 & 21 & $1.5-3.7$ & 295-1069 \\
\hline 333 & 445.5 & 1968 & 0.0044 & 65.1 & 22.6 & 793 & 3 & 135 & 14 & 27 & $1.1-2.9$ & 221-949 \\
\hline 335 & 574.5 & 3099 & 0.0054 & 62.2 & 21.4 & 1285 & 3 & 211 & 29 & 38 & $0.8-3.0$ & $144-973$ \\
\hline 343 & 427.9 & 2679 & 0.0063 & 105.8 & 19.2 & 1526 & 6 & 196 & 23 & 25 & $0.8-2.4$ & $131-675$ \\
\hline 360 & 657.7 & 2199 & 0.0033 & 80.1 & 15.3 & 934 & 12 & 160 & 15 & 16 & $1.3-2.5$ & $253-653$ \\
\hline 386 & 535.9 & 3256 & 0.0061 & 54.9 & 17.2 & 1600 & 9 & 257 & 33 & 40 & $1.0-2.7$ & $211-852$ \\
\hline 407 & 800.0 & 1126 & 0.0014 & 48.9 & 22.8 & 481 & 12 & 79 & 5 & 5 & $1.5-4.0$ & $280-1184$ \\
\hline 414 & 1245.9 & 10902 & 0.0088 & 93.0 & 23.1 & 6366 & 6 & 1066 & 144 & 161 & $0.8-3.2$ & $140-1191$ \\
\hline 419 & 497.6 & 1723 & 0.0035 & 91.7 & 19.7 & 1103 & 6 & 196 & 25 & 20 & $1.2-3.3$ & 211-976 \\
\hline 422 & 884.6 & 1065 & 0.0012 & 41.9 & 24.2 & 382 & 3 & 62 & 2 & 6 & $2.1-2.3$ & $474-526$ \\
\hline 430 & 437.0 & 1603 & 0.0037 & 88.6 & 22.9 & 871 & 3 & 121 & 20 & 9 & $1.3-2.4$ & $281-647$ \\
\hline 440 & 1017.1 & 3442 & 0.0034 & 99.4 & 72.9 & 917 & 6 & 143 & 24 & 14 & $1.5-3.3$ & $309-935$ \\
\hline 441 & 516.1 & 1058 & 0.0021 & 60.8 & 20.9 & 425 & 6 & 59 & 2 & 3 & $3.0-3.0$ & 796-796 \\
\hline 454 & 389.0 & 5704 & 0.0147 & 99.7 & 18.9 & 3231 & 6 & 524 & 84 & 106 & $0.7-1.9$ & $142-610$ \\
\hline 457 & 529.6 & 4072 & 0.0077 & 129.0 & 22.7 & 2605 & 6 & 443 & 58 & 44 & $1.0-3.1$ & $187-1038$ \\
\hline 460 & 1041.1 & 3499 & 0.0034 & 108.4 & 27.3 & 1925 & 3 & 335 & 35 & 23 & $1.3-3.6$ & $238-1073$ \\
\hline 463 & 959.2 & 8466 & 0.0088 & 121.6 & 22.4 & 5278 & 3 & 898 & 113 & 113 & $0.6-3.1$ & $103-1077$ \\
\hline 474 & 343.8 & 7424 & 0.0216 & 109.2 & 15.3 & 4506 & 9 & 166 & 64 & 26 & $0.6-2.6$ & $122-1115$ \\
\hline 484 & 805.0 & 1319 & 0.0016 & 43.1 & 19.2 & 571 & 6 & 86 & 5 & 4 & $2.3-3.0$ & 536-793 \\
\hline 579 & 658.9 & 1477 & 0.0022 & 142.9 & 1.2 & 1234 & 3 & 149 & 19 & 23 & $0.6-1.9$ & $128-659$ \\
\hline 586 & 962.1 & 373 & 0.0004 & 18.3 & 13.6 & 72 & 3 & 8 & 0 & 0 & -99.0 to -99.0 & -99 to -99 \\
\hline
\end{tabular}

Notes. The value -99 is set when GSyF does not detect systems. The columns in the table are as follows: (1) ID of the supercluster in MSCC; (2) supercluster box volume; (3) number of galaxies inside this volume; (4) mean volume number density inside the box; (5) mean surface (sky projected) number density of the box; (6) baseline density of the box; (7) number of galaxies with surface density above the baseline density; (8) used segmentation parameter $f$; (9) number of HC groups (see Sect. 3.2 for details); (10) remaining groups, after FoG correction, with richness between 5 and 9 galaxies; (11) remaining groups, after FoG correction, with richness higher or equal to 10 galaxies; (12) range of radii for the $N_{\text {mem }} \geq 10$ FoG groups; (13) range of velocity dispersions for the $N_{\text {mem }} \geq 10$ FoG groups.

visualization of the principal branch structures where the singletons are the final leaves. The number of desired groups, $N_{\text {cut }}$, is therefore obtained by cutting the hierarchical tree at a certain level. The exact value of this level depends on the characteristics of the sample or, more precisely, on the underlying physics used to define the groups. Each created group can be represented by a 2D or 3D Gaussian model, $P_{j}(x)$. This allows the groups to be classified by their Gaussian properties, for example by their centroid (mean position, $C_{j}$ ), richness (number of members, $N_{j}$ ), and compactness (covariance, $\sigma_{j}$ ).

\subsection{Graphs}

Graph theory-based algorithms have shown to be a suitable tool to analyze complex networks. Some of the most common subjects where these algorithms are successfully applied are social 


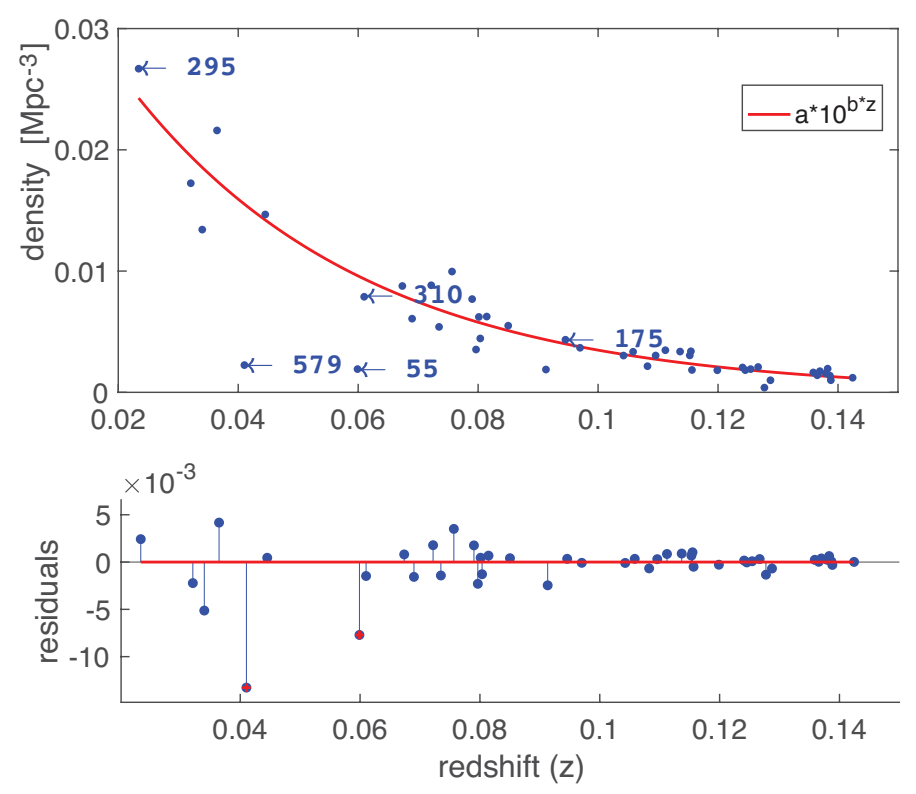

Fig. 1. Distribution of mean volume densities (see fourth column of Table 2) for the 46 superclusters in our sample as function of redshift (blue points). The red line corresponds to the best fit of a powerlaw function. Residuals of the fitting are shown in the bottom panel. MSCC 579 and MSCC 55 were excluded from the fitting.

networks, computer vision, statistics, business, and transportation networks.

A graph is a representation of the connections in a network. It is composed of "nodes" and "edges", where each node represents an object, and the edges represent the connections between nodes. Also, the edges can have weights that represent the strength of the connection. An undirected graph has edges that do not have direction. We can define an undirected graph as $G=(U, E, W)$, with $n$ nodes (or vertices) $u_{i} \in U, m$ edges $e_{k l} \in E$, and a weight set $W$ with a $w_{k l}$ for each edge $e_{k l}$. The information of a graph can be represented by a square adjacency matrix. The values of the matrix entries indicate the weight of the connection between nodes. Hence, the adjacency matrix $A$ of the graph $G$ is defined as:

$[A]_{k l}= \begin{cases}1 & \text { if }\left(u_{k}, u_{l}\right) \in E \\ 0 & \text { otherwise, }\end{cases}$

where $u_{k}$ and $u_{l}$ are nodes in G. One can refer to Ueda \& Itoh (1997) for a discussion on the use of the graph theory approach for quantifying the LSS of the Universe.

\subsection{Minimum spanning tree}

A spanning tree connects all nodes in a graph in a way that does not produce cycles. A graph can contain several unconnected spanning trees. Since the edges in a graph can have weights, the MST algorithm (Graham \& Hell 1985) searches for a spanning tree that minimizes the total weight. This algorithm traces a treelike continuous path for a group of edges and nodes in an optimal way. In particular, the Kruskal MST algorithm analyzes the edges in sequence, sorting them by weight. At the beginning, the shortest edge is analyzed and this would be the first tree branch. The nodes are then added to the tree under three conditions: (i) only one node is added to the tree; (ii) a node is added based on the number of connected edges; (iii) their edges cannot be connected to another existing node in the tree. The process continues with the following edges in the graph until all connected edges are analyzed. Finally, the tree is extracted from the graph and the process begins again with the remaining nodes until all are tested. As its name suggests, the result is a forest of optimized independent trees.

\subsection{Dijkstra's shortest path}

Dijkstra's algorithm (Dijkstra 1959) is a classical method for finding the shortest path between two nodes in a graph. We define a path of length $e_{k l}$ between two nodes $u_{k}$ and $u_{l}$ as a sequence of connected nodes $u_{1}, u_{2}, \ldots, u_{n}$ if $k \neq l \forall k, l \in$ $1, \ldots, n$. In general, Dijkstra's algorithm works as follows. First, an origin is selected by taking the node at the beginning of the path, $u_{0}$. A distance value is then assigned to all nodes: set as zero for the origin, $s\left(u_{0}\right)$, and as infinity for all the other nodes, $s\left(u_{i}\right)=$ inf. Next, all nodes are marked as unvisited and $u_{0}$ is marked as current $a$. The algorithm then calculates the distance from the current node $a$ to all the unvisited nodes connected by the edges $e_{i}$ as $s_{\text {new }}=s\left(e_{a i}\right)+w_{a i}$; here $s\left(e_{a i}\right)$ is the distance from $a$ to the node $u_{i}$ and $w_{a i}$ is the weight of the edge $e_{i}$. If $s\left(e_{a i}\right)+w_{a i}<s\left(e_{i}\right)$, then the distance is updated and the connected node label is updated as the current $a$. After visiting all neighbors of the current node, they are marked as visited. A visited node will not be checked again; the recorded distance $s\left(e_{a i}\right)$ is therefore final and minimal. Finally, if all nodes have been visited, the algorithm stops. Otherwise, the algorithm sets the unvisited nodes with the smallest distance (from the initial node $u_{0}$, considering all nodes in the graph) as the next "current node" and continues from the second step. A detailed description of the algorithm can be consulted in Santanu (2014).

\subsection{Kernel density estimator}

As mentioned before, VT is used to measure the local density at each point position. However, in some cases, it fails to identify large overdensity regions, as mentioned by Cybulski et al. (2014). An alternative to the VT method is to apply KDEs. In general, KDE methods work by adjusting a kernel function over each observation in the sample. However, the choice of the optimal kernel model and its intrinsic parameters is still under investigation in the pattern-recognition community. Also, there have been several attempts to apply adaptive Gaussian model kernels, in other words, to change the size of the Gaussian model as a function of different parameters, such as for example the distance to the nearest neighbor (Chen et al. 2016) or a weighting function (Darvish et al. 2015).

For this work we used the results from the VT method (see Sect. 3.1) as the input parameters for the KDE. We start by fitting an ellipsoid inside each VT cell. Thus, instead of choosing a fixed bandwidth for the kernel, we employ the eigenvalues and eigenvectors of the ellipsoids to calculate a Gaussian kernel $\phi_{\boldsymbol{\Sigma}}$ centered at $\mu$ with covariance matrix $\boldsymbol{\Sigma}$ for each observation. Therefore, each n-dimensional kernel is represented as:

$\phi_{\boldsymbol{\Sigma}}(\mathbf{x}-\mu)=(2 \pi)^{d / 2}|\mathbf{\Sigma}|^{-1 / 2} e^{-1 / 2(\mathbf{x}-\mu)^{T} \boldsymbol{\Sigma}^{-1}(\mathbf{x}-\mu)}$.

The KDE can then be estimated as:

$\hat{p}_{\mathrm{KDE}}(\mathbf{x})=\sum_{i=1}^{N} \alpha_{i} \phi_{\mathbf{\Sigma}_{i}}\left(\mathbf{x}-\mathbf{x}_{i}\right)$,

where $\alpha_{i}$ is a weight factor calculated from the VT cell volume $\left(v_{i}\right)$ as $1 / v_{i}$. 
The identification of the overdensity regions is done through the projection of KDE kernels in 2D planes superposing a regular rectangular grid to the data. Thus, the density estimation is obtained at a given grid intersection by calculating the average density of all kernels that overlap at that point. Observations closer to an evaluating point will therefore contribute more to the density estimation than points farther away from it. Consequently, the density will be higher in areas with many observations than in areas with few observations.

\subsection{Transversal profiles}

The distribution of galaxy properties in filaments is analyzed by constructing transversal profiles. These profiles are calculated by setting up a series of concentric cylinders with axes orientated along the filament skeletons. A bin is then considered to be the volume within two concentric cylinders of radius $R_{\mathrm{cy}}$ and $R_{\mathrm{cy}}+$ $\Delta R_{\text {cy }}$. The occurrence of a galaxy proxy in each bin is determined with respect to the galaxy distance from the filament skeleton

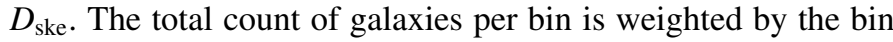
volume, in a similar way as making a normalized histogram. In order to compare samples of different sizes, a normalization is applied by dividing the number of events in a bin by the total number of galaxies in the sample.

\section{Galaxy System-Finding algorithm (GSyF)}

\subsection{Detection of high-density regions}

We first searched for the high-relative-density regions, clusters, and groups of galaxies (which we refer to generically as galaxy systems) inside the studied superclusters, because these systems are the natural nodes for filaments. This was also necessary for correcting the FoG effect and having the data prepared for the application of the filament-finding algorithm (see following section). Furthermore, the detection of galaxy systems allows the identification of new and possibly previously unknown systems (especially poorer galaxy groups), and the improvement of the membership estimation of the superclusters themselves.

A description of the algorithm, including the strategy we used for optimizing its parameters using simulated mock volumes, is presented in Santiago-Bautista et al. (2019). Here we review the main steps of this algorithm. First we calculate the local surface density at the position of each galaxy in the projected area of the supercluster by applying the VT method (Sect. 3.1). The VT individual area of the galaxy can be directly converted to a surface density estimation $\left(d_{i}=1 / a_{i}\right)$, in this case in units of $\mathrm{deg}^{-2}$. It is worth noting that the boxes we considered for the superclusters in our sample comprehend slices in redshift-space in the range $0.02 \leq \Delta z \leq 0.07$.

In order to identify the galaxy systems, we start by applying the HC method (Sect. 3.2), but only to the $N_{\text {gal }}$ galaxies with densities above a baseline density, $d_{\text {bas }}$, which should be analogous to a background density. In a certain sense, this separates supercluster galaxies from void galaxies (i.e., under-dense regions). This baseline is calculated from the mean density by randomizing the galaxies in each sky-projected area. Since the distribution of points in space is not isotropic, it is not possible to directly set a background density from the projected positions of the galaxies. Therefore, it is necessary to simulate an isotropic distribution of the points in order to set the baseline value (see, e.g., Cybulski et al. 2014). A set of 1000 randomizations of the point positions is generated, each with the same sample number over the same area. The mean surface density is then calculated using:

$d_{\mathrm{bas}}=\frac{1}{m} \sum_{j=1}^{m} \frac{1}{n} \sum_{i=1}^{n} d_{i, j}^{\prime}$,

where $d_{i, j}^{\prime}=1 / a_{i, j}^{\prime}$ corresponds to the inverse of the area of the point $x_{i}^{\prime}$ for the randomization $j$.

Since the distribution of galaxies is not homogeneous among the different boxes, we calculate independent baseline values for each supercluster; see Table 2 . A density contrast $\left(\delta_{i}\right)$ is then calculated as:

$\delta_{i}=\frac{d_{i}-d_{\mathrm{bas}}}{d_{\mathrm{bas}}}$.

Here, the $N_{\text {gal }}$ galaxies to which we apply the $\mathrm{HC}$ are the ones with a density contrast, $\delta_{i}>0$ (see Santiago-Bautista et al. 2019, for an evaluation of the negligible effect of slightly changing the density threshold).

Subsequently, we apply HC to the set of parameters (RA, Dec, $1000 z$ ) for these galaxies (the factor of 1000 is the weight for $z$ values to be comparable to the sky coordinates values). The number of groups taken from the analysis is defined as a cut of the HC tree, fixed to $N_{\text {cut }}=N_{\text {gal }} / f$, with a segmentation parameter $f$, which is the expected mean number of elements per group. Currently, the selection of the optimal number of groups in clusterization methods is still a topic under investigation in the pattern-recognition community, which includes the $\mathrm{HC}$ algorithm. A specific value of $f$ was calculated for each supercluster ( $3 \leq f \leq 36$ ) according to the optimization process described in Santiago-Bautista et al. (2019). This strategy was adopted because a physically motivated value for $f$ would depend on many parameters, like the density of galaxies in each box, the sampling of these galaxies with respect to the real distribution, and the redshift, among others, which are difficult to estimate for our data.

Finally, we select only those systems with a number of galaxies, $N_{j}$, larger than two. These pre-identified systems are then subjected to the next step of refinement: the iterative estimation of the dynamical parameters, virial mass and radius.

\subsection{Finger of God correction}

After identifying the galaxy systems, we proceed to refining the galaxy membership and correcting the galaxy positions for the FoG effect using a virial approximation. We apply a simplified version of the algorithm presented by Biviano et al. (2006) for the estimation of the virial mass and radius. We do not apply the surface pressure term correction based on the concentration parameter. Avoiding such a correction can lead to an overestimation of the virial radius, but for this geometric analysis, a virial approximation is enough.

The virial-parameter-calculation algorithm works as follows: First we take the projected center and mean velocity of the system from the results of $\mathrm{HC}\left(C_{j}\right)$. The projected center is then set at the position of the brightest $r$-band magnitude member galaxy (BMG) within $1 \sigma_{j}$ from the $\mathrm{HC}$ center, while the $\mathrm{HC}$ mean velocity is used directly. Those galaxies that are expected to belong to the system are selected among all galaxies in the sample (those with spectroscopic redshift in SDSS-DR13) that are projected inside a cylinder of radius $R_{a}$ (hereafter referred to as the aperture). Biviano et al. (2006) show that the dynamical analyses are similar for different aperture sizes. We chose an aperture of $R_{a}=1 \mathrm{~h}_{70}^{-1} \mathrm{Mpc}$. Subsequently, for the line-of-sight 


\section{GSyF Algorithm}

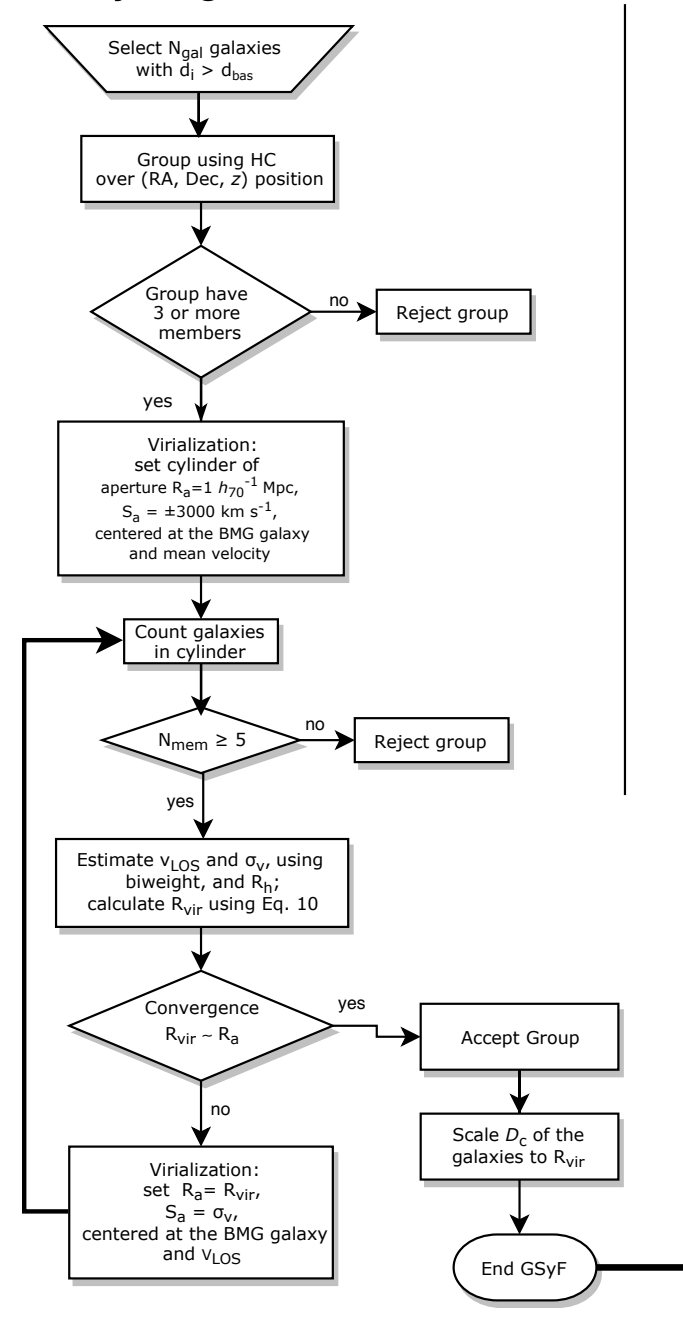

\section{GFiF Algorithm}

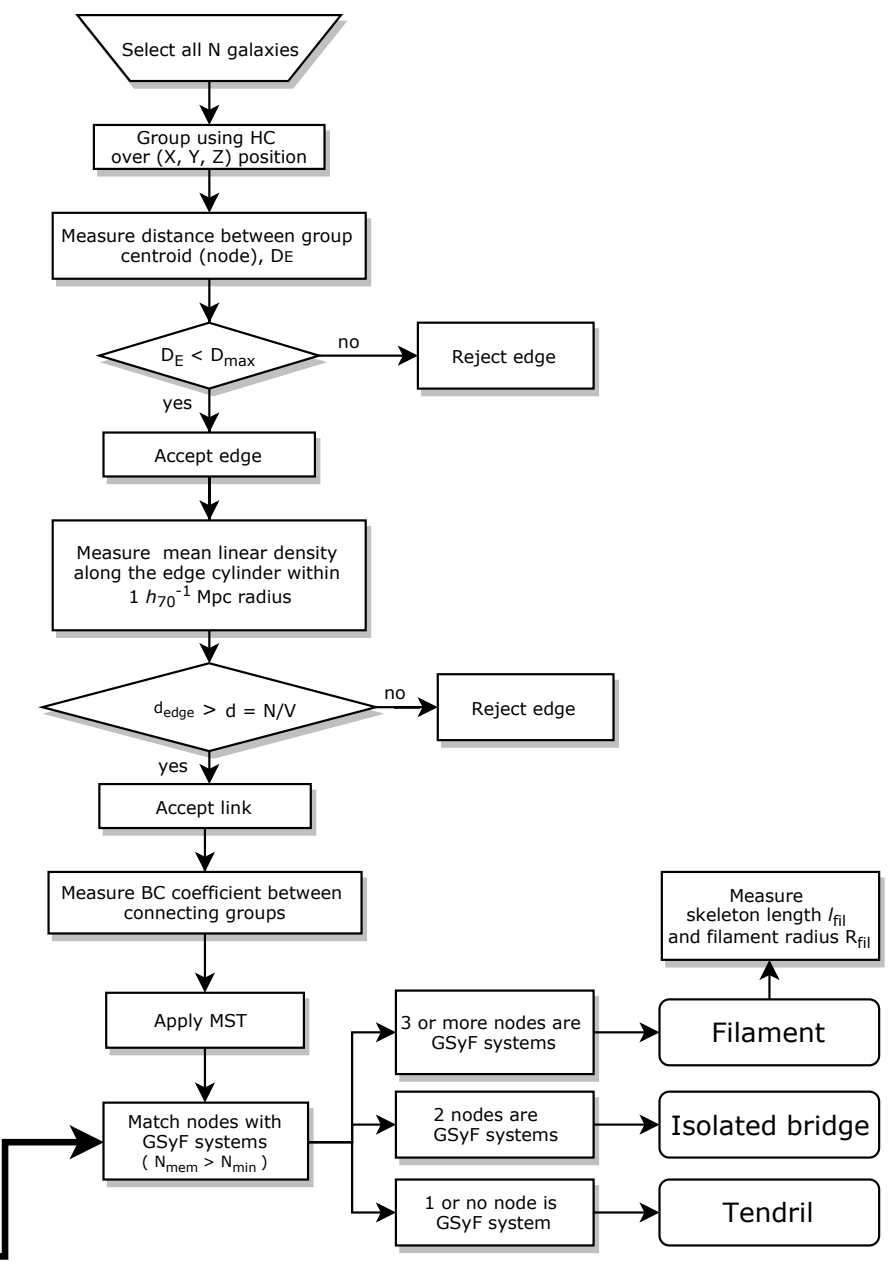

Fig. 2. Flow chart of the GSyF (left side) and GFiF (right side) algorithms.

direction, we select galaxies with a difference in velocity of up to $S_{a}= \pm 3000 \mathrm{~km} \mathrm{~s}^{-1}$ with respect to the mean cluster velocity. This would correspond to three times the velocity dispersion of a rich cluster. A robust estimation of mean velocity, $v_{\mathrm{LOS}}$, and velocity dispersion, $\sigma_{v}$, for the galaxies inside the cylinder is obtained using Tukey's biweight method (Beers et al. 1990). An approximation of the mass $M_{a}$ in the aperture is computed as:

$M_{a}=\frac{3 \pi}{2 G} \sigma_{v}^{2} R_{h}$,

where $G$ is the gravitational constant, $3 \pi / 2$ is the deprojection factor, and $R_{h}$ is the projected harmonic radius.

We calculate the virial radius, $R_{\mathrm{vir}}^{3}=(3 / 4 \pi)\left(M_{\mathrm{vir}} / \rho_{\mathrm{vir}}\right)$, by assuming a spherical model for nonlinear collapse, that is, by taking the virialization density as $\rho_{\text {vir }}=18 \pi^{2}\left[3 H^{2}(z)\right] /[8 \pi G]$, and $M_{a}$ as an estimation for $M_{\mathrm{vir}}$. We thus obtain

$R_{\mathrm{vir}}^{3}=\frac{\sigma_{v}^{2} R_{h}}{6 \pi H^{2}(z)}$.

The aperture $R_{a}$ is then updated to the calculated $R_{\text {vir }}$ value, the mean velocity to $v_{\mathrm{LOS}}$, and $S_{a}$ to $\sigma_{v}$, defining a new cylinder. This process is repeated iteratively until the radius $R_{\mathrm{vir}}$ converges. $M_{\text {vir }}$ is finally calculated at the end of the iteration process.

The correction for the FoG effect is carried out by adjusting the position of the $N_{\text {mem }}$ galaxies inside the final cylinder. This is done by scaling their comoving distances along the cylinder to the calculated virial radius. A schematic representation of the GSyF algorithm, including the FoG correction, can be found on the left side of Fig. 2.

\section{Galaxy Filaments skeleton-Finding algorithm (GFiF)}

\subsection{Detection of low-density regions}

As we are interested in the detection and analysis of elongated and low-relative-density contrast structures, we apply again a combined VT+HC method to the data, but now in the rectangular 3D space, with the positions of the galaxies corrected for the FoG effect. Thus, the VT densities are now volume densities, in units of $\mathrm{Mpc}^{-3}$. At the beginning of this analysis the $\mathrm{HC}$ method is applied to all galaxies in the volume without density restrictions, that is, no baseline is applied. Density restrictions are considered later as criteria for the construction of filaments. Another difference between this application of $\mathrm{VT}+\mathrm{HC}$ and the one used for the GSyF methodology is a relaxed cut in the hierarchical tree. Since we are interested in detecting more elongated representative structures, we tested values for the segmentation parameter $f$ between 10 and 40 . The direct effect of relaxing the cut is to allow the detection of groups at lower densities. 


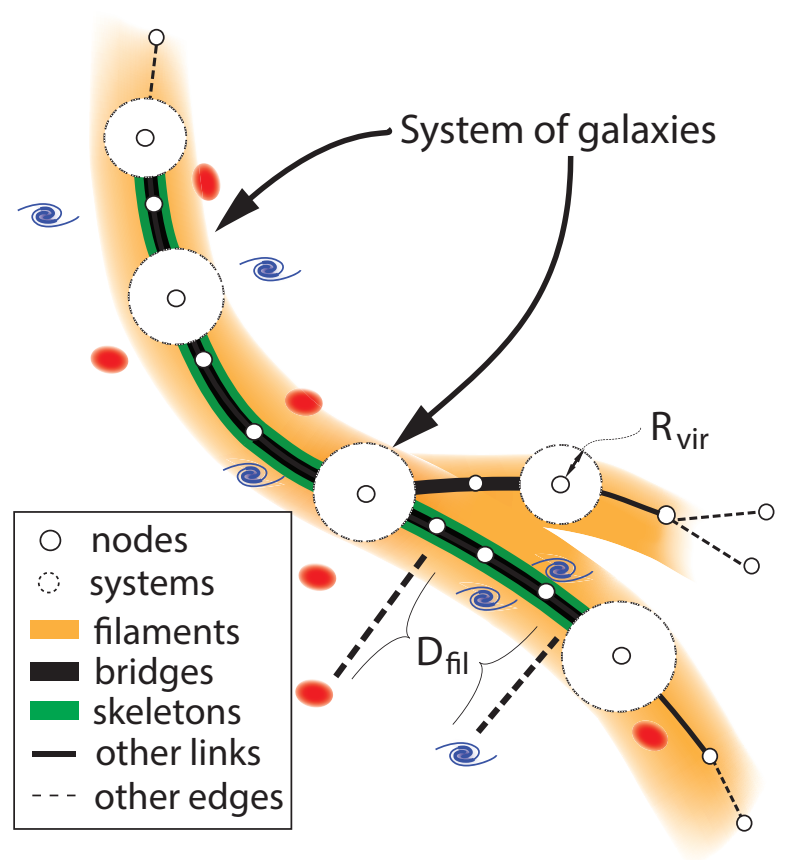

Fig. 3. Representation of a filament. Graph nodes are represented by white circles and edges by dark lines. The five systems connected are represented by a dotted circle of radius $R_{\mathrm{vir}}$. A bridge connecting two systems is represented as a bold black line. The distance from galaxies to the filament (bold dashed line) is measured along a line perpendicular to the edges.

Here we need to make some practical definitions in order to describe our strategy. Figure 3 shows the following definitions schematically.

- The nodes to which we apply the method correspond, in the context in which we are working, to the $\mathrm{HC}$ group centroids.

- An edge is defined as any connection between two nodes.

- The real "links" between the systems are defined as the most promising edges, filtered according to their proximity and density contrast.

- Spanning trees are extracted as described in Sect. 3.4, by cutting the graph in uncycled optimal trees. Some nodes inside a spanning tree may have been detected as galaxy systems by the GSyF algorithm.

- A "bridge" is defined as a sequence of links and nodes between two systems.

- A "filament" is identified if a spanning tree bridges three or more systems connected by bridges.

- If the spanning tree contains zero or only one system, it is called a "tendril".

- The "skeleton" is the medial line of a filament. The method for finding it, which intends to reduce the dimensionality of the objects (in our case, galaxy filaments), is known as "skeletonization”.

\subsection{Chaining the filaments}

Once we have applied HC, we measure the Euclidean distance $D_{\mathrm{E}}$ of the centroid (node) of each group against all its group neighbors. These connections (edges) can be represented by an undirected graph as described in Sect. 3.3. The weights $W$ of the edges are set by the Bhattacharyya coefficient, BC, defined as:
$\mathrm{BC}\left(P_{1}, P_{2}\right)=\sum_{x \in X} \sqrt{P_{1}(x) P_{2}(x)}$.

The Bhattacharyya coefficient quantifies the amount of overlapping between two distributions $P_{1}(x)$ and $P_{2}(x)$. Thus, the orientation of the two groups weights the connection between them.

In the next step, we filter the edges by two criteria: First we select the edges corresponding to a $D_{\mathrm{E}}$ smaller than a threshold, $D_{\max }$ (hereafter, linking length). Secondly, we consider an edge as a real link of galaxies based on the following: (i) we define a cylinder along the edge with a radius of $1 \mathrm{~h}_{70}^{-1} \mathrm{Mpc}$; (ii) we measure the linear density of galaxies along the cylinder; and (iii) if the mean linear density of the cylinder is above $d=N / V$ (Table 2) we take the cylinder as a link of galaxies connecting the two nodes.

Each ensemble of connected links is a tree in the forest graph. We then apply Kruskal's MST technique (Sect. 3.4) to the forest graph to identify independent trees and their dominant branches. To proceed we need to match the list of detected spanning trees with the list of detected GSyF systems. However, due to the effect of losing sampled galaxies with increasing redshift (see Fig. 1), the richness of the detected systems depends on the redshift. In other words, to have a comparable richness for two similar systems, for instance one at $z=0.03$ and another at $z=0.13$, we have to apply a correcting factor to the richness of the second one. To overcome this limitation, we apply the following lower limit for the richness of the systems at the supercluster redshift: $\log _{10} N_{\min }=a \log _{10} z+b$, with $a=-1.0$ and $b=-0.2$. This leads to a lower richness limit of $N_{\min }=30$ 5 galaxies per system, from the nearest and farthest supercluster in our sample respectively.

Now that we have the systems, and the bridges between them (instead of the nodes and edges in the previous step), we can identify the filaments. As stated above, and following the definition by Chow-Martínez et al. (in prep.), we search for the filaments which have at least three galaxy systems connected by bridges.

Although isolated bridges (i.e., connecting only one pair of systems) and tendrils (connections between nodes with no system embedded) are important and are also a byproduct of the algorithm, we focus our discussion hereafter on the filaments. The connecting edges of these filaments are then refined using Dijkstra's algorithm (Sect. 3.5). This refinement allows the identification of the filament skeleton, that is, the principal branch connection. According to the pattern-recognition literature, a skeleton represents the principal features of an object such as topology, geometry, orientation, and scale. Figure 4 presents the steps of the GFiF algorithm schematically.

The results of the filament-finding algorithm depend on several parameters, in particular the number of $\mathrm{HC}$ groups, $N_{\text {cut }}$ (or, equivalently, $f$ ), and the linking length $D_{\max }$. Therefore, it is necessary to carry out a search for the optimal combination of these parameters. In addition, in order to find the longest filaments possible inside the supercluster volume, we search for the linking length that maximizes the number of filaments in the box, that is just before they begin to percolate. The optimization for these parameters is described in detail in Santiago-Bautista et al. (2019). We found that the optimized parameter $f$ decreases with $z$ from about 20 to 10 galaxies in the range covered by our sample, that is, depending on the sampling, a smaller density is found with increasing $z$. The $D_{\max }$, in turn, increases with $z$, in such a way as to compensate for the decrease in $f$. A schematic representation of the GFiF algorithm can be found on the right side of Fig. 2. 

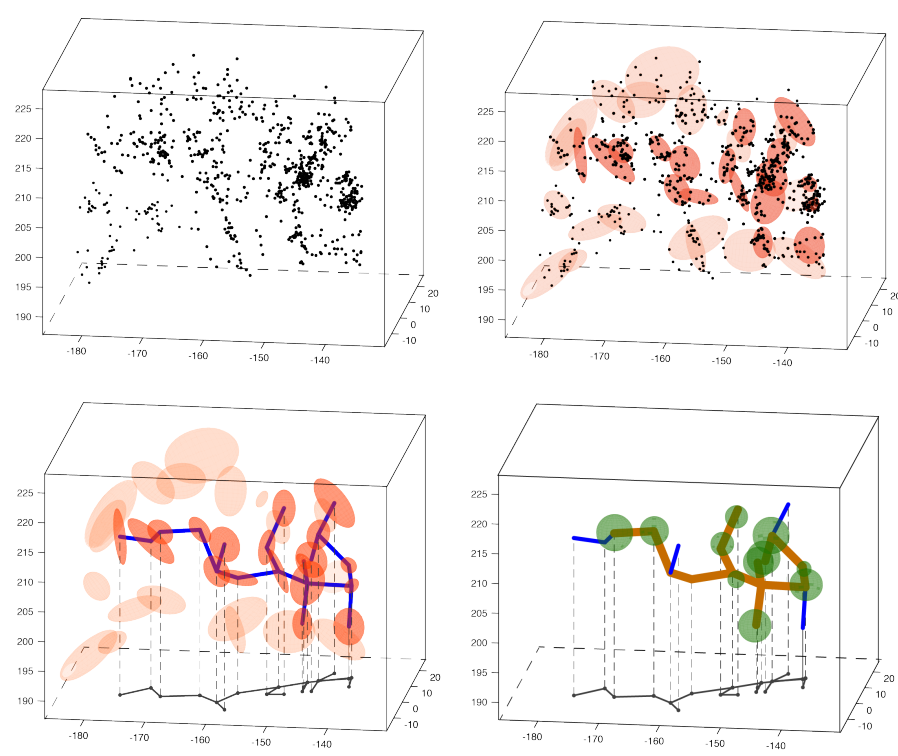

Fig. 4. Illustration of the steps of the GFiF algorithm. In the first box (top-left) one can see the distribution of galaxies. In the second one (top-right) the $\mathrm{HC}$ groups are marked, with denser red colors representing the richer HC groups. The filtered edges (links) among the groups of the spanning tree are displayed in the third box (bottom-left). The last box (bottom-right) presents the systems (green circles), bridges (brown lines), and other links (blue lines) found among the groups of the preceding step.

\section{Detection algorithms in action}

In order to illustrate the function of the detection algorithms presented above, we now describe their application to one of the superclusters in our sample, MSCC 310, the Ursa-Majoris Supercluster (see Tables 1 and 2). This supercluster contains 21 Abell clusters with redshifts in the range from 0.05 to 0.08 and is one of the largest in volume in our sample. MSCC 310 occupies an area in the sky of about $1700 \mathrm{deg}^{2}$, equivalent to a volume of $\left(116 h_{70}^{-1} \mathrm{Mpc}\right)^{3}$ - including the $20 \mathrm{~h}_{70}^{-1} \mathrm{Mpc}$ added to the box limits from the farthest clusters.

The volume contains $N=12286$ SDSS galaxies with spectroscopic redshift. This corresponds to a mean surface density of 76.9 gal. $\mathrm{deg}^{-2}$ or 0.008 gal.h $_{70}^{3} \mathrm{Mpc}^{-3}$; see Table 2. The list of parameter values used is shown in Table 3.

\subsection{Application of GSyF to MSCC 310}

First we applied the VT algorithm to the projected distribution of MSCC 310 galaxies and calculated their $d_{i}$. We then made 1000 simulations to estimate $d_{\text {bas }}\left(15.7 \mathrm{deg}^{-2}\right.$, for this case). We applied the $\mathrm{HC}$ algorithm to the $N_{\mathrm{gal}}=7529$ galaxies with $\delta_{i}>0$. After calculating the best $f$ parameter from the 30 mock simulations ( $f=6$, in this case), we took the $N_{\text {cut }}=1140$ groups generated from the $\mathrm{HC}$ application. As expected, these groups have, approximately six members on average. Of these, we retained 1015 with $N_{j} \geq 3$.

The iterative virial refinement was initialized by assigning the center of each HC group to the brightest $r$-band galaxy member close to its geometrical centroid (see Table 4). For MSCC 310 groups, the mean difference between the geometrical center and the projected position of the brightest galaxy was found to be about $350 \mathrm{~h}_{70}^{-1} \mathrm{kpc}$. On average, virial refinement required six iterations in order to produce convergence to the virial radius. This refinement resulted in 122 systems with
$N_{\text {mem }} \geq 10$ for the MSCC 310 volume. The refinement also detected 113 smaller systems with $5 \leq N_{\text {mem }}<10$.

In Table 4 we list the properties of the first 25 richest systems for the MSCC 310 supercluster. The range of virial radii of the GSyF systems with $N_{\text {mem }} \geq 10$ in MSCC 310 was $0.7-2.5 \mathrm{~h}_{70}^{-1} \mathrm{Mpc}$. For groups with $5 \leq N_{\text {mem }}<10$, the range of virial radius lies within $0.4-0.9 \mathrm{~h}_{70}^{-1} \mathrm{Mpc}$. After the refinement, the projected central position of the systems changed by $170 \mathrm{~h}_{70}^{-1} \mathrm{kpc}$ on average, while the redshift was refined for some cases up to $\Delta z \sim 0.001$ or $\Delta \sigma_{v} \sim 300 \mathrm{~km} \mathrm{~s}^{-1}$.

As an example, the richest system in MSCC 310 is the cluster A1291 A. Its HC initial centroid position (set as the position of the brightest galaxy in the HC group: $\alpha=172.73, \delta=56.49$ and $z=0.0611$ ) changed by $13 \mathrm{~h}_{70}^{-1} \mathrm{Mpc}$ after 17 iterations of the virial refinement (the final centroid position corresponds to $\alpha=$ $173.01, \delta=56.09, z=0.0535)$. This position is at $240 \mathrm{~h}_{70}^{-1} \mathrm{kpc}$ from the system's brightest galaxy detected for A1291 A which has coordinates $(\alpha=173.05, \delta=56.05, z=0.0585$; Lauer et al. 2014).

Finally, as described in Sect. 4.2, we correct the $D_{\mathrm{C}}$ of the member galaxies in each system by re-scaling their dispersion range to the $R_{\mathrm{vir}}$ of the system. An example of the MSCC 310 volume before and after the correction is shown in Fig. 5.

\subsection{Application of GFiF to MSCC 310}

With the co-moving distances for the MSCC 310 galaxies corrected for the FoG effect, we proceeded to transform their sky coordinates to rectangular ones following Eqs. (1)-(3). The $N_{\text {gal }}$ was now taken to be the total number of galaxies in the box of MSCC 310, $N=12286$, for which we applied the GFiF method. The VT algorithm was then applied to calculate volumetric numerical densities. We used a segmentation parameter of $f=16$ for the HC algorithm. From that, we identified 768 low-density groups and for each pair we calculated the $D_{\mathrm{E}}$ distance between centers and the $\mathrm{BC}$ weight. As expected, the implementation of the $\mathrm{HC}$ algorithm over all galaxies detected larger groups $(\sim 15$ galaxies on average now) and those were more elongated, with a mean $\sigma_{j}$ of $1.8 \mathrm{~h}_{70}^{-1} \mathrm{Mpc}$ compared with the mean $\sigma_{j}$ of $0.5 \mathrm{~h}_{70}^{-1} \mathrm{Mpc}$ found with the application of GSyF.

In order to filter the connections, a linking length of $D_{\max }=$ $8 \mathrm{~h}_{70}^{-1} \mathrm{Mpc}$ was used resulting in 334 edges. As described above, $D_{\max }$ and $f$ were obtained by the optimization process described in Santiago-Bautista et al. (2019). The second filter, the minimum mean linear density along the edge cylinders (in this case 0.008 gal. $\mathrm{Mpc}^{-3}$ ), left 273 links from the 316 connections smaller than $D_{\max }$. This resulted in 34 trees, to which we applied MST. Of these, only 9 are linking three or more systems of galaxies with a richness $N_{\text {mem }}$ above 11 galaxies, and the rest are isolated bridges and tendrils. This result is shown in the dendrogram depicted in Fig. 6 (top panel) which shows nine dominant filaments for the MSCC 310 supercluster.

Concerning the systems embedded in the structures, from the $359 \mathrm{HC}$ groups (nodes) in the spanning trees, 116 were found to have a match in the systems with $N_{\text {mem }} \geq 10$ identified with GSyF. From these, 61 were found to be in filaments (53\%), 26 (22\%) in bridges between pairs of systems, and 29 (25\%) were found to not be connected by bridges, that is, they were relatively isolated. The filaments detected by the GFiF algorithm in the MSCC 310 supercluster and their main properties are listed in Table 5.

We can also observe the filaments inside the MSCC 310 volume in the bottom panel of Fig. 6. In this panel, the nine filaments are plotted over the distribution of galaxies in a $\mathrm{RA}[\mathrm{deg}] \times \mathrm{Z}[\mathrm{Mpc}]$ plane. This projection allows the recognition 
Table 3. Glossary of parameters used by GSyF and GFiF algorithms.

\begin{tabular}{|c|c|c|}
\hline Param. & Description & MSCC 310 \\
\hline \multicolumn{3}{|c|}{ Properties of the rectangular box } \\
\hline$N$ & Total number of galaxies & 12286 \\
\hline$V$ & Total volume & $\left(116 \mathrm{~h}_{70}^{-1} \mathrm{Mpc}\right)^{3}$ \\
\hline$d=\frac{N}{V}$ & Mean volumetric number density & $0.008 \mathrm{~h}_{70}^{3} \mathrm{Mpc}^{-3}$ \\
\hline$A$ & Projected area in the sky & $(12.65 \mathrm{deg})^{2}$ \\
\hline$d_{\text {surf }}=\frac{N}{A}$ & Mean surface number density (sky projection) & $76.9 \mathrm{deg}^{-2}$ \\
\hline$v_{i}, a_{i}$ & Local volume or projected area of VT cell & Each galaxy \\
\hline$d_{i}$ & Local VT (surface or volume) density & Each galaxy \\
\hline$D_{\mathrm{C}}$ & Comoving distance of the galaxy or system & Each galaxy or system \\
\hline$D_{\mathrm{E}}$ & Euclidean distance between two galaxies or two nodes (edge size) & Each pair \\
\hline$D_{\text {ske }}$ & Euclidean distance of galaxy from filament skeleton & Each galaxy \\
\hline \multicolumn{3}{|c|}{ Amount of systems } \\
\hline$N_{\mathrm{Cl}}$ & Richness of the supercluster (nr. of Abell/ACO clusters) & 21 \\
\hline$N_{\text {cut }}=\frac{N_{\text {gal }}}{f}$ & No. of extracted HC groups & 1140,768 \\
\hline$N_{\mathrm{HC}}$ & No. of detected HC groups $\left(N_{j} \geq 3\right)$ found by GSyF algorithm & 1015 \\
\hline$N_{\text {nodes }}$ & No. of nodes found by GFiF algorithm which remained in the MSTrees & 359 \\
\hline$N_{\text {FoG }}$ & No. of systems (that survived the FoG filter) & 255 \\
\hline$N_{\text {sys }}$ & No. of systems with $N_{\text {mem }}>N_{\text {min }}$ in box which matched nodes & 116 \\
\hline$N_{\text {sfil }}$ & No. of systems embedded in the filaments & 61 \\
\hline$N_{\text {spair }}$ & No. of systems forming pairs connected by isolated bridges & 26 \\
\hline$N_{\text {sout }}$ & No. of systems not forming filaments or pairs & 29 \\
\hline \multicolumn{3}{|c|}{ Amount of filaments, isolated bridges and tendrils } \\
\hline$N_{\text {fil }}$ & No. of filament candidates of the supercluster & 4 \\
\hline$N_{\text {ske }}$ & No. of detected filaments in the box (linked bridges) & 9 \\
\hline$N_{\text {brid }}$ & No. of detected isolated bridges between two systems only & 17 \\
\hline$N_{\text {tend }}$ & No. of detected tendrils (not bridges or filaments) & 18 \\
\hline \multicolumn{3}{|c|}{ Properties of the systems } \\
\hline$P_{j}(x)$ & Gaussian model for detected $\mathrm{HC}$ group & Each HC group \\
\hline$N_{j}$ & Richness the detected HC group & Each HC group \\
\hline$C_{j}$ & Centroid position of the detected $\mathrm{HC}$ group & Each HC group \\
\hline$\sigma_{j}$ & Compactness (covariance) of the detected HC group & Each HC group \\
\hline$C_{B M G}$ & Position of Brightest HC group Member Galaxy & Each HC group \\
\hline$C_{\mathrm{FoG}}$ & Centroid position of the detected FoG system & Each system \\
\hline$N_{\text {mem }}$ & Richness (nr. of galaxies) of the detected FoG system & Each system \\
\hline$N_{\min }$ & Minimum nr. of galaxies for systems in filaments at different $z$ & 10 \\
\hline$R_{h}$ & Harmonic radius of the detected FoG system & Each system \\
\hline$R_{\mathrm{vir}}$ & Virial radius of the detected FoG system & Each system \\
\hline$M_{\text {vir }}$ & Virial mass of the detected FoG system & Each system \\
\hline$v_{\mathrm{LOS}}$ & Robust line-of-sight velocity of the detected FoG system & Each system \\
\hline$\sigma_{v}$ & Robust velocity dispersion of the detected FoG system & Each system \\
\hline \multicolumn{3}{|c|}{ Properties of the filaments } \\
\hline$N_{\text {edges }}$ & No. of edges that survived filter 1 & 334 \\
\hline$N_{\text {links }}$ & No. of edges that survived filters 1 and 2 & 316 \\
\hline$N_{\text {trees }}$ & No. of trees after MST & 17 \\
\hline$N_{\text {nod }}$ & No. of nodes in the filament (or in the skeleton) & Each filament (skeleton) \\
\hline$\ell_{\text {fil }}$ & Length of filament skeleton & Each filament \\
\hline$R_{\mathrm{fil}}$ & Mean radius of the filament & Each filament \\
\hline$d_{\text {fil }}$ & Mean galaxy number density inside the filament & Each filament \\
\hline$N_{\text {gfil }}$ & No. of galaxies hosted in the filaments of the box & 2568 \\
\hline$V_{\text {fil }}$ & Volume occupied by the filaments of the box & $\left(1.16 \mathrm{~h}_{70}^{-1} \mathrm{Mpc}\right)^{3}$ \\
\hline \multicolumn{3}{|c|}{ GSyF and GFiF parameters } \\
\hline$d_{\text {bas }}$ & Projected number density baseline & $15.7 \mathrm{deg}^{-2}$ \\
\hline$\delta_{i}$ & Local density contrast & Each galaxy \\
\hline$N_{\text {gal }}$ & No. of galaxies above the baseline $d_{\text {bas }}$ & 6842 \\
\hline$R_{a}, S_{a}, M_{a}$ & Parameters of iterative process for FoG correction & Each HC group \\
\hline$f$ & Segmentation parameter (OPTIMIZATION) & 6,16 \\
\hline $\mathrm{BC}$ & Bhattacharyya coefficient (edge weight) & Each edge \\
\hline$D_{\max }$ & Linking length (first filter) (OPTIMIZATION) & $8 \mathrm{~h}_{70}^{-1} \mathrm{Mpc}$ \\
\hline$d_{\text {edge }}$ & Edge cylinder density (second filter) & Each edge \\
\hline$R_{\mathrm{cy}}$ & Filament concentric cylinder radius & Each filament \\
\hline
\end{tabular}


Table 4. Main properties of the 25 richest systems identified in the volume of the supercluster MSCC 310.

\begin{tabular}{|c|c|c|c|c|c|c|c|c|c|c|c|}
\hline \multirow{2}{*}{$\begin{array}{l}\text { System } \\
\text { Nr. } \\
(1)\end{array}$} & \multirow{2}{*}{$\begin{array}{c}N_{\text {mem }} \\
(2)\end{array}$} & \multicolumn{3}{|c|}{$C_{\mathrm{BMG}}$} & \multicolumn{3}{|c|}{$C_{\mathrm{FoG}}$} & \multirow{2}{*}{$\begin{array}{r}\sigma_{v} \\
{\left[\mathrm{~km} \mathrm{~s}^{-1}\right]} \\
(9)\end{array}$} & \multirow{2}{*}{$\begin{array}{c}R_{h} \\
{\left[\mathrm{~h}_{70}^{-1} \mathrm{Mpc}\right]} \\
(10)\end{array}$} & \multirow{2}{*}{$\begin{array}{c}R_{\mathrm{vir}} \\
{\left[\mathrm{h}_{70}^{-1} \mathrm{Mpc}\right]} \\
\quad(11)\end{array}$} & \multirow{2}{*}{$\begin{array}{l}\text { cross-ref } \\
\text { ACO Nr } \\
(12)\end{array}$} \\
\hline & & $\begin{array}{c}\mathrm{RA}_{J 2000} \\
\text { (3) }\end{array}$ & $\begin{array}{c}\operatorname{Dec}_{J 2000} \\
(4)\end{array}$ & $\begin{array}{c}z \\
(5)\end{array}$ & $\begin{array}{c}\mathrm{RA}_{J 2000} \\
(6)\end{array}$ & $\begin{array}{c}\operatorname{Dec}_{J 2000} \\
(7)\end{array}$ & $\begin{array}{c}z_{\mathrm{LOS}} \\
(8)\end{array}$ & & & & \\
\hline 1 & 123 & 173.09667 & 55.96744 & 0.0515 & 173.01489 & 56.09476 & 0.0535 & 1182 & 1.09 & 2.54 & A1291 A \\
\hline 2 & 103 & 174.01464 & 55.07526 & 0.0571 & 174.17793 & 55.19984 & 0.0587 & 1103 & 1.28 & 2.56 & A1318 A \\
\hline 3 & 95 & 180.26970 & 56.37019 & 0.0648 & 180.07048 & 56.20431 & 0.0649 & 762 & 1.04 & 1.87 & A1436 \\
\hline 4 & 94 & 167.09625 & 44.15030 & 0.0587 & 167.10957 & 44.07194 & 0.0590 & 644 & 0.81 & 1.53 & A1169 \\
\hline 5 & 91 & 176.83909 & 55.73018 & 0.0515 & 176.80652 & 55.69322 & 0.0518 & 712 & 0.80 & 1.64 & A1377 \\
\hline 6 & 82 & 177.19063 & 54.51936 & 0.0601 & 177.05852 & 54.64399 & 0.0604 & 845 & 0.95 & 1.94 & A1383 \\
\hline 7 & 67 & 168.84947 & 54.44412 & 0.0695 & 168.90590 & 54.50901 & 0.0700 & 659 & 0.91 & 1.63 & \\
\hline 8 & 61 & 175.27722 & 55.18836 & 0.0593 & 175.25812 & 55.29493 & 0.0609 & 1103 & 1.30 & 2.58 & A1349 A \\
\hline 9 & 61 & 163.40237 & 54.86794 & 0.0716 & 163.54104 & 54.84312 & 0.0722 & 640 & 0.89 & 1.58 & \\
\hline 10 & 59 & 172.33060 & 54.12608 & 0.0689 & 172.44575 & 54.08347 & 0.0690 & 582 & 0.75 & 1.40 & A1270 \\
\hline 11 & 54 & 158.24537 & 56.74813 & 0.0448 & 158.32527 & 56.82434 & 0.0454 & 459 & 0.69 & 1.16 & \\
\hline 12 & 52 & 180.22849 & 51.42263 & 0.0666 & 180.46535 & 51.65240 & 0.0649 & 1069 & 1.08 & 2.37 & A1452 \\
\hline 13 & 50 & 152.32009 & 54.21099 & 0.0465 & 152.41409 & 54.42037 & 0.0460 & 415 & 0.68 & 1.08 & \\
\hline 14 & 46 & 183.70265 & 59.90619 & 0.0600 & 183.59629 & 59.90299 & 0.0599 & 443 & 0.75 & 1.17 & A 1507 B \\
\hline 15 & 43 & 168.06955 & 57.07599 & 0.0471 & 168.12753 & 57.04832 & 0.0467 & 491 & 0.77 & 1.26 & \\
\hline 16 & 42 & 178.37743 & 52.68944 & 0.0716 & 178.59933 & 52.77017 & 0.0695 & 761 & 0.84 & 1.74 & \\
\hline 17 & 39 & 151.21598 & 54.56786 & 0.0470 & 150.99614 & 54.65456 & 0.0472 & 460 & 0.64 & 1.13 & \\
\hline 18 & 39 & 163.28303 & 56.33167 & 0.0772 & 163.35966 & 56.33847 & 0.0745 & 1003 & 0.89 & 2.13 & \\
\hline 19 & 36 & 172.42861 & 55.38047 & 0.0685 & 172.44796 & 55.42240 & 0.0684 & 534 & 0.54 & 1.19 & \\
\hline 20 & 36 & 162.94746 & 55.38567 & 0.0739 & 162.89992 & 55.34739 & 0.0737 & 367 & 0.68 & 1.00 & $\mathrm{~A} 1112 \mathrm{~A}$ \\
\hline 21 & 34 & 182.19381 & 53.33371 & 0.0813 & 182.19494 & 53.31805 & 0.0821 & 573 & 0.66 & 1.33 & \\
\hline 22 & 33 & 181.31486 & 43.16902 & 0.0528 & 181.40583 & 43.20470 & 0.0526 & 504 & 0.67 & 1.23 & \\
\hline 23 & 31 & 177.05076 & 52.85209 & 0.0503 & 177.04947 & 52.59945 & 0.0505 & 556 & 0.67 & 1.31 & \\
\hline 24 & 31 & 178.57105 & 55.47082 & 0.0508 & 178.68334 & 55.20458 & 0.0513 & 584 & 0.82 & 1.45 & \\
\hline 25 & 31 & 151.31217 & 53.14899 & 0.0463 & 151.31677 & 52.99140 & 0.0451 & 431 & 0.68 & 1.11 & \\
\hline
\end{tabular}

Notes. The columns in the table are as follows: (1) sequential number assigned to the system according to its richness; (2) richness of the system; (3) RA coordinate of the brightest member of the system; (4) Dec coordinate of the brightest member of the system; (5) redshift of the brightest member of the system; (6) RA coordinate of the final system centroid; (7) Dec coordinate of the final system centroid; (8) redshift of the final system centroid; (9) velocity dispersion calculated for the system; (10) harmonic radius calculated for the system; (11) virial radius calculated for the system; (12) cross-reference with Abell clusters. The complete version of this table and the tables of systems of the other superclusters are available at the CDS.

of structures both in one of the coordinates of the sky plane and depth. Filaments are depicted in the same colors in both panels of this figure. Isolated bridges, which connect two systems alone without forming a filament, are represented only in the bottom panel and by black lines. Tendrils are not represented to avoid crowding. The longest paths for the filament skeletons, that is, those that connect the farthest systems of each filament, range from 18 to $62 \mathrm{~h}_{70}^{-1} \mathrm{Mpc}$ and connect up to 11 systems inside the MSCC 310 volume. Moreover, we measured the paths between pairs of systems chained together by bridges; such distances range from 5 to $24 \mathrm{~h}_{70}^{-1} \mathrm{Mpc}$.

\section{Validation of the methods}

\subsection{Checking the identified systems of galaxies}

In order to validate our GSyF algorithm we compared the list of identified systems to different cluster and group catalogs in the region of SDSS. For MSCC 310, for instance, GSyF detected 122 systems with ten or more galaxies and another 113 systems with $5 \leq N_{\text {mem }}<10$. A match was considered positive if the projected positions of the system in the two compared catalogs were found to be within $1 \mathrm{~h}_{70}^{-1} \mathrm{Mpc}$ of one another, while in redshift space we considered a difference $\Delta z=0.007$ which corresponds to $\pm 2100 \mathrm{~km} \mathrm{~s}^{-1}$.
For the rich clusters, first we compared our results to the original Abell/ACO catalog (Abell et al. 1989) based on the most recent parameter measurements for its clusters (e.g., Chow-Martinez et al. 2014). Also, we compared the detected systems against the central galaxy position provided by the Brightest Cluster Galaxy catalog (Lauer et al. 2014, hereafter L14). Regarding catalogs based on the SDSS spectroscopic sample we compared with the C4 cluster catalog (Miller et al. 2005), based on the SDSS-DR2. For less rich clusters and groups we compared our systems with the Multi-scale Probability Mapping clusters/groups catalog (MSPM, Smith et al. 2012) and the Tempel et al. (2012) catalog (hereafter T11), carried over the SDSS-DR7 and -DR8 respectively. For the comparisons we used all systems detected by GSyF down to a richness of five galaxies.

Using the tolerance cylinder described above, 19 of the 37 Abell/ACO clusters inside the MSCC 310 box were detected as systems of richness above five galaxies with our method (51\%), while the equivalent number was $26(76 \%)$ for the 34 clusters in $\mathrm{C} 4$. There are 11 clusters in the L14 catalog embedded in the volume and 8 (73\%) of them have GSyF counterparts. However, by increasing the aperture to $2 \mathrm{~h}_{70}^{-1} \mathrm{Mpc}$, we increased the detection of Abell clusters to 29/37 (78\%), C4 clusters to 33/34 (97\%) and the L14 catalog to $100 \%$; see Table 6. The increase of $20-30 \%$ in cluster matches caused by using a larger aperture size can be related to the fact that the mean separation of member galaxies increases for lower richness systems, and the 

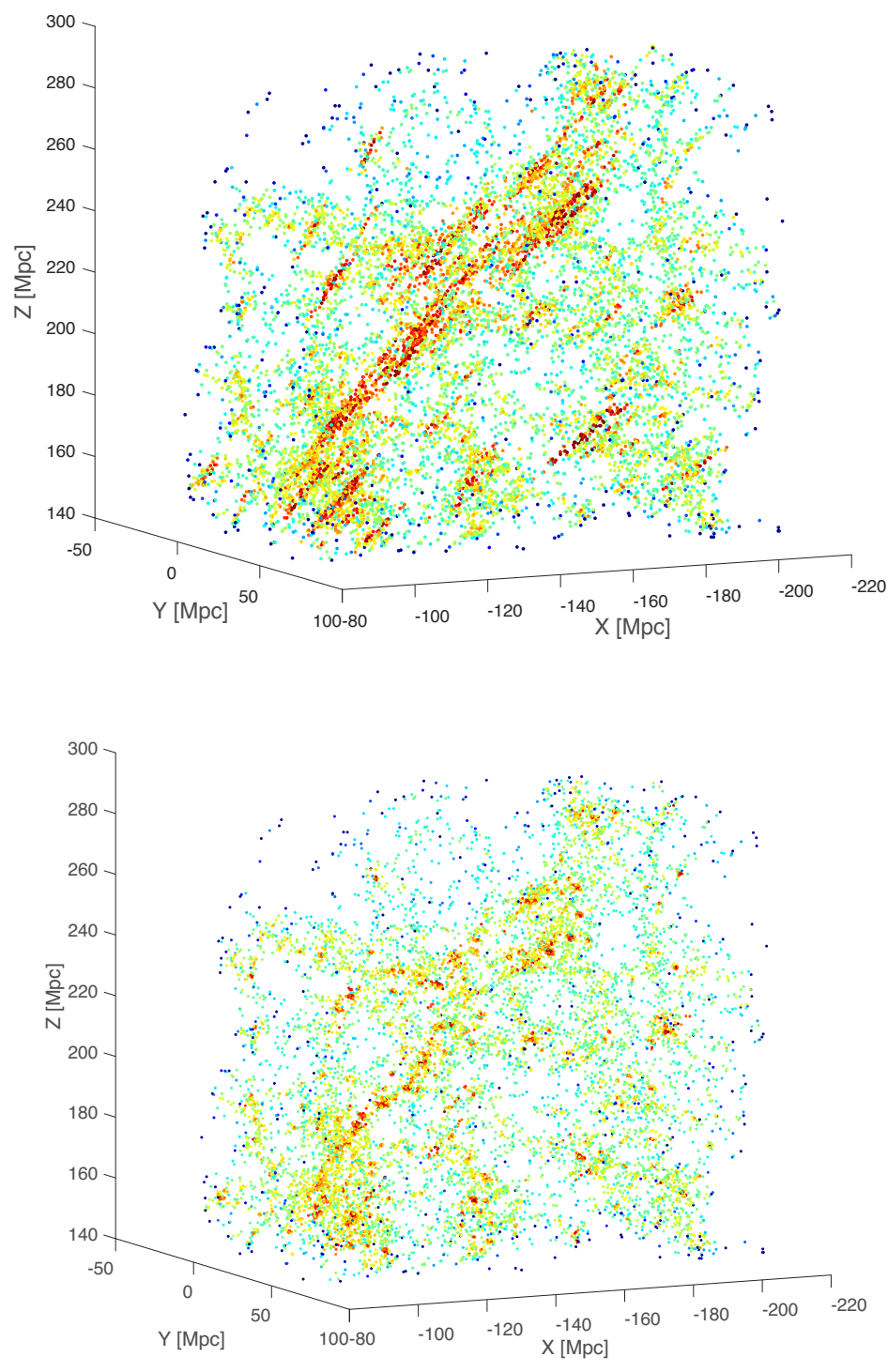

Fig. 5. Three-dimensional distribution of galaxies for the MSCC 310 supercluster volume. Top: Galaxy positions before the application of the FoG correction. Bottom: Galaxy positions after correction for FoG effects. The colors represent density as calculated from 3D VT. The highest density is represented in red while lower densities are represented in green to blue.

determination of the cluster center is then subject to this separation; see Table 6. For example, A1452 and A1507 B have a GSyF counterpart located at $\sim 1.5 \mathrm{~h}_{70}^{-1} \mathrm{Mpc}$ projected distance and $\Delta \sigma_{v}$ of $\sim 630 \mathrm{~km} \mathrm{~s}^{-1}$ and $120 \mathrm{~km} \mathrm{~s}^{-1}$ respectively (see Table 4 , systems No. 12 and 14), while their C4 counterparts are 0.7 and $0.4 \mathrm{~h}_{70}^{-1} \mathrm{Mpc}$ away respectively.

For what concerns the less massive systems, there are 315 groups detected by T11 and 213 groups listed in the MSPM catalog with richness larger than or equal to five galaxies for the MSCC 310 volume. Our algorithm detected systems that correspond to $61 \%(79 \%)$ of the T11 groups and 67\% (78\%) of the MSPM groups, within an aperture of $1 \mathrm{~h}_{70}^{-1} \mathrm{Mpc}\left(2 \mathrm{~h}_{70}^{-1} \mathrm{Mpc}\right)$; see Table 6. This is acceptable for our purposes because we have constructed GSyF to find the clusters that present the FoG effect, although we can clearly go farther towards poorer systems with GSyF.

The region of the UMa supercluster was studied by Krause et al. (2013). These authors identified 31 galaxy systems

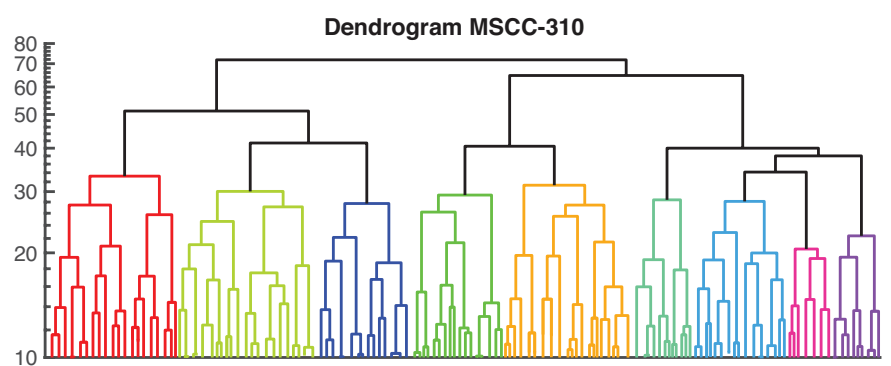

Filaments MSCC-310

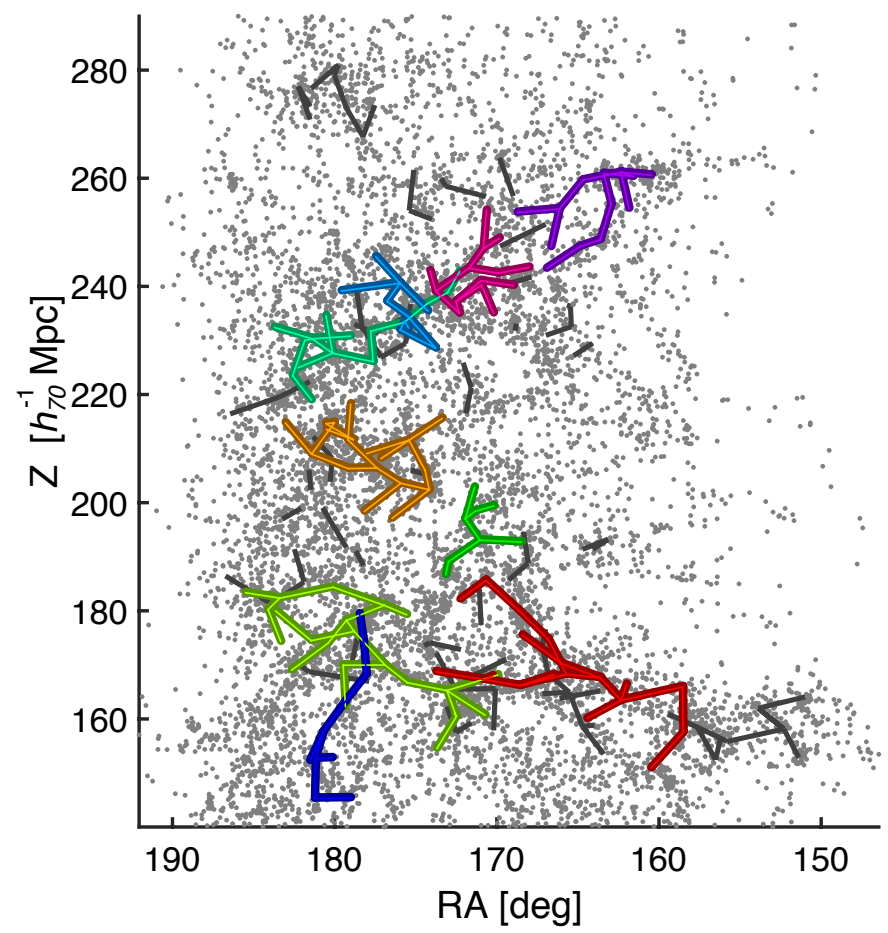

Fig. 6. Results of the GFiF algorithm for the MSCC 310 supercluster volume. Upper panel: dendrogram with the nine detected filaments represented by different colors. The $y$ axis of the dendrogram plot indicates the distance at each level of the tree. Lower panel: $\mathrm{RA} \times Z$ distribution, where SDSS galaxies are represented by gray points and filaments are represented by lines according to the colors in the upper panel. Tendrils are represented by gray lines.

in the MSCC 310 area, each with between 15 and 94 galaxies. We found that our GSyF systems match with 24 (77\%) of these clusters within an aperture of $3 \mathrm{~h}_{70}^{-1} \mathrm{Mpc}$, of which 10 are Abell clusters.

The systems detected in the main portion of the MSCC 310 supercluster are depicted on a sky projected distribution in Fig. 7 (top panel) and are represented by black circles with radii equal to the measured virial radius. The system positions from the Abell, C4, L14, MSPM, and T11 catalogs are depicted as red, pink, cyan, blue, and green points, respectively. We also observe that the system membership number detected by GSyF is in agreement, for most of the cases, with the number of members for the same systems detected by T11, C4, and MSPM (see Fig. 8). Qualitatively one can observe in this figure that the richness from T11 is in better agreement with our measurements, while MSPM estimates a richness slightly lower than both ours and T11.

A similar analysis can be done for the other superclusters in our sample. For example, for the Coma supercluster (MSCC 295, Fig. 7, bottom panel), the GSyF algorithm detected 115 systems 
Table 5. Main properties of the filaments extracted through GFiF for the supercluster MSCC 310.

\begin{tabular}{|c|c|c|c|c|c|c|c|c|c|c|}
\hline \multirow{2}{*}{$\begin{array}{l}\text { Fil. } \\
\text { ID } \\
(1)\end{array}$} & \multirow{2}{*}{$\begin{array}{r}N_{\text {sfil }} \\
\text { systems } \\
(2) \\
\end{array}$} & \multirow{2}{*}{$\begin{array}{c}N_{\text {gfil }} \\
\text { gals. } \\
\text { (3) }\end{array}$} & \multicolumn{3}{|c|}{ Redshift } & \multirow{2}{*}{$\begin{array}{l}d_{\text {fil }} \\
{\left[\mathrm{h}_{70}^{3} \mathrm{Mpc}^{-3}\right]} \\
(7)\end{array}$} & \multirow{2}{*}{$\begin{array}{c}R_{\mathrm{fil}} \\
{\left[\mathrm{h}_{70}^{-1} \mathrm{Mpc}\right]} \\
\quad(8)\end{array}$} & \multicolumn{2}{|c|}{$N_{\text {nod }}$} & \multirow{2}{*}{$\begin{array}{c}\ell_{\text {fil }} \\
{\left[\mathrm{h}_{70}^{-1} \mathrm{Mpc}\right]} \\
(11)\end{array}$} \\
\hline & & & $\begin{array}{l}\text { [mean, } \\
\text { (4) }\end{array}$ & $\begin{array}{c}\min , \\
(5)\end{array}$ & $\begin{array}{c}\max ] \\
(6)\end{array}$ & & & $\begin{array}{r}\text { Filament } \\
(9) \\
\end{array}$ & $\begin{array}{r}\text { Skeleton } \\
(10) \\
\end{array}$ & \\
\hline MSCC 310-F1 & 10 & 499 & 0.0609 & 0.0518 & 0.0689 & 0.4180 & 2.81 & 18 & 11 & 61.6 \\
\hline MSCC 310-F2 & 8 & 523 & 0.0502 & 0.0443 & 0.0588 & 0.5210 & 2.84 & 22 & 11 & 51.8 \\
\hline MSCC $310-F 3$ & 7 & 407 & 0.0481 & 0.0427 & 0.0528 & 0.3486 & 2.50 & 19 & 10 & 49.0 \\
\hline MSCC 310-F4 & 8 & 313 & 0.0656 & 0.0585 & 0.0710 & 0.4263 & 2.46 & 14 & 10 & 59.0 \\
\hline MSCC 310-F5 & 7 & 325 & 0.0700 & 0.0642 & 0.0774 & 0.5303 & 2.60 & 13 & 7 & 47.6 \\
\hline MSCC 310-F6 & 6 & 243 & 0.0725 & 0.0651 & 0.0791 & 0.2986 & 1.97 & 12 & 9 & 39.3 \\
\hline MSCC 310-F7 & 4 & 219 & 0.0551 & 0.0479 & 0.0619 & 0.4062 & 2.76 & 7 & 5 & 20.7 \\
\hline MSCC 310-F8 & 4 & 164 & 0.0546 & 0.0485 & 0.0617 & 0.2118 & 2.22 & 9 & 7 & 33.7 \\
\hline MSCC 310-F9 & 4 & 124 & 0.0464 & 0.0437 & 0.0528 & 0.1464 & 1.13 & 9 & 6 & 17.9 \\
\hline
\end{tabular}

Notes. The columns in the table are as follows: (1) sequential number assigned to the filament; (2) number of systems detected by GSyF and linked by the filament; (3) number of galaxies attributed to the filament; (4) mean redshift of the filament; (5) minimum redshift of the filament; (6) maximum redshift of the filament; (7) mean number density inside the filament; (8) mean transversal radius of the filament measured at 10 $\times d$; (9) number of nodes that constitute the filament; (10) number of central skeleton nodes in the filament; (11) length of the filament skeleton.

Table 6. GSyF systems detected by other catalogs for the MSCC 310 supercluster.

\begin{tabular}{|c|c|c|c|c|c|c|}
\hline \multirow[t]{2}{*}{ Other catalog } & \multirow{2}{*}{$\begin{array}{l}\text { Number } \\
N_{\text {mem }}\end{array}$} & \multirow{2}{*}{$\begin{array}{l}\text { Fraction } \\
>5\end{array}$} & \multirow{2}{*}{$\begin{array}{l}\text { Number } \\
N_{\text {mem }} \\
\end{array}$} & \multirow{2}{*}{$\begin{array}{l}\text { Fraction } \\
>10\end{array}$} & \multicolumn{2}{|c|}{ Separation } \\
\hline & & & & & $\mathrm{h}_{70}^{-1} \mathrm{Mpc}$ & $\Delta \sigma_{v}$ \\
\hline \multicolumn{7}{|c|}{ Aperture $=1 \mathrm{~h}_{70}^{-1} \mathrm{Mpc}$} \\
\hline Abell & $19 / 37$ & $51 \%$ & $17 / 37$ & $46 \%$ & 0.45 & 295 \\
\hline C4 & $26 / 34$ & $76 \%$ & $24 / 34$ & $71 \%$ & 0.43 & 100 \\
\hline L14 & $8 / 11$ & $73 \%$ & $8 / 11$ & $73 \%$ & 0.50 & 340 \\
\hline T11 & $192 / 315$ & $61 \%$ & $73 / 105$ & $70 \%$ & 0.33 & 230 \\
\hline MSPM & $142 / 213$ & $67 \%$ & $63 / 79$ & $80 \%$ & 0.34 & 145 \\
\hline \multicolumn{7}{|c|}{ Aperture $=2 \mathrm{~h}_{70}^{-1} \mathrm{Mpc}$} \\
\hline Abell & $29 / 37$ & $78 \%$ & $24 / 37$ & $65 \%$ & 0.82 & 300 \\
\hline C4 & $33 / 34$ & $97 \%$ & $32 / 34$ & $94 \%$ & 0.77 & 166 \\
\hline L14 & $11 / 11$ & $100 \%$ & $11 / 11$ & $100 \%$ & 0.80 & 430 \\
\hline T11 & 249/315 & $79 \%$ & $85 / 105$ & $81 \%$ & 0.64 & 320 \\
\hline MSPM & $167 / 213$ & $78 \%$ & $68 / 79$ & $86 \%$ & 0.55 & 193 \\
\hline
\end{tabular}

in total. Of these, we found that A1656, the richest one, is composed of 579 galaxies. The estimated virial radius and mass are $1.96 \mathrm{~h}_{70}^{-1} \mathrm{Mpc}$ and $7.7 \times 10^{14} M_{\odot}$, respectively. The second-richest cluster, A1367, has 243 galaxies, while its radius and mass are $1.73 \mathrm{~h}_{70}^{-1} \mathrm{Mpc}$ and $5.3 \times 10^{14} M_{\odot}$, respectively. These estimations are in good agreement with those measured by Rines et al. (2003). The complete catalog of systems for each volume is available online ${ }^{2}$.

\subsection{Checking the filament skeletons}

We compared the filaments obtained using the GFiF algorithm for the MSCC 310 volume with those presented by Tempel et al. (2014, hereafter T14) as extracted from their Table 2. We transformed the T14 filament positions in survey coordinates (see T14, Eq. (1)) to our rectangular space and cosmology. There are about 630 T14 filaments that lie in the sampled volume of the MSCC 310 supercluster. These filaments have a mean length of $9 \mathrm{~h}_{70}^{-1} \mathrm{Mpc}$ while the largest one has a length of $48 \mathrm{~h}_{70}^{-1} \mathrm{Mpc}$. As a comparison, the filament skeletons detected by GFiF have a mean length of $42 \mathrm{~h}_{70}^{-1} \mathrm{Mpc}$ and the largest one has a length of $62 \mathrm{~h}_{70}^{-1} \mathrm{Mpc}$. We found a $40 \%$ match between our detected fila-

\footnotetext{
2 https://gitlab.com/iris.santiagob89/LSS_structures
}

ments and T14 and an $80 \%$ match with our isolated bridges and tendrils. The mean difference between the medial axis of the T14 filaments matching the nearest filament or tendril detected by us is $\sim 1.5 \mathrm{~h}_{70}^{-1} \mathrm{Mpc}$. In Fig. 9, T14 filaments are represented by a sequence of points forming a line. The calculated separation was taken to be the distance from the T14 filament points to the edges of our filaments. Our filaments are depicted over T14 filaments in this figure. As can be seen, GFiF detects the most prominent (dense) filaments among the ones in T14.

\subsection{Comparison with KDE density maps}

In order to validate the results from GSyF and GFiF algorithms and specifically to corroborate the densities, we performed an independent analysis of the galaxies in the MSCC 310 volume using the KDE method, as described in Sect. 3.6. A quantitative comparison in the space between the 3D KDE and the skeleton structures is left for upcoming works. Here, we restricted our analysis to $2 \mathrm{D}$ projections (density maps) of the $3 \mathrm{D} \mathrm{KDE}$ $(X Y, X Z, Y Z)$. For this analysis we used kernels of $1 \Sigma$ in size (see Sect. 3.6). Since each kernel is created based on the VT cell, we used $d_{\text {bas }}$ as a baseline density. We selected those regions for which $d_{\mathrm{KDE}}>d_{\text {bas }}$ in the RA $\times$ Dec projected density map. Subsequently, we compared the position of the density peak of 

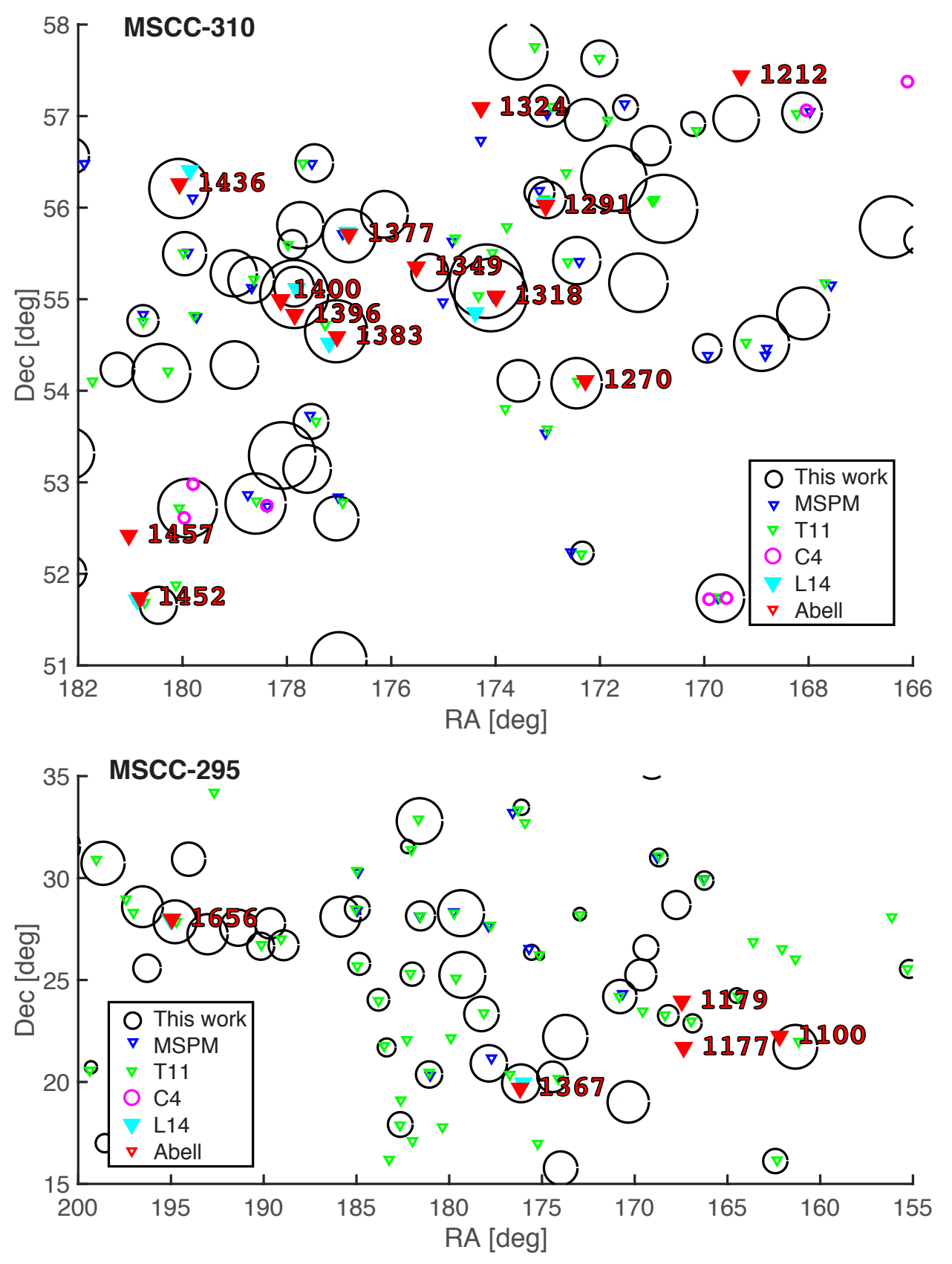

Fig. 7. Projected distribution in the sky of systems detected by GSyF. Top: systems detected for the MSCC 310 (UMa supercluster). Bottom: systems detected for the MSCC 295 (Coma supercluster). The system radii are shown as circles of $r=$ $R_{\mathrm{vir}}$. For comparison, the positions of systems reported by MSPM, T11, C4, L14, and Abell catalogs are depicted by color points: blue, green, pink, cyan, and red, respectively.

each region against the centroids of the $122 \mathrm{GSyF}$ systems. We found that $93 \mathrm{GSyF}$ systems with $N_{j} \geq 10(76 \%)$ match density peaks above $3 d_{\text {bas }}$. The remaining 29 GSyF systems (24\%) are identified with density peaks in the range $(1-3) d_{\text {bas }}$. Moreover, we observe that the filament edges connect these density peaks forming chains of overdensity regions. Figure 10 (top panel) shows the systems detected by GSyF represented by circles of $r=R_{\text {vir }}$ over the galaxy density distribution as measured using $\mathrm{KDE}$ in a RA $\times$ Dec projection. The bottom panel of Fig. 10 shows the filaments overlaid on the KDE density map for the MSCC 310 volume. The density maps are set in terms of the mean number density.

\section{Filament properties}

\subsection{Main properties of the filaments}

In a similar way as described for MSCC 310, we applied the GFiF algorithm to the 46 superclusters of our sample, detect- ing a total of 144 filaments in 40 superclusters which are listed in Table 7. This table also lists the parameters used or measured by GFiF. The process of filtering the connections can be followed through columns 5-10. The list of detected filaments for all studied supercluster volumes can be consulted in Table A.1, in the same format as in Table 5. The MSCC 75, MSCC 76, MSCC 264, MSCC 441, MSCC 579 and MSCC 586 superclusters have not been evaluated with GFiF due to the sparseness of the SDSS coverage in these sky areas.

The filament skeletons detected by GFiF have lengths of between 9 and $130 \mathrm{~h}_{70}^{-1} \mathrm{Mpc}$. Figure 11 depicts the length distribution for all the detected filaments. The distribution shows that the majority of the structure lengths range from 10 to $40 \mathrm{~h}_{70}^{-1} \mathrm{Mpc}$. There are two structures longer than $100 \mathrm{~h}_{70}^{-1} \mathrm{Mpc}$. The first is the filament of $130 \mathrm{~h}_{70}^{-1} \mathrm{Mpc}$ in length located in MSCC 323, which contains the Abell clusters A1449B and $\mathrm{A} 1532 \mathrm{~A}$, and the second, of $105 \mathrm{~h}_{70}^{-1} \mathrm{Mpc}$, is located in MSCC 335, which contains A1478 A, A1480 B, and A1486 A. 


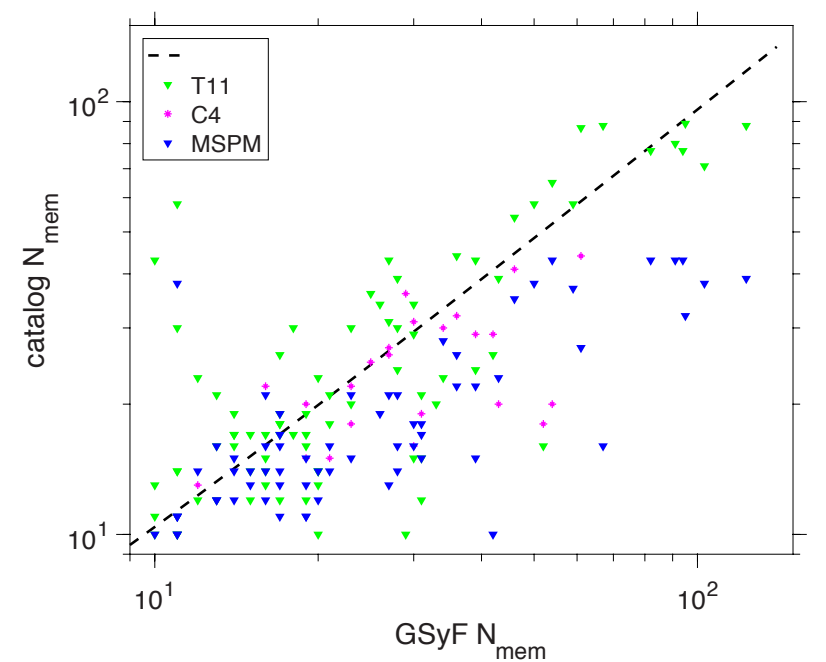

Fig. 8. Comparison of MSCC 310 supercluster GSyF system richness against the richness measured by other catalogs for the matching systems. Symbol colors are the same as the ones in Fig. 7. The dashed line represents the identity.

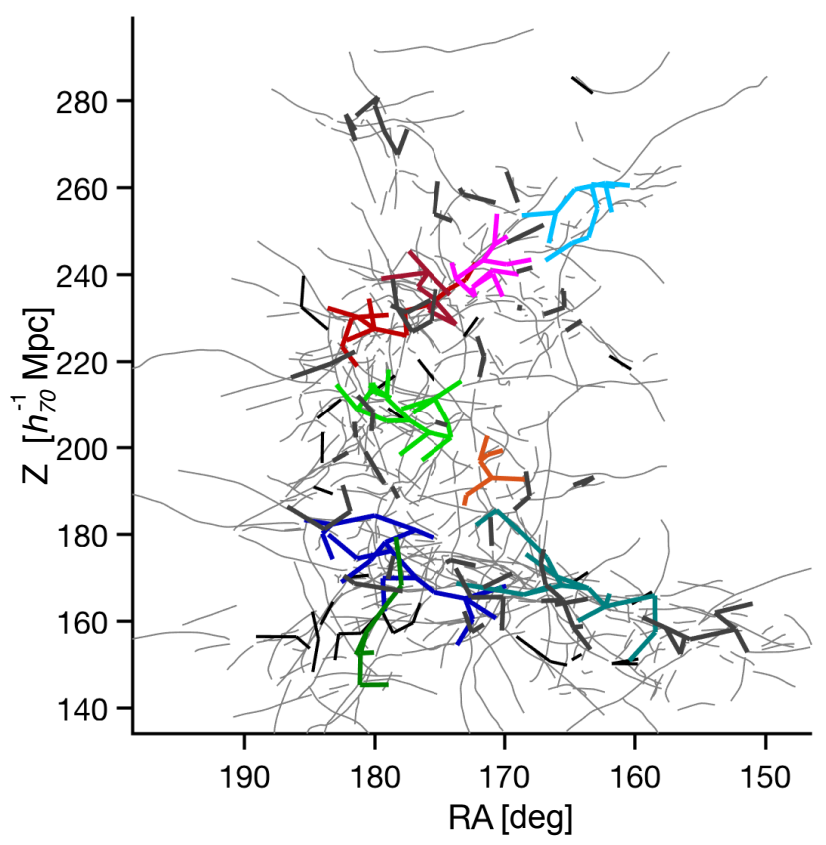

Fig. 9. Comparison of GFiF filaments for the MSCC 310 supercluster to the T14 filaments in the same region for the SDSS-DR8. Gray lines are T14 filaments. Colored lines depict filaments identified in this work.

Excluding these two particular cases, we observe that the mean length of the filaments is about $37 \mathrm{~h}_{70}^{-1} \mathrm{Mpc}$ while the median corresponds to $29 \mathrm{~h}_{70}^{-1} \mathrm{Mpc}$.

\subsection{Distribution of galaxies along the filaments}

In order to evaluate the environment within the filaments, we extracted longitudinal profiles of number density. Figure 12 shows the longitudinal distribution of galaxies for all bridges, from one extreme to the other (ending systems), in the supercluster MSCC 310 . We observe that the density of galaxies is higher near the ends of the bridges, as expected, and decreases through the midpoint between systems. We then proceeded to
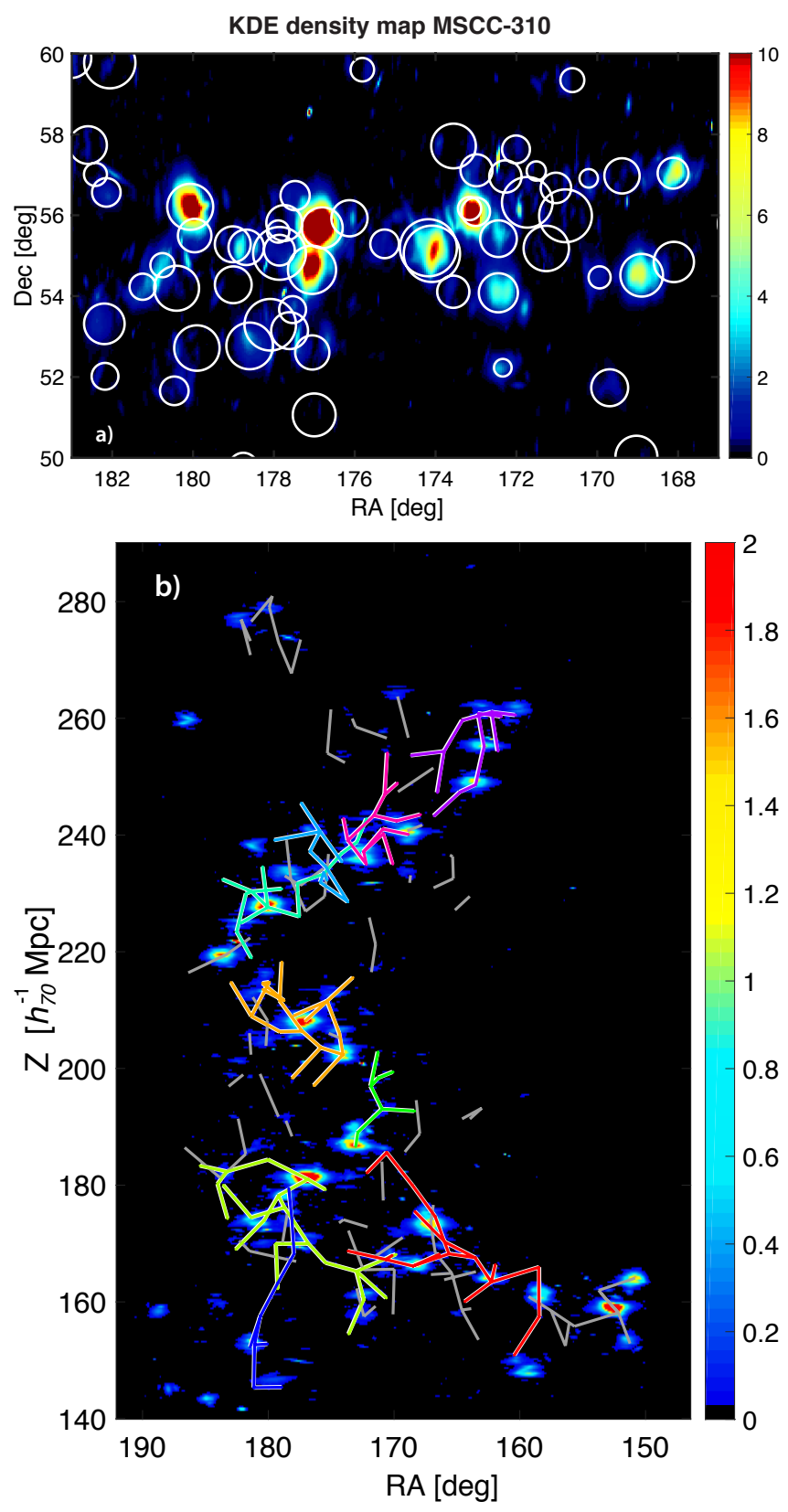

Fig. 10. Top: $\mathrm{RA} \times$ Dec projected density map as measured from $3 \mathrm{D}$ $\mathrm{KDE}$ with $1 \Sigma$ in terms of the density contrast. The GSyF systems are represented by white circles with radius scaled to the estimated $R_{\mathrm{vir}}$. Bottom: $\mathrm{RA} \times \mathrm{Z}$ projection. The filaments detected in this work are overlaid in color. Density is represented following the color scale displayed on the right, where denser regions are redder and less dense zones are bluer.

extract density profiles for bridges, from the systems to the midpoint, by counting the galaxies that lie within a cylinder of radius $1 \mathrm{~h}_{70}^{-1} \mathrm{Mpc}$ with the medial skeleton set by the bridge skeleton. The galaxies are counted in slices of size $\Delta d=0.5 \mathrm{~h}_{70}^{-1} \mathrm{Mpc}$ along the skeleton axes. We also calculated the longitudinal profiles after excluding the galaxies belonging to systems (considered at $1.5 R_{\mathrm{vir}}$ ) from their bridges in order to determine pure filament profiles. These profiles allow us to evaluate the mean density contrast of the filaments as compared with the background density. In Fig. 13 we show the longitudinal number density profile for all filaments detected in our sample. The stacked longitudinal profile including galaxies in systems is depicted by 
Table 7. Summary of the properties of the filaments detected by GFiF for the superclusters in Table 1.

\begin{tabular}{|c|c|c|c|c|c|c|c|c|c|c|c|c|c|c|c|c|}
\hline \multirow{2}{*}{$\begin{array}{l}\text { MSCC } \\
\text { ID } \\
\text { (1) }\end{array}$} & \multirow{2}{*}{$\begin{array}{l}f \\
\text { (2) } \\
\end{array}$} & \multirow{2}{*}{$\begin{array}{l}N_{\text {cut }} \\
\\
(3) \\
\end{array}$} & \multirow{2}{*}{$\begin{array}{r}D_{\max } \\
\\
\quad(4) \\
\end{array}$} & \multirow{2}{*}{$\begin{array}{r}N_{\text {edges }} \\
\\
\quad(5) \\
\end{array}$} & \multirow{2}{*}{$\begin{array}{r}N_{\text {links }} \\
\\
(6) \\
\end{array}$} & \multirow{2}{*}{$\begin{array}{r}N_{\text {trees }} \\
\\
(7) \\
\end{array}$} & \multirow{2}{*}{$\begin{array}{r}N_{\text {ske }} \\
\\
(8) \\
\end{array}$} & \multirow{2}{*}{$\begin{array}{l}N_{\text {brid }} \\
\\
(9) \\
\end{array}$} & \multirow{2}{*}{$\begin{array}{l}N_{\text {tend }} \\
(10) \\
\end{array}$} & \multirow{2}{*}{$\begin{array}{l}N_{\min } \\
(11) \\
\end{array}$} & \multicolumn{3}{|c|}{ Fraction (\%) of systems in } & \multirow{2}{*}{$\begin{array}{l}N_{\text {gfil }} \\
(15) \\
\end{array}$} & \multicolumn{2}{|c|}{ Filling factor $(\%)$} \\
\hline & & & & & & & & & & & $\begin{array}{r}\text { Filaments } \\
N_{\text {sfil }} / N_{\text {sys }} \\
(12)\end{array}$ & $\begin{array}{r}\text { Bridges } \\
N_{\text {spair }} / N_{\text {sys }} \\
(13) \\
\end{array}$ & $\begin{array}{r}\text { Isolated } \\
N_{\text {sout }} / N_{\text {sys }} \\
(14) \\
\end{array}$ & & $V_{\text {fil }} / V$ & $\begin{array}{r}N_{\text {gfil }} / N \\
(17) \\
\end{array}$ \\
\hline 55 & 10 & 81 & 6 & 7 & 6 & 2 & 2 & 0 & 0 & 10 & 63.6 & 0.0 & 36.4 & 121 & 0.2 & 14.9 \\
\hline 72 & 16 & 121 & 8 & 29 & 28 & 6 & 4 & 1 & 1 & 8 & 56.7 & 6.7 & 36.7 & 829 & 1.0 & 42.7 \\
\hline 175 & 29 & 86 & 14 & 36 & 29 & 4 & 4 & 0 & 0 & 6 & 57.7 & 0.0 & 42.3 & 507 & 0.9 & 20.2 \\
\hline 184 & 27 & 78 & 15 & 29 & 13 & 2 & 2 & 0 & 0 & 6 & 57.1 & 0.0 & 42.9 & 218 & 0.6 & 10.4 \\
\hline 211 & 16 & 93 & 12 & 29 & 22 & 2 & 1 & 0 & 1 & 5 & 100.0 & 0.0 & 0.0 & 233 & 1.0 & 15.7 \\
\hline 219 & 27 & 71 & 17 & 65 & 36 & 3 & 2 & 2 & -1 & 5 & 72.2 & 22.2 & 5.6 & 279 & 1.1 & 14.6 \\
\hline 222 & 15 & 124 & 16 & 84 & 43 & 5 & 2 & 0 & 3 & 4 & 44.4 & 0.0 & 55.6 & 97 & 0.2 & 5.2 \\
\hline 223 & 15 & 52 & 18 & 31 & 19 & 2 & 1 & 0 & 1 & 4 & 75.0 & 0.0 & 25.0 & 11 & 0.1 & 1.4 \\
\hline 229 & 16 & 116 & 19 & 93 & 44 & 8 & 1 & 1 & 6 & 4 & 37.5 & 25.0 & 37.5 & 32 & 0.1 & 1.7 \\
\hline 236 & 32 & 270 & 9 & 124 & 94 & 11 & 7 & 2 & 2 & 18 & 55.3 & 8.5 & 36.2 & 1600 & 0.8 & 18.5 \\
\hline 238 & 26 & 320 & 18 & 215 & 115 & 20 & 6 & 3 & 11 & 6 & 39.0 & 10.2 & 50.8 & 544 & 0.4 & 6.5 \\
\hline 248 & 21 & 60 & 20 & 47 & 20 & 3 & 1 & 0 & 2 & 5 & 50.0 & 0.0 & 50.0 & 159 & 0.9 & 12.6 \\
\hline 266 & 22 & 44 & 16 & 20 & 12 & 1 & 1 & 0 & 0 & 5 & 71.4 & 0.0 & 28.6 & 132 & 0.7 & 13.8 \\
\hline 272 & 16 & 86 & 6 & 14 & 14 & 3 & 2 & 0 & 1 & 8 & 90.0 & 0.0 & 10.0 & 453 & 0.9 & 32.8 \\
\hline 277 & 15 & 183 & 11 & 77 & 62 & 6 & 2 & 0 & 4 & 5 & 62.5 & 0.0 & 37.5 & 611 & 1.0 & 22.2 \\
\hline 278 & 16 & 495 & 7 & 378 & 256 & 20 & 5 & 1 & 14 & 19 & 58.1 & 4.7 & 37.2 & 2144 & 1.6 & 27.1 \\
\hline 283 & 23 & 101 & 16 & 32 & 21 & 5 & 3 & 0 & 2 & 4 & 44.4 & 0.0 & 55.6 & 263 & 0.5 & 11.3 \\
\hline 295 & 14 & 1022 & 5 & 478 & 398 & 38 & 4 & 5 & 29 & 26 & 43.1 & 19.6 & 37.3 & 2992 & 1.0 & 20.9 \\
\hline 310 & 16 & 768 & 8 & 334 & 273 & 34 & 9 & 7 & 18 & 10 & 52.7 & 12.7 & 34.5 & 2817 & 1.0 & 22.9 \\
\hline 311 & 25 & 211 & 10 & 49 & 37 & 5 & 4 & 0 & 1 & 7 & 43.6 & 0.0 & 56.4 & 1118 & 0.8 & 21.2 \\
\hline 314 & 14 & 40 & 8 & 13 & 7 & 2 & 2 & 0 & 0 & 7 & 50.0 & 0.0 & 50.0 & 112 & 0.7 & 20.1 \\
\hline 317 & 15 & 56 & 17 & 56 & 31 & 3 & 2 & 1 & 0 & 5 & 61.5 & 15.4 & 23.1 & 63 & 0.5 & 7.5 \\
\hline 323 & 22 & 151 & 16 & 46 & 29 & 5 & 2 & 2 & 1 & 4 & 26.3 & 10.5 & 63.2 & 239 & 0.5 & 7.2 \\
\hline 333 & 19 & 104 & 11 & 39 & 28 & 6 & 3 & 1 & 2 & 7 & 45.8 & 8.3 & 45.8 & 282 & 0.6 & 14.3 \\
\hline 335 & 23 & 135 & 13 & 108 & 65 & 8 & 3 & 0 & 5 & 8 & 52.9 & 0.0 & 47.1 & 478 & 1.1 & 15.4 \\
\hline 343 & 11 & 244 & 7 & 51 & 33 & 5 & 3 & 1 & 1 & 7 & 34.5 & 6.9 & 58.6 & 335 & 0.4 & 12.5 \\
\hline 360 & 38 & 58 & 20 & 54 & 23 & 4 & 3 & 0 & 1 & 6 & 47.6 & 0.0 & 52.4 & 218 & 0.8 & 9.9 \\
\hline 386 & 9 & 362 & 7 & 165 & 120 & 20 & 4 & 2 & 14 & 9 & 60.6 & 12.1 & 27.3 & 636 & 0.7 & 19.5 \\
\hline 407 & 16 & 70 & 18 & 49 & 20 & 3 & 1 & 0 & 2 & 4 & 50.0 & 0.0 & 50.0 & 101 & 0.7 & 9.0 \\
\hline 414 & 9 & 1211 & 6 & 462 & 386 & 47 & 15 & 12 & 20 & 9 & 42.4 & 16.7 & 41.0 & 2232 & 0.8 & 20.5 \\
\hline 419 & 15 & 115 & 11 & 39 & 22 & 5 & 3 & 3 & -1 & 5 & 28.6 & 17.1 & 54.3 & 254 & 0.3 & 14.7 \\
\hline 422 & 18 & 59 & 19 & 35 & 10 & 1 & 1 & 0 & 0 & 4 & 37.5 & 0.0 & 62.5 & 11 & 0.0 & 1.0 \\
\hline 430 & 20 & 80 & 12 & 35 & 22 & 5 & 4 & 1 & 0 & 6 & 58.3 & 8.3 & 33.3 & 186 & 0.5 & 11.6 \\
\hline 440 & 15 & 229 & 10 & 56 & 36 & 8 & 1 & 2 & 5 & 5 & 20.7 & 13.8 & 65.5 & 184 & 0.2 & 5.3 \\
\hline 454 & 15 & 380 & 6 & 164 & 117 & 15 & 5 & 4 & 6 & 13 & 45.9 & 13.1 & 41.0 & 1516 & 1.2 & 26.6 \\
\hline 457 & 21 & 194 & 9 & 80 & 68 & 7 & 6 & 0 & 1 & 8 & 66.7 & 0.0 & 33.3 & 1525 & 1.9 & 37.5 \\
\hline 460 & 22 & 159 & 14 & 102 & 65 & 6 & 4 & 1 & 1 & 5 & 65.9 & 4.5 & 29.5 & 895 & 1.4 & 25.6 \\
\hline 463 & 16 & 529 & 8 & 230 & 183 & 27 & 11 & 8 & 8 & 8 & 47.2 & 12.8 & 40.0 & 2228 & 1.1 & 26.3 \\
\hline 474 & 15 & 495 & 5 & 245 & 209 & 20 & 7 & 3 & 10 & 16 & 48.0 & 12.0 & 40.0 & 1918 & 0.9 & 25.8 \\
\hline 484 & 22 & 60 & 16 & 13 & 9 & 1 & 1 & 0 & 0 & 4 & 44.4 & 0.0 & 55.6 & 109 & 0.6 & 8.3 \\
\hline
\end{tabular}

Notes. The columns in the table are as follows: (1) ID of the supercluster in MSCC; (2) segmentation parameter $f$; (3) number of detected HC groups in the supercluster box; (4) linking length $\left(D_{\max }\right)$ used to connect HC groups; (5) number of detected edges; (6) number of filtered links; (7) number of trees detected after applying MST; (8) final number of filaments, $N_{\text {ske }}$; (9) number of isolated bridges, $N_{\text {brid }}$; (10) number of tendrils, $N_{\text {tend }}$; (11) minimum richness we considered for GSyF systems to be taken as ends of the bridges; (12) fraction of these systems included in the GFiF filaments; (13) fraction of these systems included in isolated bridges; (14) fraction of these systems not connected by bridges; (15) number of galaxies hosted in the GFiF filaments, $N_{\text {gfil }} ;(16)$ filling factor calculated as $V_{\text {fil }} / V$; (17) filling factor calculated as $N_{\text {gfil }} / N$.

a blue line. The dispersion about the stacked profile is represented by a blue shaded area. The pure profiles (excluding the galaxies of the systems) is represented by the red line, and its corresponding dispersion by a red shaded area. As can be seen, the mean density contrast along the filament is $\sim 10$, that is, the filament is about ten times denser than background.

\subsection{Transversal density profiles}

For the calculation of transversal density profiles, we excluded the galaxies located in systems and within a radius of $1.5 R_{\mathrm{vir}}$.
The density profile is calculated as described in Sect. 3.7. The cylinder radius $R_{\text {cy }}$ was set from 0 up to $10 \mathrm{~h}_{70}^{-1} \mathrm{Mpc}$ in steps of $\Delta R_{\text {cy }}=0.5 \mathrm{~h}_{70}^{-1} \mathrm{Mpc}$.

We computed the galaxy number density profile for filaments in two ways. First we counted the number of galaxies within concentric cylinders and divided them by the volume within the cylinders. We refer to this as the local number density profile. For the second, we employed the number densities calculated using the VT $d_{i}$, as described in Sect. 3.1. We then measured the mean VT number density within concentric cylinders. The local number density and VT number density profiles are scaled 


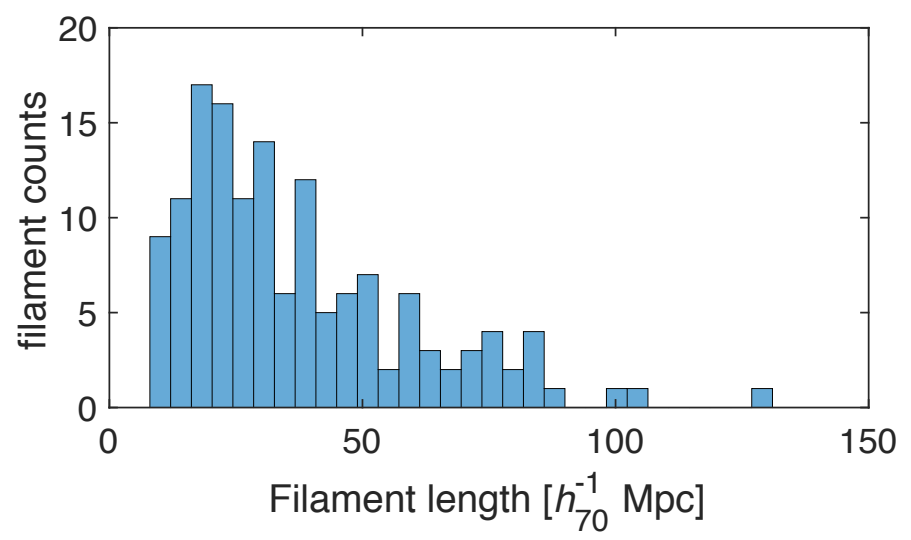

Fig. 11. Distribution of filament skeleton length for the 144 filaments detected by GFiF. The length used corresponds to the longest path between the systems at the extremity of the filament. See Table A.1.

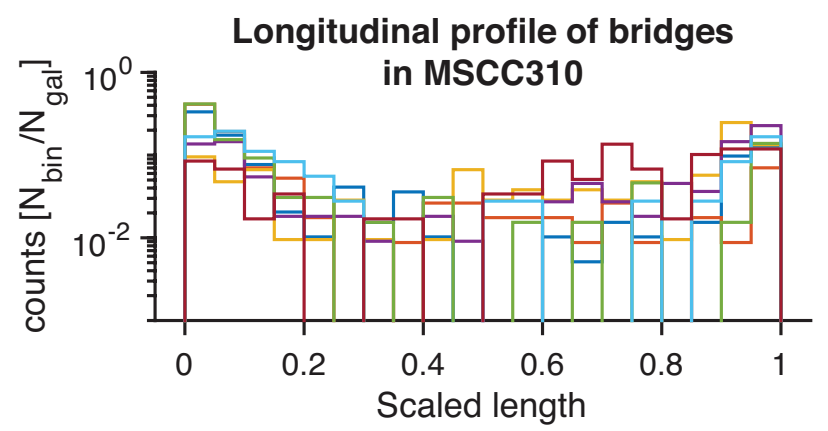

Fig. 12. Distribution of galaxies along bridges connecting pairs of systems for the nine MSCC 310 filaments. All bridges are scaled to length 1.0 .

in density contrast and stacked together. Figure 14 shows the stacked profile for all filaments detected by GFiF, for local densities (top panel) and VT densities (bottom panel), respectively. The first aspect to note is that local number density profiles are smoother, although in general both mean profiles are similar. We can observe in both local and VT density profiles that overdensity extends up to $5 \mathrm{~h}_{70}^{-1} \mathrm{Mpc}$. At about $3 \mathrm{~h}_{70}^{-1} \mathrm{Mpc}$, the overdensity reaches a value of around 3 , while the typical characteristic density contrast of 10 is reached closer to $2 \mathrm{~h}_{70}^{-1} \mathrm{Mpc}$.

Finally, we used the density profiles to estimate the mean radius of the filaments $R_{\text {fil }}$. This was achieved by considering the intersection point at which the local density profile crosses the $10 \times d$ line, as indicated in Fig. 14 by the black solid line. The mean radius as well as the mean density of each filament is noted in Table A.1.

Figure 15 (top panel) presents the radii distribution for all the filaments. The filament radii range from 0.6 to $4.5 \mathrm{~h}_{70}^{-1} \mathrm{Mpc}$ with a mean value of $2.4 \mathrm{~h}_{70}^{-1} \mathrm{Mpc}$. The bottom panel of the figure depicts the filament radius as a function of the filament length. We observe that the filament length does not correlate with the filament radius. However, it is important to note that the radius varies slightly around the mean value along the filament path.

\section{Properties of galaxies in filaments}

\subsection{Stellar mass profile}

We constructed a galaxy stellar mass profile for all filaments by using the masses from the MPA-JHU group (Brinchmann et al.

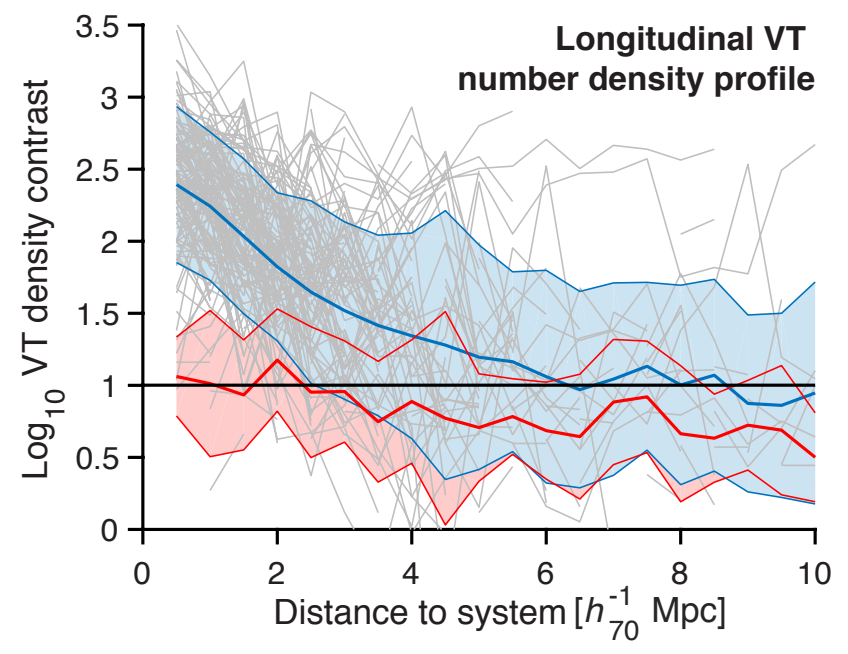

Fig. 13. Longitudinal VT density distribution for galaxies in all bridges of filaments detected by GFiF. Profiles are considered from the system center to the middle of the bridge. The thick blue line depicts the mean longitudinal profile for bridges including galaxies in systems. The thick red line corresponds to the mean longitudinal profile for all filaments excluding galaxies belonging to systems within $1.5 R_{\text {vir }}$. Blue and red shaded areas are the dispersion around the stacked profile.

2004; Kauffmann et al. 2003; Tremonti et al. 2004) described in Sect. 2.2. First, we weighted the mass by the average mass of the volume under analysis to remove the redshift dependence of the stellar mass (Chen et al. 2017). This weighting is equivalent to a normalization of the stellar mass and allows us to carry out a stacking procedure in order to increase the signal of the profiles. This mass profile is extracted as described in Sect. 3.7.

Figure 16 shows the stacked stellar mass profile for all filaments. The variance of the stacked profile is depicted by the error bars. We observe that, statistically, the stellar masses of the filament galaxies are larger than the average mass up to about $2 \mathrm{~h}_{70}^{-1} \mathrm{Mpc}$, while beyond $3 \mathrm{~h}_{70}^{-1} \mathrm{Mpc}$ they tend to be $10 \%$ smaller. This region, farther than $3 \mathrm{~h}_{70}^{-1} \mathrm{Mpc}$, probably represents the dispersed population of the supercluster associated to the more extended sheet component. Therefore, our results indicate that the stellar mass correlates with the distance to the filament skeleton, and is greater (up to 25\%) near the skeleton than far from it. These results are in good agreement with the results presented by Chen et al. (2017) for the MGS sample from DR7 (Abazajian et al. 2009). Our results are also compatible with those presented by Alpaslan et al. (2016) and Kraljic et al. (2018), for the GAMA spectroscopic survey, who find similar trends for the filaments found at redshifts $z<0.09$ and $0.03 \leq z \leq 0.25$, respectively.

\subsection{Morphological type}

In order to decipher whether or not there is some morphological trend in the population of filament galaxies (as may be expected from the morphology-density relation), we also constructed morphology profiles based on morphological classifications by Huertas-Company et al. (2011). These profiles classify the galaxies into four morphological types. For our analysis, we used the probability $p($ Early $)=p(\mathrm{E})+p(\mathrm{~S} 0)$ that classifies galaxies into early type as $p$ (Early) $>0.5$ and late type as $p$ (Early) $<0.5$. We then computed the distribution of both galaxy types as a function of the distance to the filament skeleton. The distributions were normalized so that they can be 

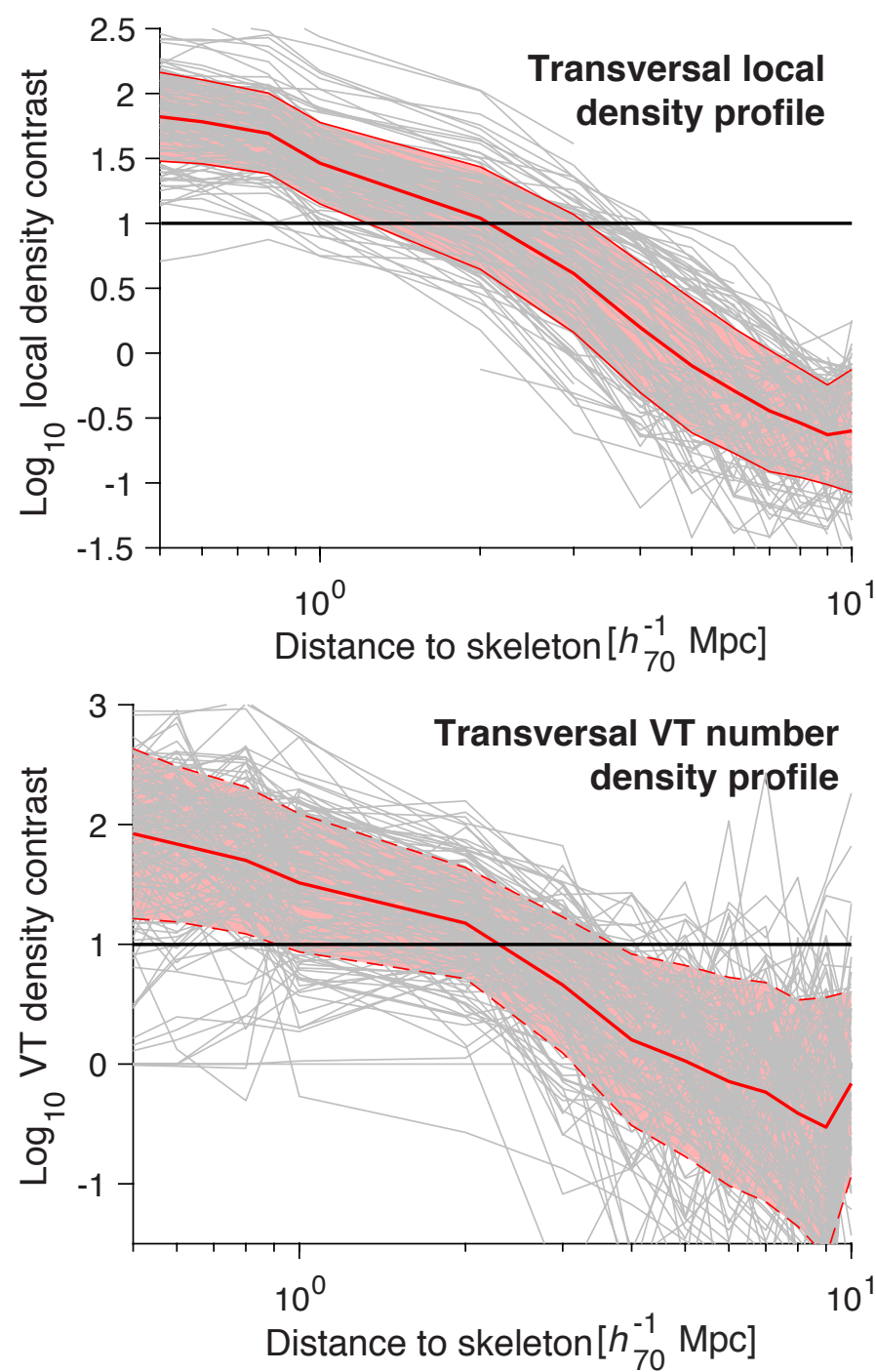

Fig. 14. Stacked number density profiles for the 144 filaments identified by GFiF. Individual profiles are represented by thin gray lines. Top: the red lines corresponds to the mean local density (stacked) profile. Bottom: mean VT density stacked profile. The solid line indicates the mean profile while the shaded area represents the dispersion of the profile. Solid black line depicts the density contrast of $10 \times d$.

compared and stacked for all filaments in our sample in a similar way to a profile extraction, again excluding galaxies in systems. The result is shown in Fig. 17.

Our results show that the fraction of early-type galaxies is higher than that of late types near the filament skeleton up to $\sim 2 \mathrm{~h}_{70}^{-1} \mathrm{Mpc}$. This effect is more discernible when computed as an early-to-late-type ratio (Fig. 17, bottom panel). We observe that at distances shorter than $2 \mathrm{~h}_{70}^{-1} \mathrm{Mpc}$, the fraction of early types reaches almost twice the fraction of late types. At greater distances (i.e., towards the dispersed supercluster population) the fractions tend to be similar (E/S ratio $\sim 1)$. A two-sample Kolmogorov-Smirnov test applied to the distributions of early and late types in Fig. 17 reveals that, for the first bins, they are significantly different ( $p$-value lower than 0.1). Our results are consistent with those presented by Kuutma et al. (2017) for the Huertas-Company et al. (2011) sample - these latter authors also observe that early-type galaxies are more abundant near the filament skeleton.
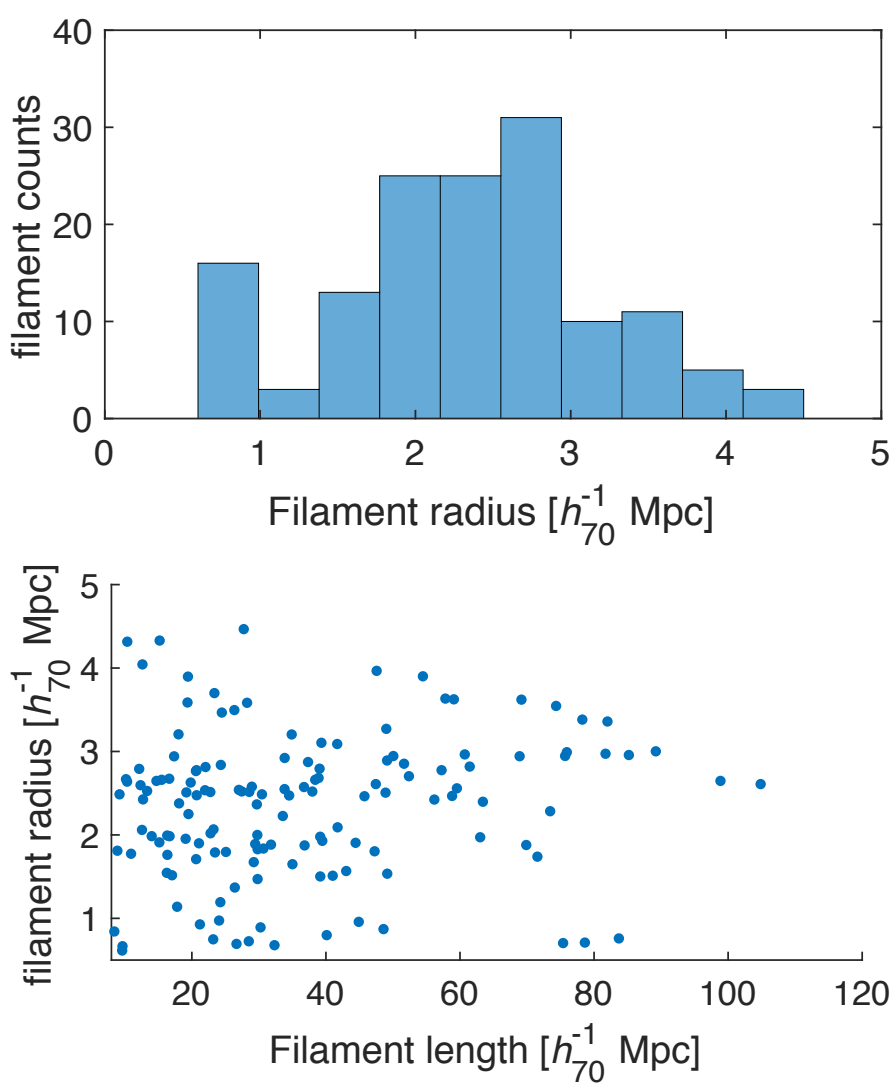

Fig. 15. Top: distribution of radius of filaments in our sample. Bottom: comparison of filament length and radius for the 144 filaments detected by GFiF. The length used corresponds to the longest path between a pair of systems, that is, the skeleton length. See Table A.1.

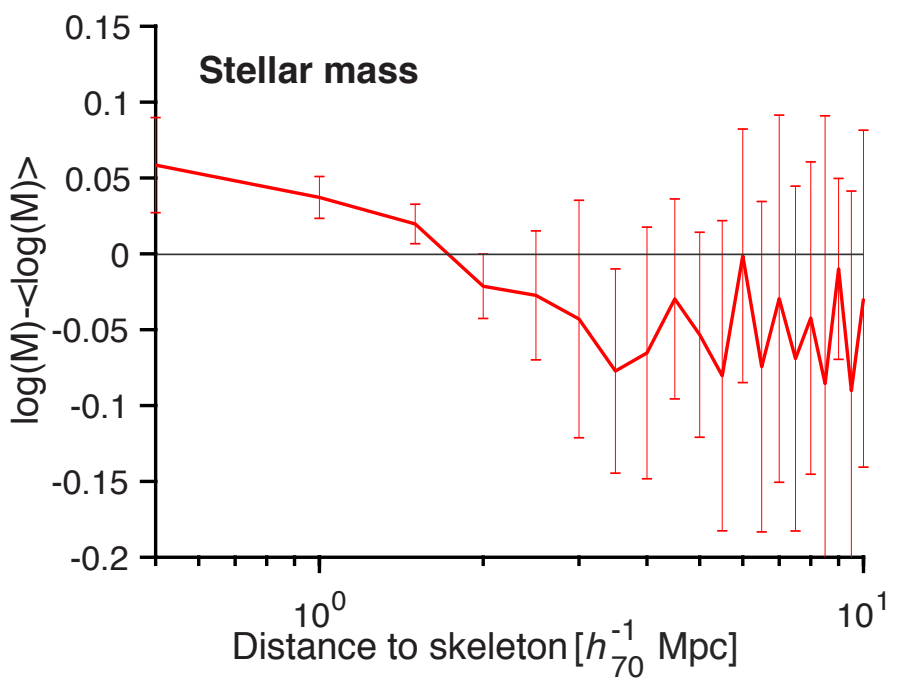

Fig. 16. Stacked transversal stellar mass profile for the 144 filaments detected by GFiF. Errors correspond to the variance of the stacked profiles.

\subsection{Activity type}

We used the activity classification from the MPA-JHU group (Brinchmann et al. 2004; Kauffmann et al. 2003; Tremonti et al. 2004) described in Sect. 2.2 for the analysis with respect to activity type. We computed the distribution of the different galaxy activity populations as a function of the filament 

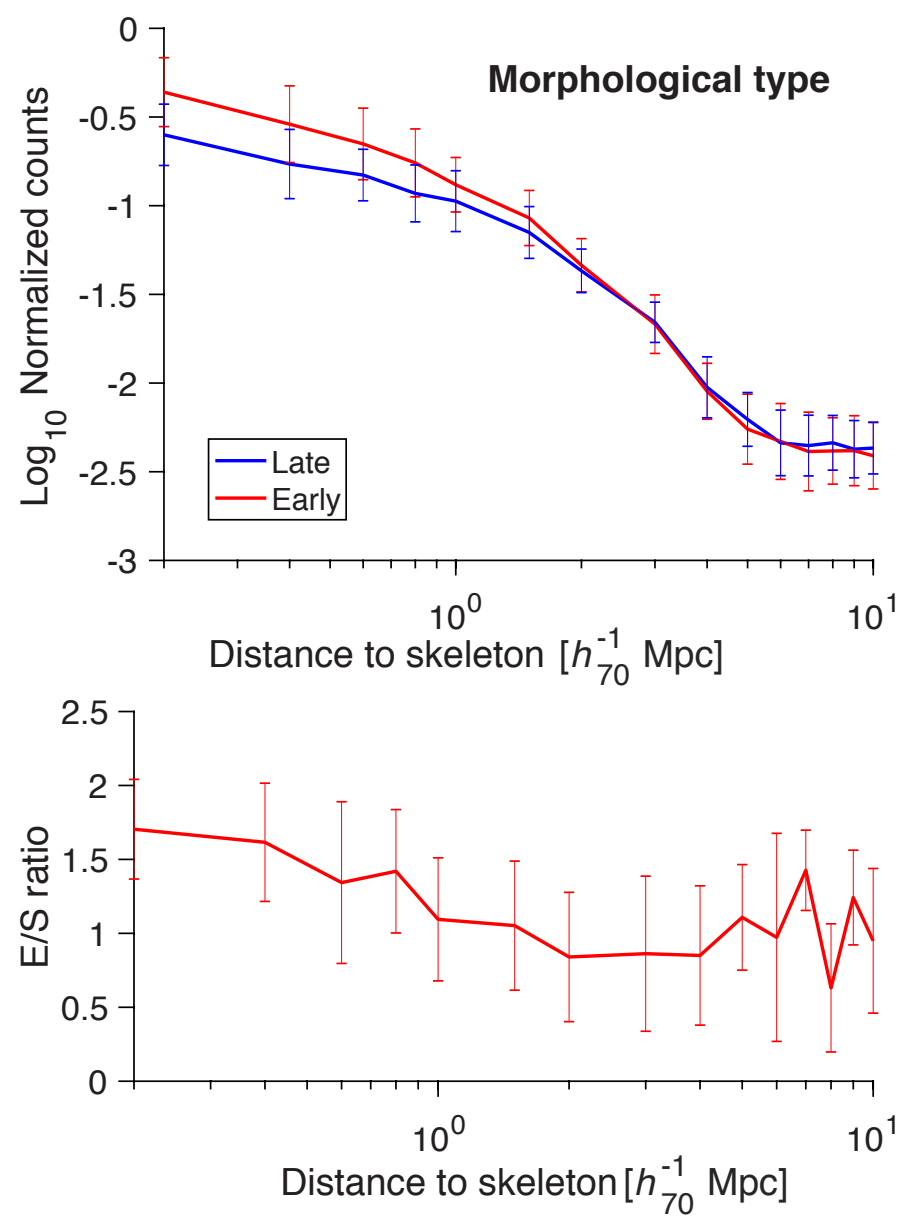

Fig. 17. Top: stacked transversal morphological type profiles for the 144 filaments detected by GFiF. The error bars correspond to the variance of the stacked profiles. Bottom: early-to-late-type ratio as a function of the distance to the filament skeleton.

skeleton distance. All distributions are normalized for all filaments and stacked together.

Figure 18 divides galaxies into four activity groups: active galactic nuclei (AGNs), star-forming galaxies (SFGs), lowionization nuclear emission-line region galaxies (LINERs), and inactive galaxies (unclassified). The error bars are not displayed over the lines for the purpose of clarity - note that they are very large, implying that we have to interpret this figure with caution. Another effect to take into account is that the fractions are averaged over all the filaments (at different redshifts). The most evident tendency we can see in these distributions is a decrease in the activity as long as the galaxies "approach" the filament, although the fractions for the dispersed component are particular noisy. Inside the filaments, the tendency is to have more passive galaxies, implying again smaller fractions of AGNs and SFGs. However, the fraction of LINERs also increases towards the filament skeletons, possibly indicating a post-activity phase for the galaxies. Deeper analyses are necessary to give a clear picture of the effect of the filament environment on the activity of galaxies.

\section{Conclusions}

In this paper we studied the bridges and filaments of galaxies in the environment of superclusters of galaxies. We developed two algorithms, the Galaxy System-Finding algorithm, GSyF, and the Galaxy Filament-Finding algorithm, GFiF, to detect sys-

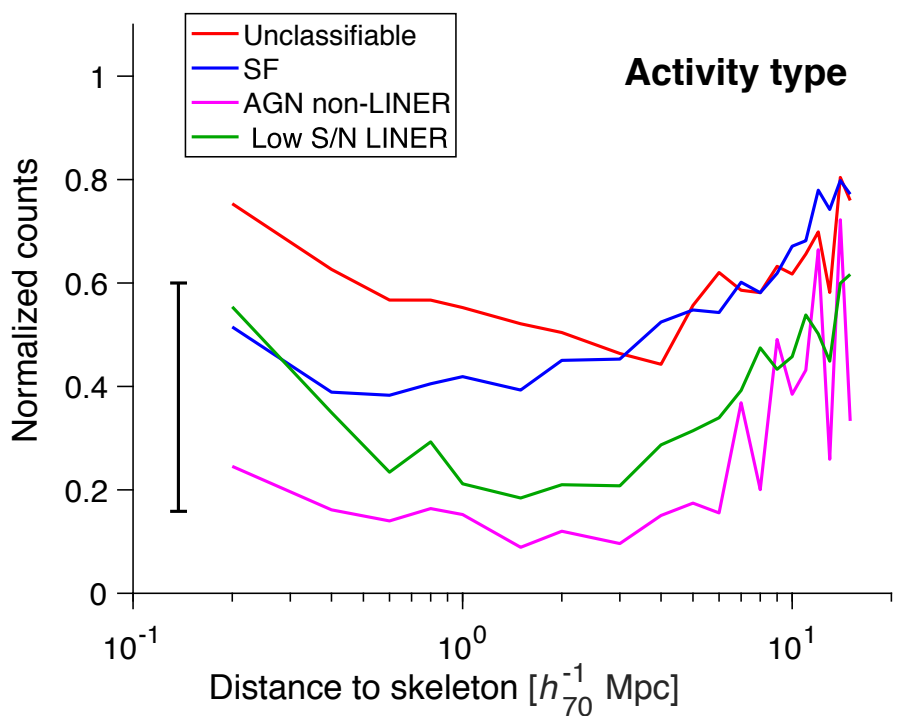

Fig. 18. Stacked transversal activity type profiles for the 144 filaments detected by GFiF. The black bar on the left represents the typical errors on the stacked profiles, not overlaid for clarity.

tems of galaxies (clusters and groups), aiming especially to correct for the FoG effect, and to identify the elongated bridges and filaments mentioned above. These algorithms were applied to a sample of SDSS galaxies with spectroscopic redshifts in rectangular boxes enclosing 46 superclusters of galaxies selected from the MSCC catalog in a redshift range from 0.02 to 0.15 .

GSyF and GFiF employ a set of different classic patternrecognition methods. Both of them are probabilistic in the sense that they define systems and filaments as a function of the relative position and orientation of the Gaussian groups, which are detected with a hierarchical clusterization method. For GSyF, the membership of the Gaussian groups is refined using a virial approximation, allowing us to discern gravitationally bound systems of galaxies from misdetections. For GFiF, these measurements are used to define a general tree from which we extract independent structures based on density criteria. Although the $\mathrm{HC}$ algorithm needs to be optimized for the number of clustering groups, this can be automatized based on the density function characterizing the survey. The structures are represented by a filament skeleton that allows us to measure and quantitatively trace the filament path.

We show (Sect. 7.1) that the systems detected by our methodology are in good agreement with those reported in the literature. Specifically, our comparisons of the systems sample with other cluster and group catalogs (Abell, C4, L14, T11 and MSPM) showed a match rate above $78 \%$ for groups with richness above five galaxies at redshifts $z<0.11$. For systems with richness above ten galaxies the coincidences were slightly higher for the group catalogs (T11 and MSPM) and were slightly lower for the cluster catalogs (Abell and C4). Moreover, the richness, velocity dispersion and virial radius of systems measured by the GSyF algorithm are in good agreement with those reported in other system catalogs. Our GSyF algorithm detected a total of 2705 systems in the rectangular boxes enclosing the volumes of 45 of the superclusters in our sample. Of these, 159 systems with richness above ten galaxies have not yet been reported in the literature $^{3}$.

3 Data on these systems are available at the CDS. 
We also compared, in Sect. 7.2, the results of our filamentfinding algorithm with those of Tempel et al. (2014) for the same regions. We observe that T14 filaments are shorter, more numerous, and describe sparser and finer structures while GFiF detects larger and denser elongated structures that bridge galaxy systems. Our filaments, in some sense, link several T14 threads, forming one larger structure, providing a broader picture of the filament. The comparison with isolated bridges and tendrils (a byproduct of our algorithm) shows a match of $80 \%$ with T14 filaments and comparable filament lengths.

The GFiF algorithm detected a total of 144 filaments and 63 isolated bridges in the rectangular boxes enclosing the volumes of 40 of the superclusters in our sample. The supercluster filaments we detected have lengths from 9 up to $130 \mathrm{~h}_{70}^{-1} \mathrm{Mpc}$ (mean $37 \mathrm{~h}_{70}^{-1} \mathrm{Mpc}$, median $29 \mathrm{~h}_{70}^{-1} \mathrm{Mpc}$ ) while the isolated bridges have lengths of between 5 and $15 \mathrm{~h}_{70}^{-1} \mathrm{Mpc}$. These values are consistent with the median bridge length value from Kraljic et al. (2019), $7.9 \mathrm{~h}_{70}^{-1} \mathrm{Mpc}$, for the HORIZON-AGN simulation.

For most of the cases, the numerical density inside the filaments was found to be between 5 and 15 times the mean density. The radii of the filament skeletons range from 0.6 up to $4.5 \mathrm{~h}_{70}^{-1} \mathrm{Mpc}$, with most being between 2 and $3 \mathrm{~h}_{70}^{-1} \mathrm{Mpc}$. These values are consistent with those found by Cautun et al. (2013) by applying the NEXUS algorithm to an $N$-body simulation.

We also compared the properties of the galaxies that inhabit the filament as a function of the distance from its skeleton. Our conclusions can be summarized as follows: (i) The transversal local and VT number density profiles for pure filaments show that, at distances of up to $5 \mathrm{~h}_{70}^{-1} \mathrm{Mpc}$, the filaments have a positive overdensity with respect to the background density inside the boxes. (ii) At distances of about $3 \mathrm{~h}_{70}^{-1} \mathrm{Mpc}$, the density contrast reaches a value of 3 , a limit that matches the range where typically the environmental effects studied in Sect. 9 seem to apply. (iii) The mean density contrast of the filaments, 10, is reached closer to $2 \mathrm{~h}_{70}^{-1} \mathrm{Mpc}$, a limit that we used as reference for estimating the radius of the filaments.

Our analyses regarding the stellar masses, morphological type, and activity type show that these galaxy properties correlate with the distance from the filament skeleton. We arrive at the following conclusions: (i) Inside $3 \mathrm{~h}_{70}^{-1} \mathrm{Mpc}$ from the filament skeleton the galaxy stellar masses increase up to about $25 \%$. This result leads to two hypotheses: (a) the mass growth of the galaxies is sensitive to the environment, or (b) the dynamical evolution brings massive galaxies into the potential well of the filaments. This result confirms several analyses which suggest that stellar masses are sensitive to the environment (Alpaslan et al. 2015, 2016; Poudel et al. 2016; Chen et al. 2017; Malavasi et al. 2017; Kraljic et al. 2018; Musso et al. 2018). (ii) The early-tolate-type-galaxy ratio has its maximum at the center of the filament and remains above 1:1 up to a distance of $1.5 \mathrm{~h}_{70}^{-1} \mathrm{Mpc}$. This result is in close agreement with a similar study by Kuutma et al. (2017) for the SDSS. (iii) Concerning the activity type, we observe that the fractions of AGNs and SFGs seem to be higher outside the filaments (in the supercluster dispersed component), showing a decrease as the galaxies approach these structures. Inside the filaments, the fractions of inactive galaxies and LINERs increase, indicating a possible post-activity phase. A similar result for the star-forming galaxies was observed by Kraljic et al. (2018) for the GAMA spectroscopic survey.

The GSyF and GFiF algorithms can be used to search for these kinds of structures in different surveys, using spectroscopic or photometric redshifts. We plan to apply them to other galaxy databases, like the ones that are becoming available for the southern celestial hemisphere, and also, to galaxy surveys that reach deeper redshifts. Both algorithms and catalogs can be obtained electronically upon request.

Acknowledgements. I.S.-B. thanks CONACyT and DAIP for funding this research. This project was partially financed by DAIP funding CIIC 205/2019. The authors are grateful to the anonymous referee for the important comments that helped to improve the paper. All the pattern recognition computing, statistics and graphics have been made using MATLAB(C). Part of this work was carried out with the computational facility TITAN at the Institut de Recherche en Astrophysique et Planétologie, Toulouse, France. This work has made use of NASA's Astrophysics Data System Bibliographic Services. Funding for SDSSIII has been provided by the Alfred P. Sloan Foundation, the Participating Institutions, the National Science Foundation, and the US Department of Energy Office of Science. The SDSS-III web site is http://www.sdss3.org/. SDSS-III is managed by the Astrophysical Research Consortium for the Participating Institutions of the SDSS-III Collaboration including the University of Arizona, the Brazilian Participation Group, Brookhaven National Laboratory, Carnegie Mellon University, University of Florida, the French Participation Group, the German Participation Group, Harvard University, the Instituto de Astrofisica de Canarias, the Michigan State/Notre Dame/JINA Participation Group, Johns Hopkins University, Lawrence Berkeley National Laboratory, Max Planck Institute for Astrophysics, Max Planck Institute for Extraterrestrial Physics, New Mexico State University, New York University, Ohio State University, Pennsylvania State University, University of Portsmouth, Princeton University, the Spanish Participation Group, University of Tokyo, University of Utah, Vanderbilt University, University of Virginia, University of Washington, and Yale University.

\section{References}

Abazajian, K. N., Adelman-McCarthy, J. K., Agüeros, M. A., et al. 2009, ApJS, 182,543

Abell, G. O. 1961, AJ, 66, 607

Abell, G. O., Corwin, H. G., Jr, \& Olowin, R. P. 1989, ApJS, 70,

Aihara, H., Allende Prieto, C., An, D., et al. 2011, ApJS, 193, 29

Alam, S., Albareti, F. D., Allende Prieto, C., et al. 2015, ApJS, 219, 12

Albareti, F. D., Allende Prieto, C., Almeida, A., et al. 2017, ApJS, 233, 25

Alpaslan, M., Robotham, A. S. G., Obreschkow, D., et al. 2014, MNRAS, 440, L106

Alpaslan, M., Driver, S., Robotham, A. S. G., et al. 2015, MNRAS, 451, 3249

Alpaslan, M., Grootes, M., Marcum, P. M., et al. 2016, MNRAS, 457, 2287

Alvarez, G. E., Randall, S. W., Bourdin, H., Jones, C., \& Holley-Bockelmann, K. 2018, ApJ, 858, 44

Aragón-Calvo, M. A., Jones, B. J. T., van de Weygaert, R., \& van der Hulst, J. M. 2007, A\&A, 474, 315

Aragón-Calvo, M. A., Platen, E., van de Weygaert, R., \& Szalay, A. S. 2010, ApJ, 723, 364

Beers, T. C., Flynn, K., \& Gebhardt, K. 1990, AJ, 100, 32

Biviano, A., Murante, G., Borgani, S., et al. 2006, A\&A, 456, 23

Bolton, A. S., Schlegel, D. J., Aubourg, É., et al. 2012, AJ, 144, 144

Bond, J. R., \& Szalay, A. S. 1983, ApJ, 274, 443

Bond, J. R., Kofman, L., \& Pogosyan, D. 1996, Nature, 380, 603

Bond, N. A., Strauss, M. A., \& Cen, R. 2010, MNRAS, 409, 156

Brinchmann, J., Charlot, S., White, S. D. M., et al. 2004, MNRAS, 351, 1151

Cautun, M., van de Weygaert, R., \& Jones, B. J. T. 2013, MNRAS, 429, 1286

Cen, R., \& Ostriker, J. P. 1999, ApJ, 514, 1

Chen, Y.-C., Ho, S., Freeman, P. E., Genovese, C. R., \& Wasserman, L. 2015 , MNRAS, 454, 1140

Chen, Y.-C., Ho, S., Brinkmann, J., et al. 2016, MNRAS, 461, 3896

Chen, Y.-C., Ho, S., Mandelbaum, R., et al. 2017, MNRAS, 466, 1880

Chow-Martinez, M., Andernach, H., Caretta, C. A., \& Trejo-Alonso, J. J. 2014, MNRAS, 445, 4073

Colless, M., Dalton, G., Maddox, S., et al. 2001, MNRAS, 328, 1039

Costa-Duarte, M. V., Sodré, L., Jr, \& Durret, F. 2011, MNRAS, 411, 1716

Cybulski, R., Yun, M. S., Fazio, G. G., \& Gutermuth, R. A. 2014, MNRAS, 439, 3564

Darvish, B., Mobasher, B., Sobral, D., Scoville, N., \& Aragon-Calvo, M. 2015, ApJ, 805, 121

Davis, M., Huchra, J., Latham, D. W., \& Tonry, J. 1982, ApJ, 253, 423

Dijkstra, E. W. 1959, Numer. Math., 1, 269

Doroshkevich, A. G., \& Khlopov, M. I. 1984, MNRAS, 211, 277

Dupuy, A., Courtois, H. M., Dupont, F., et al. 2019, MNRAS, 489, L1

Eckert, D., Ettori, S., Pointecouteau, E., et al. 2017, Astron. Nachr., 338, 293

Einasto, M., Einasto, J., Tago, E., Müller, V., \& Andernach, H. 2001, AJ, 122, 2222

Einasto, M., Saar, E., Martínez, V. J., et al. 2008, ApJ, 685, 83 
Einasto, M., Lietzen, H., Tempel, E., et al. 2014, A\&A, 562, A87

Einasto, J., Suhhonenko, I., Liivamägi, L. J., \& Einasto, M. 2019, A\&A, 623, A97

Eisenstein, D. J., Zehavi, I., Hogg, D. W., et al. 2005, ApJ, 633, 560

Gallazzi, A., Bell, E. F., Wolf, C., et al. 2009, ApJ, 690, 1883

Gavazzi, G., Fumagalli, M., Cucciati, O., \& Boselli, A. 2010, A\&A, 517, A73

González, R. E., \& Padilla, N. D. 2010, MNRAS, 407, 1449

Graham, R. L., \& Hell, P. 1985, Ann. History Comput., 7, 43

Guennou, L., Adami, C., Durret, F., et al. 2014, A\&A, 561, A112

Guglielmo, V., Poggianti, B. M., Vulcani, B., et al. 2018, A\&A, 620, A7

Huchra, J. P., Macri, L. M., Masters, K. L., et al. 2012, ApJS, 199, 26

Huertas-Company, M., Aguerri, J. A. L., Bernardi, M., Mei, S., \& Sánchez Almeida, J. 2011, A\&A, 525, A157

Kauffmann, G., Heckman, T. M., White, S. D. M., et al. 2003, MNRAS, 341, 33

Klypin, A. A., Trujillo-Gomez, S., \& Primack, J. 2011, ApJ, 740, 102

Kopylova, F. G., \& Kopylov, A. I. 2006, Astron. Lett., 32, 84

Kraljic, K., Arnouts, S., Pichon, C., et al. 2018, MNRAS, 474, 547

Kraljic, K., Pichon, C., Dubois, Y., et al. 2019, MNRAS, 483, 3227

Krause, M. O., Ribeiro, A. L. B., \& Lopes, P. A. A. 2013, A\&A, 551, A143

Kuutma, T., Tamm, A., \& Tempel, E. 2017, A\&A, 600, L6

Lauer, T. R., Postman, M., Strauss, M. A., Graves, G. J., \& Chisari, N. E. 2014, ApJ, 797, 82

Libeskind, N. I., van de Weygaert, R., Cautun, M., et al. 2018, MNRAS, 473, 1195

Liivamägi, L. J., Tempel, E., \& Saar, E. 2012, A\&A, 539, A80

Lintott, C. J., Schawinski, K., Slosar, A., et al. 2008, MNRAS, 389, 1179

Lintott, C., Schawinski, K., Bamford, S., et al. 2011, MNRAS, 410, 166

Luparello, H., Lares, M., Lambas, D. G., \& Padilla, N. 2011, MNRAS, 415, 964

Malavasi, N., Arnouts, S., Vibert, D., et al. 2017, MNRAS, 465, 3817

Miller, C. J., Nichol, R. C., Reichart, D., et al. 2005, ApJ, 130, 968

Murtagh, F., \& Contreras, P. 2011, Wiley Interdisciplinary Rev.: Data Mining Knowl. Discovery, 2, 86

Murtagh, F., \& Legendre, P. 2014, J. Classification, 31, 274

Musso, M., Cadiou, C., Pichon, C., et al. 2018, MNRAS, 476, 4877

Peebles, P. J. E. 1980, The Large-scale Structure of the Universe (Princeton University Press)

Planck Collaboration VIII. 2013, A\&A, 550, A134

Platen, E., van de Weygaert, R., Jones, B. J. T., Vegter, G., \& Calvo, M. A. A 2011, MNRAS, 416, 2494
Poudel, A., Heinämäki, P., Nurmi, P., et al. 2016, A\&A, 590, A29

Rines, K., Geller, M. J., Kurtz, M. J., \& Diaferio, A. 2003, AJ, 126, 2152

Rines, K., Diaferio, A., \& Natarajan, P. 2007, ApJ, 657, 183

Santanu, R. S. 2014, Graph Theory with Algorithms and Its Applications: In Applied Science and Technology (Springer Publishing Company, Incorporated)

Santiago-Bautista, I., Caretta, C. A., Bravo-Alfaro, H., Pointecouteau, E., \& Madrigal, F. 2019, ArXiv e-prints [arXiv:2001.03209]

Scoville, N., Arnouts, S., Aussel, H., et al. 2013, ApJS, 206, 3

Serna, A., \& Gerbal, D. 1996, A\&A, 309, 65

Shandarin, S. F., \& Zel'dovich, Y. B. 1989, Rev. Mod. Phys., 61, 185

Smargon, A., Mandelbaum, R., Bahcall, N., \& Niederste-Ostholt, M. 2012, MNRAS, 423, 856

Smith, A. G., Hopkins, A. M., Hunstead, R. W., \& Pimbblet, K. A. 2012, MNRAS, 422, 25

Sousbie, T. 2011, MNRAS, 414, 350

Sousbie, T., Pichon, C., Courtois, H., Colombi, S., \& Novikov, D. 2008, ApJ, 672, L1

Springel, V., White, S. D. M., Jenkins, A., et al. 2005, Nature, 435, 629

Strauss, M. A., Weinberg, D. H., Lupton, R. H., et al. 2002, AJ, 124, 1810

Tanaka, M., Hoshi, T., Kodama, T., \& Kashikawa, N. 2007, MNRAS, 379, 1546

Tanimura, H., Aghanim, N., Douspis, M., Beelen, A., \& Bonjean, V. 2019, A\&A, 625, A67

Tempel, E., Tago, E., \& Liivamägi, L. J. 2012, A\&A, 540, A106

Tempel, E., Stoica, R. S., Martínez, V. J., et al. 2014, MNRAS, 438, 3465

Theodoridis, S., \& Koutroumbas, K. 2009, in Pattern Recognition, 4th edn., eds. T. Sergios, \& K. Konstantinos (Boston: Academic Press), 595

Theodoridis, S., Pikrakis, A., Koutroumbas, K., \& Cavouras, D. 2010, Introduction to Pattern Recognition: A Matlab Approach (Boston: Academic Press)

Tremonti, C. A., Heckman, T. M., Kauffmann, G., et al. 2004, ApJ, 613, 898

Tully, R. B., Courtois, H., Hoffman, Y., \& Pomarède, D. 2014, Nature, 513, 71

Ueda, H., \& Itoh, M. 1997, PASJ, 49, 131

Ursino, E., Galeazzi, M., Gupta, A., et al. 2015, ApJ, 806, 211

Vogelsberger, M., Genel, S., Springel, V., et al. 2014, Nature, 509, 177

Voronoi, G. 1908, J. Reine Angew. Math., 134, 198

Wang, P., Luo, Y., Kang, X., et al. 2018, ApJ, 859, 115 


\section{Appendix A: Additional table}

Table A.1. Main properties of the filaments extracted through GFiF.

\begin{tabular}{|c|c|c|c|c|c|c|c|c|c|c|}
\hline \multirow{2}{*}{$\begin{array}{l}\text { Fil. } \\
\text { ID } \\
(1)\end{array}$} & \multirow{2}{*}{$\begin{array}{r}N_{\text {sfil }} \\
\text { systems } \\
(2)\end{array}$} & \multirow{2}{*}{$\begin{array}{r}N_{\text {gfil }} \\
\text { gals. } \\
(3) \\
\end{array}$} & \multicolumn{3}{|c|}{ Redshift } & \multirow{2}{*}{$\begin{aligned} & \begin{array}{l}d_{\text {fil }} \\
{\left[\mathrm{h}_{70}^{3} \mathrm{Mpc}^{-3}\right]} \\
(7)\end{array} \\
&\end{aligned}$} & \multirow{2}{*}{$\begin{array}{c}R_{\mathrm{fil}} \\
{\left[\mathrm{h}_{70}^{-1} \mathrm{Mpc}\right]} \\
\quad(8)\end{array}$} & \multicolumn{2}{|c|}{$N_{\text {nod }}$} & \multirow{2}{*}{$\begin{array}{r}\ell_{\text {fil }} \\
{\left[\mathrm{h}_{70}^{-1} \mathrm{Mpc}\right]} \\
(11)\end{array}$} \\
\hline & & & $\begin{array}{c}\text { [mean, } \\
(4)\end{array}$ & $\begin{array}{c}\min , \\
(5)\end{array}$ & $\begin{array}{c}\max ] \\
(6)\end{array}$ & & & $\begin{array}{r}\text { Filament } \\
(9) \\
\end{array}$ & $\begin{array}{r}\text { Skeleton } \\
(10) \\
\end{array}$ & \\
\hline MSCC-55-F1 & 4 & 119 & 0.0619 & 0.0529 & 0.0706 & 0.1570 & 4.31 & 3 & 2 & 10.5 \\
\hline MSCC-55-F2 & 3 & 2 & 0.0585 & 0.0527 & 0.0680 & 0.1911 & 0.60 & 2 & 2 & 9.7 \\
\hline MSCC-72-F1 & 5 & 257 & 0.0790 & 0.0722 & 0.0868 & 0.5636 & 3.57 & 6 & 6 & 28.4 \\
\hline MSCC-72-F2 & 4 & 217 & 0.0801 & 0.0718 & 0.0866 & 0.3688 & 3.46 & 5 & 5 & 24.6 \\
\hline MSCC-72-F3 & 5 & 215 & 0.0777 & 0.0717 & 0.0839 & 0.3043 & 3.89 & 6 & 5 & 19.5 \\
\hline MSCC-72-F4 & 3 & 140 & 0.0850 & 0.0787 & 0.0917 & 0.2012 & 3.49 & 4 & 4 & 26.5 \\
\hline MSCC-175-F1 & 6 & 156 & 0.0912 & 0.0841 & 0.0996 & 0.1541 & 3.08 & 8 & 6 & 41.8 \\
\hline MSCC-175-F2 & 3 & 159 & 0.0978 & 0.0885 & 0.1032 & 0.2260 & 3.19 & 3 & 3 & 35.0 \\
\hline MSCC-175-F3 & 3 & 87 & 0.0937 & 0.0883 & 0.1001 & 0.1714 & 1.79 & 5 & 4 & 47.4 \\
\hline MSCC-175-F4 & 3 & 105 & 0.0923 & 0.0867 & 0.0995 & 0.2184 & 2.86 & 3 & 3 & 37.5 \\
\hline MSCC-184-F1 & 5 & 157 & 0.1071 & 0.0994 & 0.1171 & 0.0488 & 2.88 & 8 & 5 & 49.3 \\
\hline MSCC-184-F2 & 3 & 61 & 0.0980 & 0.0911 & 0.1029 & 0.0838 & 3.09 & 3 & 3 & 39.5 \\
\hline MSCC-211-F1 & 3 & 233 & 0.1205 & 0.1100 & 0.1297 & 0.0465 & 4.46 & 13 & 8 & 27.9 \\
\hline MSCC-219-F1 & 10 & 275 & 0.1125 & 0.1063 & 0.1207 & 0.1169 & 2.96 & 17 & 6 & 81.8 \\
\hline MSCC-219-F2 & 3 & 4 & 0.1235 & 0.1183 & 0.1287 & 0.0347 & 0.79 & 3 & 3 & 40.2 \\
\hline MSCC-222-F1 & 3 & 89 & 0.1422 & 0.1311 & 0.1522 & 0.0418 & 1.87 & 14 & 8 & 70.0 \\
\hline MSCC-222-F2 & 5 & 8 & 0.1349 & 0.1238 & 0.1460 & 0.0461 & 0.70 & 11 & 8 & 78.8 \\
\hline MSCC-223-F1 & 3 & 11 & 0.1367 & 0.1286 & 0.1472 & 0.0245 & 0.86 & 12 & 8 & 48.7 \\
\hline MSCC-229-F1 & 3 & 32 & 0.1445 & 0.1361 & 0.1514 & 0.0389 & 1.96 & 9 & 6 & 63.1 \\
\hline MSCC-236-F1 & 5 & 231 & 0.0324 & 0.0228 & 0.0415 & 0.3382 & 1.49 & 16 & 9 & 39.3 \\
\hline MSCC-236-F2 & 4 & 324 & 0.0411 & 0.0361 & 0.0461 & 0.2409 & 2.65 & 7 & 6 & 38.5 \\
\hline MSCC-236-F3 & 5 & 286 & 0.0331 & 0.0274 & 0.0399 & 0.4889 & 2.62 & 8 & 5 & 20.0 \\
\hline MSCC-236-F4 & 3 & 150 & 0.0296 & 0.0255 & 0.0364 & 0.3971 & 2.24 & 5 & 4 & 19.6 \\
\hline MSCC-236-F5 & 3 & 188 & 0.0354 & 0.0286 & 0.0422 & 0.4448 & 2.47 & 5 & 5 & 20.8 \\
\hline MSCC-236-F6 & 3 & 318 & 0.0330 & 0.0276 & 0.0373 & 2.3032 & 2.66 & 2 & 2 & 16.8 \\
\hline MSCC-236-F7 & 3 & 103 & 0.0333 & 0.0297 & 0.0391 & 0.4359 & 1.53 & 4 & 3 & 16.4 \\
\hline MSCC-238-F1 & 5 & 174 & 0.1190 & 0.1075 & 0.1309 & 0.0558 & 2.95 & 11 & 7 & 85.3 \\
\hline MSCC-238-F2 & 6 & 201 & 0.0915 & 0.0802 & 0.1010 & 0.0928 & 2.98 & 14 & 10 & 76.1 \\
\hline MSCC-238-F3 & 3 & 155 & 0.1054 & 0.0979 & 0.1115 & 0.0782 & 2.64 & 11 & 8 & 99.0 \\
\hline MSCC-238-F4 & 3 & 3 & 0.0957 & 0.0896 & 0.1024 & 0.1467 & 0.71 & 3 & 3 & 28.6 \\
\hline MSCC-238-F5 & 3 & 4 & 0.1016 & 0.0934 & 0.1099 & 0.0285 & 0.67 & 3 & 2 & 32.5 \\
\hline MSCC-238-F6 & 3 & 7 & 0.1096 & 0.1040 & 0.1156 & 0.0398 & 0.88 & 4 & 3 & 30.4 \\
\hline MSCC-248-F1 & 3 & 159 & 0.1256 & 0.1161 & 0.1355 & 0.0895 & 3.35 & 10 & 7 & 82.1 \\
\hline MSCC-266-F1 & 5 & 132 & 0.1282 & 0.1188 & 0.1344 & 0.0366 & 2.94 & 9 & 8 & 75.8 \\
\hline MSCC-272-F1 & 6 & 374 & 0.0752 & 0.0694 & 0.0808 & 0.8405 & 2.93 & 6 & 4 & 17.5 \\
\hline MSCC-272-F2 & 3 & 79 & 0.0757 & 0.0714 & 0.0814 & 0.1245 & 2.66 & 4 & 3 & 10.3 \\
\hline MSCC-277-F1 & 10 & 384 & 0.1124 & 0.1031 & 0.1208 & 0.1268 & 2.99 & 21 & 11 & 89.3 \\
\hline MSCC-277-F2 & 5 & 227 & 0.1053 & 0.0956 & 0.1135 & 0.0805 & 2.93 & 16 & 10 & 69.0 \\
\hline MSCC-278-F1 & 7 & 956 & 0.0328 & 0.0251 & 0.0398 & 0.9871 & 2.41 & 36 & 14 & 56.3 \\
\hline MSCC-278-F2 & 3 & 292 & 0.0322 & 0.0273 & 0.0361 & 0.4963 & 1.70 & 24 & 13 & 20.8 \\
\hline MSCC-278-F3 & 4 & 145 & 0.0319 & 0.0266 & 0.0386 & 0.3810 & 1.18 & 14 & 7 & 24.4 \\
\hline MSCC-278-F4 & 8 & 629 & 0.0254 & 0.0221 & 0.0298 & 1.0335 & 1.86 & 27 & 10 & 36.9 \\
\hline MSCC-278-F5 & 3 & 122 & 0.0348 & 0.0297 & 0.0387 & 0.4401 & 1.76 & 7 & 6 & 11.1 \\
\hline MSCC-283-F1 & 4 & 165 & 0.1339 & 0.1245 & 0.1470 & 0.0701 & 3.96 & 7 & 4 & 47.7 \\
\hline MSCC-283-F2 & 5 & 94 & 0.1364 & 0.1284 & 0.1465 & 0.0655 & 3.26 & 6 & 5 & 49.1 \\
\hline MSCC-283-F3 & 3 & 4 & 0.1357 & 0.1296 & 0.1496 & 0.0397 & 0.96 & 2 & 2 & 24.2 \\
\hline MSCC-295-F1 & 7 & 1020 & 0.0230 & 0.0160 & 0.0284 & 2.1243 & 1.89 & 37 & 28 & 44.6 \\
\hline MSCC-295-F2 & 7 & 1289 & 0.0228 & 0.0158 & 0.0299 & 2.2694 & 2.37 & 34 & 12 & 18.2 \\
\hline
\end{tabular}

Notes. (1) ID of the filament; (2) number of systems detected by GSyF linked by the filament; (3) number of galaxies attributed to the filament; (4)-(6) mean, min. and max. redshift of the filament; (7) mean galaxy number density inside the filament; (8) mean transversal radius of the filament measured at $10 \times d$; (9) and (10) number of nodes that constitute the filament and its central skeleton; (11) length of the filament skeleton. 
Table A.1. continued.

\begin{tabular}{|c|c|c|c|c|c|c|c|c|c|c|}
\hline \multirow{2}{*}{$\begin{array}{l}\text { Fil. } \\
\text { ID } \\
(1)\end{array}$} & \multirow{2}{*}{$\begin{array}{r}N_{\text {sfil }} \\
\text { systems } \\
(2)\end{array}$} & \multirow{2}{*}{$\begin{array}{r}N_{\text {gfil }} \\
\text { gals. } \\
(3) \\
\end{array}$} & \multicolumn{3}{|c|}{ Redshift } & \multirow{2}{*}{$\begin{array}{l}d_{\text {fil }} \\
{\left[\mathrm{h}_{70}^{3} \mathrm{Mpc}^{-3}\right]} \\
(7)\end{array}$} & \multirow{2}{*}{$\begin{array}{c}R_{\mathrm{fil}} \\
{\left[\mathrm{h}_{70}^{-1} \mathrm{Mpc}\right]} \\
\quad(8)\end{array}$} & \multicolumn{2}{|c|}{$N_{\text {nod }}$} & \multirow{2}{*}{$\begin{array}{r}\ell_{\text {fil }} \\
{\left[\mathrm{h}_{70}^{-1} \mathrm{Mpc}\right]} \\
(11)\end{array}$} \\
\hline & & & $\begin{array}{l}\text { [mean, } \\
\text { (4) }\end{array}$ & min, & $\begin{array}{c}\max ] \\
(6)\end{array}$ & & & $\begin{array}{r}\text { Filament } \\
(9)\end{array}$ & $\begin{array}{r}\text { Skeleton } \\
(10)\end{array}$ & \\
\hline MSCC-295-F3 & 4 & 497 & 0.0232 & 0.0189 & 0.0283 & 0.5824 & 1.36 & 44 & 17 & 26.5 \\
\hline MSCC-295-F4 & 4 & 186 & 0.0218 & 0.0188 & 0.0255 & 0.6254 & 1.83 & 10 & 9 & 30.8 \\
\hline MSCC-310-F1 & 10 & 499 & 0.0609 & 0.0518 & 0.0689 & 0.4180 & 2.81 & 18 & 11 & 61.6 \\
\hline MSCC-310-F2 & 8 & 523 & 0.0502 & 0.0443 & 0.0588 & 0.5210 & 2.84 & 22 & 11 & 51.8 \\
\hline MSCC-310-F3 & 7 & 407 & 0.0481 & 0.0427 & 0.0528 & 0.3486 & 2.50 & 19 & 10 & 49.0 \\
\hline MSCC-310-F4 & 8 & 313 & 0.0656 & 0.0585 & 0.0710 & 0.4263 & 2.46 & 14 & 10 & 59.0 \\
\hline MSCC-310-F5 & 7 & 325 & 0.0700 & 0.0642 & 0.0774 & 0.5303 & 2.60 & 13 & 7 & 47.6 \\
\hline MSCC-310-F6 & 6 & 243 & 0.0725 & 0.0651 & 0.0791 & 0.2986 & 1.97 & 12 & 9 & 39.3 \\
\hline MSCC-310-F7 & 4 & 219 & 0.0551 & 0.0479 & 0.0619 & 0.4062 & 2.76 & 7 & 5 & 20.7 \\
\hline MSCC-310-F8 & 4 & 164 & 0.0546 & 0.0485 & 0.0617 & 0.2118 & 2.22 & 9 & 7 & 33.7 \\
\hline MSCC-310-F9 & 4 & 124 & 0.0464 & 0.0437 & 0.0528 & 0.1464 & 1.13 & 9 & 6 & 17.9 \\
\hline MSCC-311-F1 & 13 & 607 & 0.0813 & 0.0753 & 0.0892 & 0.2812 & 3.54 & 14 & 9 & 74.5 \\
\hline MSCC-311-F2 & 5 & 254 & 0.0847 & 0.0769 & 0.0937 & 0.3235 & 3.69 & 4 & 3 & 23.5 \\
\hline MSCC-311-F3 & 3 & 138 & 0.0888 & 0.0821 & 0.0937 & 0.1900 & 4.32 & 2 & 2 & 15.3 \\
\hline MSCC-311-F4 & 3 & 119 & 0.0826 & 0.0746 & 0.0901 & 0.2447 & 3.58 & 2 & 2 & 19.5 \\
\hline MSCC-314-F1 & 4 & 46 & 0.0819 & 0.0769 & 0.0893 & 0.1201 & 1.88 & 4 & 4 & 29.6 \\
\hline MSCC-314-F2 & 3 & 66 & 0.0777 & 0.0715 & 0.0844 & 0.1610 & 2.78 & 2 & 2 & 12.3 \\
\hline MSCC-317-F1 & 4 & 55 & 0.1331 & 0.1224 & 0.1417 & 0.0282 & 1.73 & 15 & 10 & 71.7 \\
\hline MSCC-317-F2 & 4 & 8 & 0.1187 & 0.1102 & 0.1264 & 0.0528 & 0.69 & 10 & 7 & 75.5 \\
\hline MSCC-323-F1 & 7 & 223 & 0.1380 & 0.1262 & 0.1540 & 0.0436 & 3.73 & 14 & 11 & 129.3 \\
\hline MSCC-323-F2 & 3 & 16 & 0.1383 & 0.1309 & 0.1535 & 0.0261 & 1.66 & 5 & 4 & 29.4 \\
\hline MSCC-333-F1 & 3 & 82 & 0.0760 & 0.0723 & 0.0833 & 0.1205 & 2.36 & 5 & 4 & 29.8 \\
\hline MSCC-333-F2 & 4 & 117 & 0.0803 & 0.0752 & 0.0880 & 0.1519 & 2.83 & 5 & 4 & 24.5 \\
\hline MSCC-333-F3 & 4 & 83 & 0.0794 & 0.0729 & 0.0835 & 0.2052 & 2.46 & 5 & 5 & 34.6 \\
\hline MSCC-335-F1 & 10 & 345 & 0.0779 & 0.0705 & 0.0843 & 0.1260 & 2.60 & 21 & 11 & 105.0 \\
\hline MSCC-335-F2 & 4 & 22 & 0.0665 & 0.0613 & 0.0731 & 0.0650 & 0.75 & 9 & 8 & 83.8 \\
\hline MSCC-335-F3 & 4 & 111 & 0.0762 & 0.0712 & 0.0814 & 0.1113 & 2.45 & 6 & 4 & 45.9 \\
\hline MSCC-343-F1 & 4 & 115 & 0.0822 & 0.0786 & 0.0874 & 0.1394 & 2.51 & 7 & 6 & 28.7 \\
\hline MSCC-343-F2 & 3 & 95 & 0.0798 & 0.0733 & 0.0876 & 0.2638 & 2.50 & 3 & 3 & 19.3 \\
\hline MSCC-343-F3 & 3 & 125 & 0.0824 & 0.0738 & 0.0876 & 0.1991 & 2.65 & 6 & 5 & 15.6 \\
\hline MSCC-360-F1 & 4 & 182 & 0.1050 & 0.0967 & 0.1159 & 0.1367 & 2.95 & 9 & 6 & 60.8 \\
\hline MSCC-360-F2 & 3 & 31 & 0.1074 & 0.1004 & 0.1152 & 0.0870 & 1.52 & 6 & 4 & 49.3 \\
\hline MSCC-360-F3 & 3 & 5 & 0.1033 & 0.0973 & 0.1093 & 0.0416 & 1.46 & 3 & 2 & 29.9 \\
\hline MSCC-386-F1 & 7 & 346 & 0.0734 & 0.0657 & 0.0805 & 0.4163 & 2.91 & 18 & 10 & 34.0 \\
\hline MSCC-386-F2 & 4 & 115 & 0.0708 & 0.0636 & 0.0765 & 0.1969 & 1.92 & 10 & 8 & 39.6 \\
\hline MSCC-386-F3 & 5 & 129 & 0.0627 & 0.0591 & 0.0705 & 0.1760 & 1.56 & 13 & 10 & 43.2 \\
\hline MSCC-386-F4 & 4 & 46 & 0.0617 & 0.0594 & 0.0664 & 0.1703 & 0.92 & 7 & 6 & 21.3 \\
\hline MSCC-407-F1 & 5 & 101 & 0.1388 & 0.1254 & 0.1468 & 0.0177 & 3.61 & 10 & 6 & 59.2 \\
\hline MSCC-414-F1 & 4 & 420 & 0.0626 & 0.0537 & 0.0691 & 0.6269 & 2.51 & 19 & 9 & 38.1 \\
\hline MSCC-414-F2 & 6 & 243 & 0.0628 & 0.0548 & 0.0667 & 0.3793 & 2.54 & 12 & 7 & 34.0 \\
\hline MSCC-414-F3 & 6 & 240 & 0.0750 & 0.0688 & 0.0809 & 0.5198 & 2.53 & 12 & 7 & 27.1 \\
\hline MSCC-414-F4 & 5 & 196 & 0.0641 & 0.0585 & 0.0675 & 0.3705 & 2.52 & 10 & 5 & 13.5 \\
\hline MSCC-414-F5 & 4 & 243 & 0.0748 & 0.0684 & 0.0810 & 0.4251 & 2.53 & 10 & 8 & 22.1 \\
\hline MSCC-414-F6 & 3 & 126 & 0.0657 & 0.0613 & 0.0701 & 0.3368 & 2.01 & 7 & 6 & 22.9 \\
\hline MSCC-414-F7 & 3 & 118 & 0.0616 & 0.0576 & 0.0647 & 0.2329 & 1.97 & 10 & 5 & 14.1 \\
\hline MSCC-414-F8 & 3 & 72 & 0.0657 & 0.0603 & 0.0722 & 0.1197 & 1.82 & 7 & 6 & 29.9 \\
\hline MSCC-414-F9 & 4 & 116 & 0.0663 & 0.0594 & 0.0726 & 0.3001 & 2.50 & 5 & 5 & 22.9 \\
\hline MSCC-414-F10 & 4 & 107 & 0.0546 & 0.0518 & 0.0590 & 0.4163 & 1.99 & 6 & 6 & 29.9 \\
\hline MSCC-414-F11 & 5 & 147 & 0.0616 & 0.0589 & 0.0657 & 0.1262 & 1.64 & 14 & 8 & 35.1 \\
\hline
\end{tabular}


Table A.1. continued.

\begin{tabular}{|c|c|c|c|c|c|c|c|c|c|c|}
\hline \multirow{2}{*}{$\begin{array}{l}\text { Fil. } \\
\text { ID } \\
(1)\end{array}$} & \multirow{2}{*}{$\begin{array}{r}N_{\text {sfil }} \\
\text { systems } \\
(2)\end{array}$} & \multirow{2}{*}{$\begin{array}{r}N_{\text {gfil }} \\
\text { gals. } \\
(3) \\
\end{array}$} & \multicolumn{3}{|c|}{ Redshift } & \multirow{2}{*}{$\begin{array}{l}d_{\text {fil }} \\
{\left[\mathrm{h}_{70}^{3} \mathrm{Mpc}^{-3}\right]} \\
(7)\end{array}$} & \multirow{2}{*}{$\begin{array}{c}R_{\mathrm{fil}} \\
{\left[\mathrm{h}_{70}^{-1} \mathrm{Mpc}\right]} \\
\quad(8)\end{array}$} & \multicolumn{2}{|c|}{$N_{\text {nod }}$} & \multirow{2}{*}{$\begin{array}{r}\ell_{\text {fil }} \\
{\left[\mathrm{h}_{70}^{-1} \mathrm{Mpc}\right]} \\
(11)\end{array}$} \\
\hline & & & $\begin{array}{l}\text { [mean, } \\
(4)\end{array}$ & min, & $\begin{array}{c}\max ] \\
(6)\end{array}$ & & & $\begin{array}{r}\text { Filament } \\
(9)\end{array}$ & $\begin{array}{r}\text { Skeleton } \\
(10)\end{array}$ & \\
\hline MSCC-414-F12 & 3 & 14 & 0.0762 & 0.0696 & 0.0801 & 0.1126 & 0.66 & 4 & 4 & 9.8 \\
\hline MSCC-414-F13 & 4 & 66 & 0.0640 & 0.0614 & 0.0674 & 0.1692 & 1.94 & 4 & 4 & 19.2 \\
\hline MSCC-414-F14 & 4 & 93 & 0.0727 & 0.0686 & 0.0758 & 0.4275 & 1.78 & 7 & 6 & 23.6 \\
\hline MSCC-414-F15 & 3 & 31 & 0.0607 & 0.0563 & 0.0640 & 0.1070 & 1.80 & 3 & 3 & 9.0 \\
\hline MSCC-419-F1 & 3 & 130 & 0.1139 & 0.1084 & 0.1210 & 0.1849 & 4.03 & 2 & 2 & 12.8 \\
\hline MSCC-419-F2 & 3 & 88 & 0.1099 & 0.1044 & 0.1182 & 0.1816 & 3.20 & 2 & 2 & 18.1 \\
\hline MSCC-419-F3 & 4 & 36 & 0.1127 & 0.1080 & 0.1191 & 0.0693 & 1.79 & 5 & 4 & 25.2 \\
\hline MSCC-422-F1 & 3 & 11 & 0.1424 & 0.1321 & 0.1528 & 0.0291 & 0.95 & 8 & 6 & 45.0 \\
\hline MSCC-430-F1 & 4 & 151 & 0.0975 & 0.0880 & 0.1066 & 0.1391 & 2.78 & 7 & 4 & 39.2 \\
\hline MSCC-430-F2 & 4 & 23 & 0.0937 & 0.0867 & 0.0999 & 0.1684 & 1.50 & 5 & 4 & 41.1 \\
\hline MSCC-430-F3 & 3 & 12 & 0.0942 & 0.0891 & 0.1015 & 0.0691 & 1.89 & 2 & 2 & 21.2 \\
\hline MSCC-430-F4 & 3 & 104 & 0.1024 & 0.0973 & 0.1041 & 0.0225 & 3.24 & 2 & 2 & 18.3 \\
\hline MSCC-440-F1 & 6 & 184 & 0.1173 & 0.1074 & 0.1284 & 0.1550 & 2.93 & 8 & 6 & 50.2 \\
\hline MSCC-454-F1 & 13 & 687 & 0.0389 & 0.0334 & 0.0466 & 2.7338 & 2.39 & 23 & 13 & 63.5 \\
\hline MSCC-454-F2 & 6 & 498 & 0.0446 & 0.0377 & 0.0500 & 2.3032 & 2.57 & 20 & 9 & 29.1 \\
\hline MSCC-454-F3 & 3 & 146 & 0.0520 & 0.0464 & 0.0557 & 4.0214 & 2.48 & 3 & 3 & 9.3 \\
\hline MSCC-454-F4 & 3 & 146 & 0.0490 & 0.0425 & 0.0551 & 1.5093 & 2.42 & 3 & 3 & 12.8 \\
\hline MSCC-454-F5 & 3 & 39 & 0.0438 & 0.0424 & 0.0460 & 1.1194 & 0.83 & 3 & 3 & 8.6 \\
\hline MSCC-457-F1 & 19 & 908 & 0.0786 & 0.0709 & 0.0877 & 0.4021 & 3.37 & 23 & 11 & 78.4 \\
\hline MSCC-457-F2 & 8 & 256 & 0.0758 & 0.0673 & 0.0830 & 0.1594 & 2.69 & 12 & 8 & 52.5 \\
\hline MSCC-457-F3 & 6 & 179 & 0.0846 & 0.0768 & 0.0896 & 0.2454 & 2.48 & 7 & 5 & 30.6 \\
\hline MSCC-457-F4 & 3 & 92 & 0.0829 & 0.0757 & 0.0881 & 0.1691 & 2.80 & 3 & 3 & 22.2 \\
\hline MSCC-457-F5 & 3 & 77 & 0.0756 & 0.0676 & 0.0785 & 0.0982 & 1.97 & 3 & 3 & 16.8 \\
\hline MSCC-457-F6 & 3 & 13 & 0.0816 & 0.0779 & 0.0872 & 0.0649 & 0.74 & 3 & 3 & 23.3 \\
\hline MSCC-460-F1 & 12 & 536 & 0.1142 & 0.1067 & 0.1242 & 0.1862 & 3.61 & 17 & 9 & 69.3 \\
\hline MSCC-460-F2 & 6 & 214 & 0.1139 & 0.1043 & 0.1237 & 0.1195 & 3.62 & 9 & 6 & 57.9 \\
\hline MSCC-460-F3 & 6 & 82 & 0.1080 & 0.1003 & 0.1159 & 0.0695 & 2.55 & 6 & 5 & 59.7 \\
\hline MSCC-460-F4 & 5 & 63 & 0.1218 & 0.1155 & 0.1304 & 0.0522 & 2.51 & 7 & 5 & 27.6 \\
\hline MSCC-463-F1 & 9 & 489 & 0.0722 & 0.0652 & 0.0788 & 0.3732 & 2.77 & 16 & 10 & 57.4 \\
\hline MSCC-463-F2 & 6 & 352 & 0.0765 & 0.0699 & 0.0839 & 0.3129 & 2.67 & 15 & 10 & 39.0 \\
\hline MSCC-463-F3 & 11 & 313 & 0.0768 & 0.0696 & 0.0842 & 0.2787 & 2.27 & 13 & 11 & 73.6 \\
\hline MSCC-463-F4 & 3 & 234 & 0.0657 & 0.0585 & 0.0743 & 0.4589 & 2.77 & 5 & 4 & 20.8 \\
\hline MSCC-463-F5 & 5 & 192 & 0.0655 & 0.0600 & 0.0730 & 0.4039 & 2.08 & 10 & 8 & 41.9 \\
\hline MSCC-463-F6 & 7 & 233 & 0.0837 & 0.0775 & 0.0894 & 0.2868 & 2.56 & 7 & 6 & 36.9 \\
\hline MSCC-463-F7 & 4 & 8 & 0.0688 & 0.0654 & 0.0739 & 0.0878 & 0.68 & 8 & 7 & 26.8 \\
\hline MSCC-463-F8 & 4 & 119 & 0.0663 & 0.0628 & 0.0698 & 0.4765 & 2.59 & 6 & 4 & 12.5 \\
\hline MSCC-463-F9 & 4 & 99 & 0.0706 & 0.0660 & 0.0764 & 0.2416 & 1.87 & 6 & 5 & 31.9 \\
\hline MSCC-463-F10 & 3 & 83 & 0.0701 & 0.0651 & 0.0754 & 0.3595 & 2.05 & 4 & 3 & 12.7 \\
\hline MSCC-463-F11 & 3 & 106 & 0.0802 & 0.0748 & 0.0861 & 0.1864 & 2.64 & 4 & 4 & 14.8 \\
\hline MSCC-474-F1 & 3 & 881 & 0.0369 & 0.0300 & 0.0444 & 2.7548 & 2.63 & 13 & 5 & 10.5 \\
\hline MSCC-474-F2 & 5 & 407 & 0.0340 & 0.0296 & 0.0387 & 1.3043 & 2.06 & 13 & 7 & 23.4 \\
\hline MSCC-474-F3 & 3 & 147 & 0.0368 & 0.0318 & 0.0412 & 0.2423 & 1.98 & 9 & 8 & 16.5 \\
\hline MSCC-474-F4 & 3 & 176 & 0.0321 & 0.0284 & 0.0388 & 0.7372 & 1.75 & 7 & 5 & 16.5 \\
\hline MSCC-474-F5 & 3 & 114 & 0.0337 & 0.0292 & 0.0372 & 0.3332 & 1.51 & 10 & 6 & 17.2 \\
\hline MSCC-474-F6 & 4 & 193 & 0.0365 & 0.0324 & 0.0423 & 0.5697 & 1.90 & 6 & 6 & 15.3 \\
\hline MSCC-474-F7 & 3 & 330 & 0.0379 & 0.0328 & 0.0406 & 0.1777 & 2.54 & 10 & 7 & 28.3 \\
\hline MSCC-484-F1 & 4 & 109 & 0.1361 & 0.1243 & 0.1501 & 0.0130 & 3.89 & 7 & 6 & 54.6 \\
\hline
\end{tabular}

